University of Tennessee Health Science Center

UTHSC Digital Commons

\title{
T-cell Receptor CDR3 Sequence but Not Recognition Characteristics Distinguish Autoreactive Effector and Foxp3+ Regulatory T-cells
}

Xin Liu

University of Tennessee Health Science Center

Follow this and additional works at: https://dc.uthsc.edu/dissertations

Part of the Medical Cell Biology Commons

\section{Recommended Citation}

Liu, Xin , "T-cell Receptor CDR3 Sequence but Not Recognition Characteristics Distinguish Autoreactive Effector and Foxp3+ Regulatory T-cells" (2010). Theses and Dissertations (ETD). Paper 154. http://dx.doi.org/10.21007/etd.cghs.2010.0188. 


\title{
T-cell Receptor CDR3 Sequence but Not Recognition Characteristics Distinguish Autoreactive Effector and Foxp3+ Regulatory T-cells
}

\author{
Abstract \\ Regulatory T-cells (Treg) play an important role in maintaining immune tolerance to self-antigens and in \\ suppressing excessive immune responses in the host. The source, specificity, and plasticity of the \\ forkhead box transcription factor $3\left(\mathrm{Foxp}^{+}\right)$Treg and conventional T (Tconv) cell populations active at \\ sites of autoimmune pathology are not well characterized. To evaluate this, we combined global \\ repertoire analyses and functional assessments of isolated T-cell receptors (TCR) from TCRa retrogenic \\ mice with autoimmune encephalomyelitis (EAE). Treg and Tconv cell TCR repertoires were distinct, and \\ autoantigen-specific Treg and Tconv cells were enriched in diseased tissue. Autoantigen sensitivity and \\ fine specificity of these cells intersected, implying that differences in responsiveness were not \\ responsible for lineage specification. Notably, autoreactive Treg and Tconv cells could be fully \\ distinguished by an acidic versus aliphatic variation at a single TCR CDR3 residue. Our results imply that \\ ontogenically distinct Treg and Tconv cell repertoires with convergent specificities for autoantigen \\ respond during autoimmunity and argue limited plasticity between Treg and Tconv cells during \\ autoimmune inflammation. \\ Document Type \\ Dissertation \\ Degree Name \\ Doctor of Philosophy (PhD) \\ Program \\ Biomedical Sciences \\ Research Advisor \\ Terrence L. Geiger, M.D., Ph.D. \\ Keywords \\ EAE, Foxp3, Regulatory, T-cell

\section{Subject Categories} \\ Medical Cell Biology | Medical Sciences | Medicine and Health Sciences
}




\title{
T-CELL RECEPTOR CDR3 SEQUENCE BUT NOT RECOGNITION CHARACTERISTICS DISTINGUISH AUTOREACTIVE EFFECTOR AND FOXP3 $^{+}$REGULATORY T-CELLS
}

\author{
A Dissertation \\ Presented for \\ The Graduate Studies Council \\ The University of Tennessee \\ Health Science Center
}

\author{
In Partial Fulfillment \\ Of the Requirements for the Degree \\ Doctor of Philosophy \\ From The University of Tennessee
}

\footnotetext{
By

Xin Liu

May 2010
} 
Chapter 3 C 2009 by American Institute of Biological Sciences All other material (C) 2010 by Xin Liu

All rights reserved 


\section{DEDICATION}

This dissertation is dedicated to my parents,

Mingxian Guan and Mingfang Liu, My husband Ziwei Zhang,

My two adorable boys Victor and Derek, for their love and support 


\section{ACKNOWLEDGEMENTS}

I would like to thank my advisor Dr. Terrence L. Geiger for his guidance, support and help throughout my entire graduate education. His appealing ideas and patient training made this project possible. Dr. Geiger was always there to listen and give advice. He taught me how to think scientifically and present professionally. He showed me the need to be persistent to accomplish any goal and different approaches to resolve a problem. He also taught me how to balance work and family efficiently. I appreciate Dr. Geiger's mentoring not only in science, but also in things beyond science.

I would also like to express my deep gratitude to my other committee members: Dr. Dario Vignali, Dr. David Nelson, Dr. Elizabeth A. Fitzpatrick and Dr. Hongbo Chi, for their guidance, suggestion and support.

I am grateful to all Geiger lab members for their valuable advice, inspiring discussion and friendship. In particular, I thank Meredith A. Steeves and Phuong U. Nguyen for teaching me various techniques, helping my experiments and most importantly, creating a warm, friendly atmosphere that made my graduate experience enjoyable.

I thank my family: my mother Mingxian Guan, for giving me life in the first place, for teaching me how to face difficulties in life when father was long gone, for her unconditional support and encouragement to pursue my interests, even when the interests went beyond boundaries of language, field and geography. I deeply appreciate my husband, Ziwei, for his endless love and support during my entire graduate study. 


\begin{abstract}
Regulatory T-cells (Treg) play an important role in maintaining immune tolerance to self-antigens and in suppressing excessive immune responses in the host. The source, specificity, and plasticity of the forkhead box transcription factor 3 (Foxp3 ${ }^{+}$) Treg and conventional $\mathrm{T}$ (Tconv) cell populations active at sites of autoimmune pathology are not well characterized. To evaluate this, we combined global repertoire analyses and functional assessments of isolated T-cell receptors (TCR) from TCR $\alpha$ retrogenic mice with autoimmune encephalomyelitis (EAE). Treg and Tconv cell TCR repertoires were distinct, and autoantigen-specific Treg and Tconv cells were enriched in diseased tissue. Autoantigen sensitivity and fine specificity of these cells intersected, implying that differences in responsiveness were not responsible for lineage specification. Notably, autoreactive Treg and Tconv cells could be fully distinguished by an acidic versus aliphatic variation at a single TCR CDR3 residue. Our results imply that ontogenically distinct Treg and Tconv cell repertoires with convergent specificities for autoantigen respond during autoimmunity and argue limited plasticity between Treg and Tconv cells during autoimmune inflammation.
\end{abstract}




\section{TABLE OF CONTENTS}

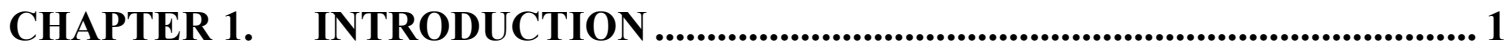

1.1 An Introduction to Experimental Allergic Encephalomyelitis (EAE) ..................... 1

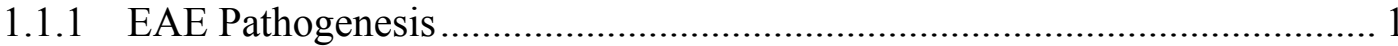

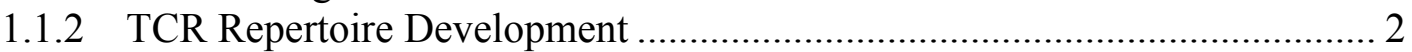

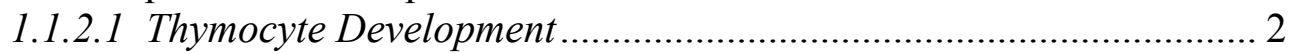

1.1.2.2 TCR Repertoire Diversity ......................................................... 3

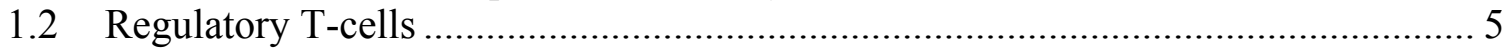

1.2.1 Suppressor Mechanisms Utilized by Regulatory T-cells.............................. 7

1.2.1.1 Role of Cytolysis .......................................................................... 7

1.2.1.2 Role of Immunosuppressive Cytokines ............................................ 8

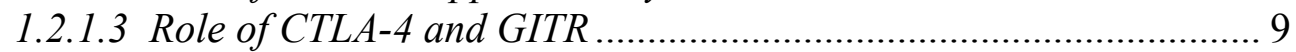

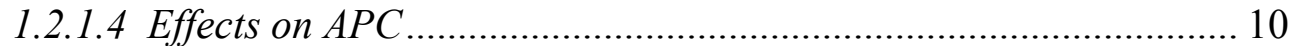

1.2.2 Thymic and Extrathymic Origin of Foxp3 $3^{+}$Treg ...................................... 10

1.2.2.1 Thymic Development of Foxp $3^{+}$Treg Cells ................................. 10

1.2.2.2 Peripheral Generation of Foxp $3^{+}$Treg Cells............................... 11

1.2.3 Specificity of Regulatory T-cells ............................................................... 13

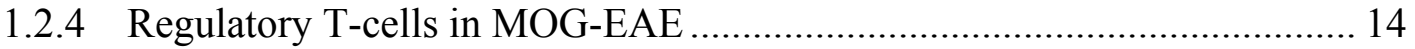

1.2.5 Repertoire Analysis of Treg Cells....................................................... 15

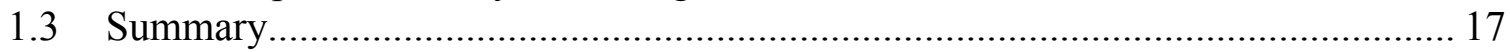

CHAPTER 2. MATERIALS AND METHODS ................................................. 18

2.1 Methods of TCR Repertoire Analysis …………............................................ 18

2.1.1 Vector Construction for TCR $\alpha$................................................................ 18

2.1.2 Generation of 1MOG244.2TCR $\alpha$ Retrogenic Mice ..................................... 18

2.1.2.1 Retroviral Production and Cellular Transduction ......................... 18

2.1.2.2 Retroviral Transduction and Transplant of Bone Marrow Cells ... 18

2.1.3 EAE Induction and Clinical Evaluation.................................................. 20

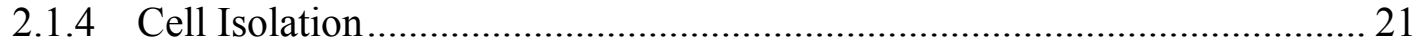

2.1.4.1 Preparation of the Splenocyte Suspension ……………………...... 21

2.1.4.2 Preparation of the Thymocytes and Lymphocytes Cell

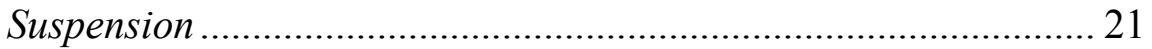

2.1.4.3 Isolation of CNS-infiltrating T-lymphocytes................................... 21

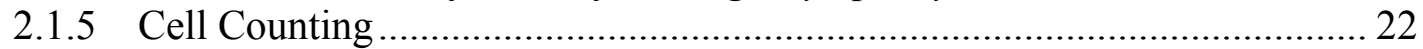

2.1.6 Flow Cytometry ……………………………….................................. 22

2.1.6.1 Flow Cytometry (FACS) Analysis of 1MOG244.2 Retrogenic

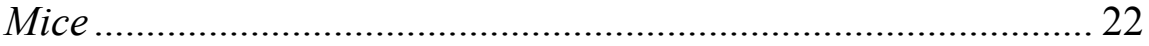

2.1.6.2 VB Staining of 1MOG244.2 Retrogenic Mice................................ 23

2.1.6.3 Cell Separation by Flow Cytometry ............................................... 23

2.1.7 RNA Isolation and cDNA Transcription …………................................... 23

2.1.8 Polymerase Chain Reaction (PCR) Amplification ....................................... 23

2.1.9 Agarose Gel Electrophoresis and PCR Product Purification....................... 24 


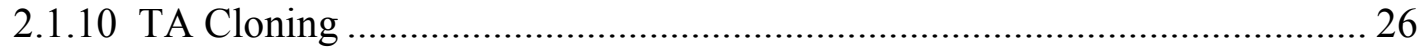

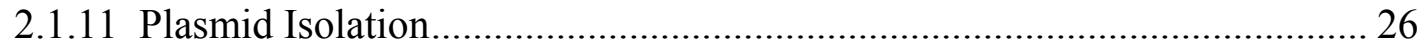

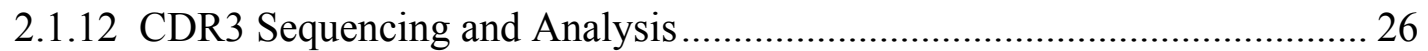

2.2 MOG-specificity of Transduced 4G4 Hybridomas ................................................ 26

2.2.1 Vector Construction ............................................................................. 26

2.2.2 Transformation of CD4-4G4 T-cell Hybridoma........................................ 28

2.2.3 Cytokine (IL-2) Analysis by Enzyme-linked Immunosorbent Assay

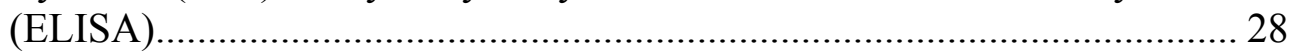

2.3 Generation of TCR-retrogenic Mixed Chimeras .................................................. 29

2.4 Flow Cytometric Analysis of TCR-retrogenic Mixed Chimeras ………………..... 29

CHAPTER 3. GENERATION OF 1MOG244.2TCR $\alpha$ RETROGENIC MICE .. 30

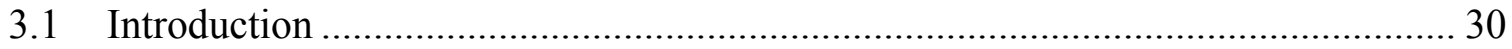

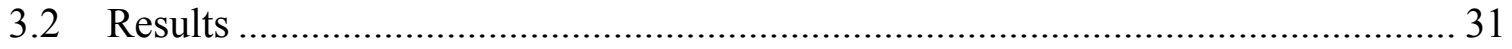

3.2.1 Retrogenic Mice Expressing a Single TCR V $\alpha$ Chain ................................ 31

3.2.2 Phenotype of 1MOG244.2TCR $\alpha$ Retrogenic Mice........................................ 31

3.2.3 V $\beta$ Use in 1MOG244.2TCR $\alpha$ Splenic T-lymphocytes ................................ 34

3.2.4 Increased TCR V $\beta 8.2$ Use in CNS-infiltrating 1MOG244.2 $\alpha$ T-cells ........ 34

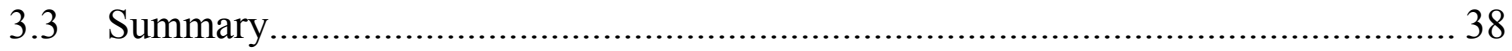

CHAPTER 4. TCR $\beta$ REPERTOIRE ANALYSIS OF 1MOG244.2TCR $\alpha$ RETROGENIC MICE ....................................................................... 39

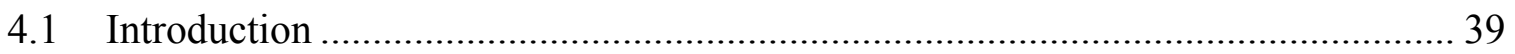

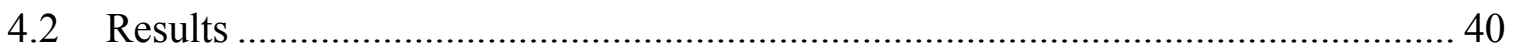

4.2.1 TCR J $\beta$ and CDR3 Use in MOG-EAE ..................................................... 40

4.2.2 Limited Overlap of Treg and Tconv Repertoires.......................................... 44

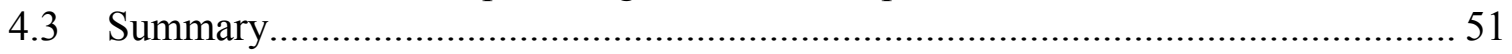

CHAPTER 5. ANALYSIS OF TCR IN 1MOG244.2TCR $\alpha$ RETROGENIC MICE............................................................................................. 53

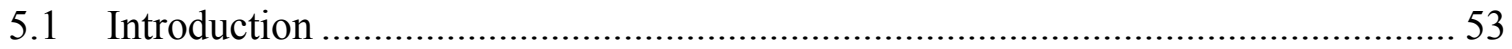

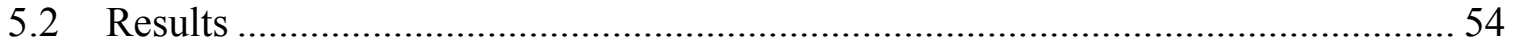

5.2.1 MOG-specificity among Treg and Tconv TCR ......................................... 54

5.2.2 Segregation of MOG-Specific Treg and Tconv Cell TCR Based on

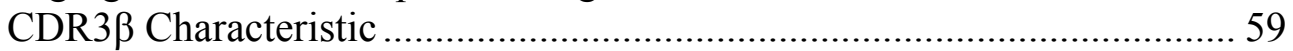

5.2.3 Sensitivity and Fine Specificity of MOG-specific TCR for Auto-antigen .. 59

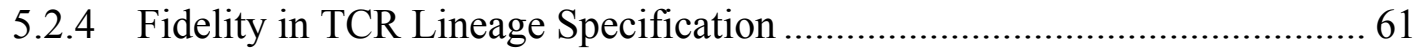

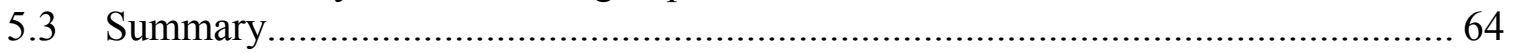




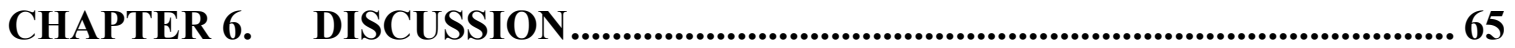

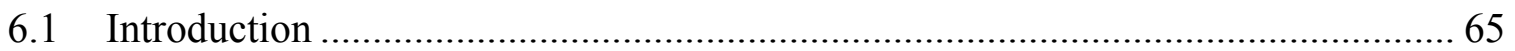

6.2 Developing a Retrogenic Model of EAE to Facilitate Repertoire Analysis............ 65

6.2.1 Why Does the TCR $\alpha$ Chain Need to be Fixed?.......................................... 65

6.2.2 Does a Fixed $\alpha$ Chain Influence the Course of MOG-EAE

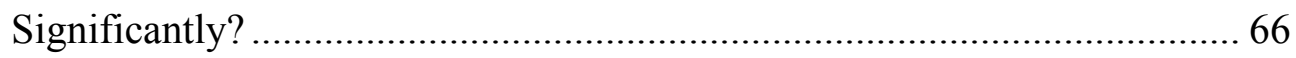

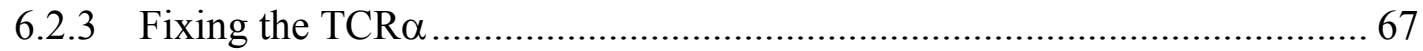

6.3 Interconversion and Origins of Treg and Tconv Cells in Autoimmunity ............... 68

6.3.1 Limitations of the TCR Repertoire Analysis …………………………...... 71

6.3.2 Future Studies ..................................................................................... 71

6.4 Segregation of Tconv and Treg Cells Based on CDR3 $\beta$ Sequence ........................ 72

6.5 Future Plans to Confirm the Segregation of Treg and Tconv Based on TCR

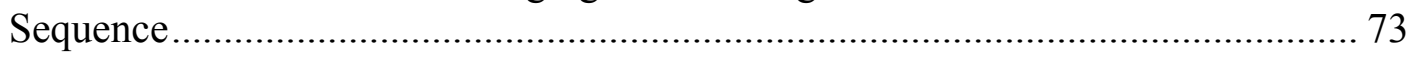

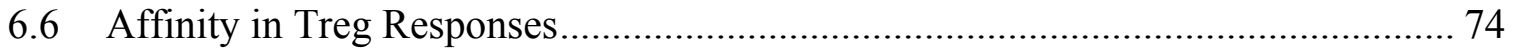

6.6.1 Models of Treg Repertoire Selection.................................................... 74

6.6.2 Sensitivity and Fine Specificity of MOG-specific Treg and Tconv TCR ... 74

6.7 Antigen Specificity in Regulatory T-cell Responses............................................... 76

6.7.1 Does MOG-specific T-cell Cross-react with Alternative Self-antigen Which Mediates Thymic Selection? ……………………............................ 76

6.7.2 What Do Non-MOG-specific Treg TCR Recognize?.................................... 77

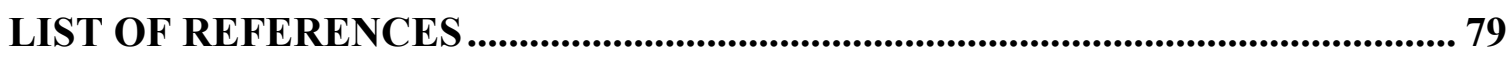

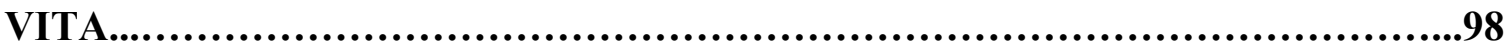




\section{LIST OF TABLES}

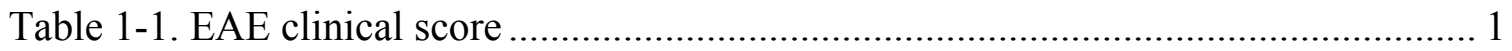

Table 2-1. PCR program used to amplify CDR3 region........................................... 24

Table 4-1. Isolation of TCR V $\beta$ sequences from retrogenic mice................................. 43

Table 5-1. Features of CDR3 $\beta$ used to reconstitute full-length TCR cDNA.................... 55 


\section{LIST OF FIGURES}

Figure 1-1. Signaling cascades stimulated by the T-cell receptor.

Figure 1-2. Generation of T-cell receptor diversity by somatic recombination of TCR gene segments

Figure 2-1. Structure of retroviral constructs............................................................... 19

Figure 2-2. PCR amplification of CDR3 region........................................................ 25

Figure 2-3. Structure of modified retroviral constructs containing new BamHI and XhoI sites.

Figure 3-1. EAE induction in retrogenic mice.

Figure 3-2. Thymocyte reconstitution in retrogenic mice 33

Figure 3-3. Lymphoid reconstitution of retrogenic mice 35

Figure 3-4. V $\beta$ use by splenic Tconv in $1 \mathrm{MOG} 244.2 \alpha$ and $\mathrm{C} 57 \mathrm{BL} / 6$ mice...... 36

Figure 3-5. V $\beta$ use by retrogenic Treg and Tconv in pre-immune splenocytes and splenocytes and CNS-infiltrating cells from mice with EAE

Figure 4-1. J $\beta$ used in Tconv and Treg cells.

Figure 4-2. Population differences among TCR subsets in mice with EAE...................... 45

Figure 4-3. Distribution of shared CDR3 sequences.

Figure 4-4. Frequency representation of the most common splenic $V \beta 8.2^{+} \mathrm{J} \beta 2.7^{+}$ $\mathrm{CDR} 3$ isolates.

Figure 4-5. Frequency representation of the most common CNS-derived $\mathrm{V} \beta 8.2^{+} \mathrm{J} \beta 2.7^{+} \mathrm{CDR} 3$ isolates. 50

Figure 4-6. Length, charge, and hydrophilicity of isolated CDR3.. 52

Figure 5-1. Expression of TCR on CD4.4G4 cells.. 57 
Figure 5-2. MOG-specificity among isolated TCR.

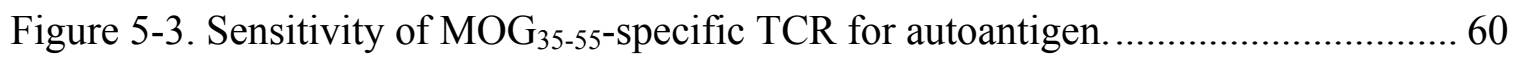

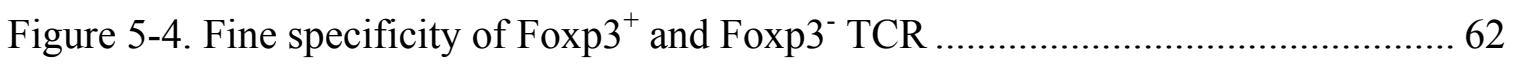

Figure 5-5. Foxp3 expression among $\mathrm{CD} 4^{+} \mathrm{T}$-cells in TCR-retrogenic mixed chimeras.

Figure 6-1. Models of interconversion between Tconv and Treg cells in MOG-EAE.... 70

Figure 6-2. Generation of specificities of the Treg repertoire. 75 


\section{CHAPTER 1. INTRODUCTION}

\subsection{An Introduction to Experimental Allergic Encephalomyelitis (EAE)}

Experimental allergic encephalomyelitis (EAE) is a demyelinating disease of the central nervous systems. For over half a century, it has been used as an animal model of human MS. EAE can be induced in different species including mice, rats, guinea pigs, rabbits, macaques, rhesus monkeys and marmosets, among which mice and rats are the most commonly used species due to their low cost, ready availability, lifespan and large number of genetically modified strains. EAE can be an acute, chronic, or relapsingremitting type disease depending on the induction method and types of animal. For example, C57BL/6 mice develop EAE 8-14 days after immunization and may remain paralyzed for a prolonged period (Mendel et al., 1995). In contrast, SJL mice resemble the remitting-replapsing form of MS, with alternating episodes of paralysis and recovery (Whitham et al., 1991).

EAE is most commonly induced in mice by immunization with CNS-derived auto-antigens in complete Freund's adjuvant (CFA). Myelin oligodendrocyte glycoprotein $\left(\mathrm{MOG}_{35-55}\right)$, myelin proteolipid protein $\left(\mathrm{PLP}_{139-151}\right)$ and myelin basic protein $\left(\mathrm{MBP}_{1-9}\right)$ are immunodominant epitopes in the $\mathrm{C} 57 \mathrm{BL} / 6$, SJL and B10.PL mice strains respectively (Zamvil and Steinman, 1990). Alternatively, passive transfer of restimulated T-cells that recognize myelin autoantigens can also induce EAE (Yoshizawa et al., 1998; Zamvil et al., 1985). Disease progression can be monitored by clinical observation, as an ascending paralysis or paresis develops in affected animals. The following scoring system is most commonly applied (Table 1-1).

\subsubsection{EAE Pathogenesis}

In the healthy individual, lymphocytes traffic into the central nervous system (CNS) is tightly restricted by the blood-brain barrier (BBB). After immunization with

Table 1-1. EAE clinical score.

\begin{tabular}{ll}
\hline Score & Clinical Symptoms \\
\hline 0 & Healthy \\
1 & Limp tail, loss of tail tone \\
2 & Limp tail and hind limb paraparesis \\
3 & Complete hind limb paralysis \\
4 & Complete hind limb and partial front limb paralysis \\
5 & Morbid \\
\hline
\end{tabular}


$\mathrm{MOG}_{35-55}$ in CFA, circulating lymphocytes and monocytes traffic across the BBB. Tcells are restimulated by myelin presented by dendritic cells in the CNS, resulting in inflammation and demyelination. The recruitment process is mediated by the sequential interaction of different adhesion molecules on lymphocytes and the inflamed BBB.

During the course of EAE, CNS endothelium upregulates expression of intercellular adhesion molecule (ICAMT)-1 and vascular cell adhesion molecule (VCAM)-1 (Engelhardt, 2006; Steffen et al., 1994). Activated myelin-specific T-cells upregulate adhesion molecules and chemokine receptors, allowing them to adhere and transmigrate through the endothelium (Alt et al., 2002). Blocking antibodies against VCAM-1 or its ligand, $\alpha 4$-integrins, inhibits the development of EAE and inflammatory T-cell accumulation in the CNS, implying that the recognition of VCAM-1/ $\alpha 4$-integrins mediates the adhesion of T-cells to inflamed CNS vessels (Engelhardt et al., 1998). Lymphoid chemokines CCL19 and CCL21 are also found to be expressed in BBB endothelium and CNS venules during EAE (Alt et al., 2002). Their common receptor CCR7 is detected on encephalitogenic T-cells. Functional ablation of CCR7 or blocking CCL19 and CCL21 reduces the accumulation of T-cells to inflamed venules in the CNS, suggesting that CCL19 and CCL21 are involved in the recruitment of encephalitogenic Tcells. Besides, the b2-integrin LFA-1 expressed on encephalitogenic T-cells was required for transendothelial migration by engaging with endothelial ICAM-1 and/or ICAM-2 (Laschinger et al., 2002; Lyck et al., 2003).

The key histopathologic feature of EAE is the destruction of the axonal myelin shealth in the CNS. This histopathology mimics that seen in MS, with T-cell and macrophage dominated inflammation, extensive demyelination, axonal and neuronal damage. $\mathrm{CD}^{+}{ }^{\mathrm{T}}$-cells, $\mathrm{CD} 8^{+} \mathrm{T}$-cells, B-cells and activated microglia/macrophages are responsible for the lesion development in patients with MS. One difference in most, but not all mouse systems, is an increased reliance on $\mathrm{CD}^{+} \mathrm{T}$-cells and macrophages, with smaller roles for $\mathrm{CD} 8^{+} \mathrm{T}$-cells and $\mathrm{B}$ lymphocytes, though in some cases polymorphs are also detected in acute EAE lesions (Sobel and Kuchroo, 1992). Most myelin-reactive Tcells are deleted during thymic development because many myelin antigens were expressed in the thymus. However, the deletion cannot be complete, and auto-reactive Tcells escaping negative selection constitute the peripheral T-cell repertoire and must be kept in check by peripheral tolerance. In EAE, auto-reactive T-cells have to be activated by local antigen presenting cells (APCs) and appropriate costimulatory molecules (Chang et al., 1999), then migrate across the BBB into the CNS to initiate inflammation and tissue injury.

\subsubsection{TCR Repertoire Development}

\subsubsection{Thymocyte Development}

The T-cell receptor (TCR), expressed on the surface of T-lymphocytes, is responsible for antigen recognition. It is a heterodimer composed of two distinct chains, 
$\alpha$ and $\beta$ chain in $95 \%$ of T-cells, and $\gamma$ and $\delta$ chains in 5\% of T-cells (Davis et al., 1998). The $\alpha \beta$ TCR requires coexpression of invariant CD3 polypeptides, a collection of protein dimers $(\gamma \varepsilon, \delta \varepsilon$ and $\zeta \zeta)$. That associate with the $\alpha \beta$ or $\gamma \delta$ chains. Neither CD3 nor $\alpha \beta$ dimer are expressed on the cell surface separately, and the partner proteins are required to prevent the isolated protein from degradation (Manolios et al., 1991). The Ig-like $\alpha \beta$ heterodimer is responsible for antigen recognition. Transfection of cDNAs encoding $\alpha$ and $\beta$ chains from one T-cell clone into another results in the transfer of reactivity to both peptide antigen and MHC (Dembic et al., 1986). Antibodies directly stimulating the CD3 molecules elicit the same T-cell activation as stimulation with pMHC. The CD3 molecules initiate signal transduction upon antigen recognition. This process relies on the cytoplasmic domains of the CD3 molecules which contain immunoreceptor tyrosinebased activated motifs (ITAMs). Activation of T-cells leads to phosphorylation of these residues and their association with protein tyrosine kinases (PTKs), such as tyrosineprotein kinase (fyn), leukocyte-specific protein tyrosine kinase (lck), and zeta-chain associated protein kinase (ZAP-70), that further propagate the signal into the cell (Figure $1-1)$.

Similar to immnologlobulin heavy and light chains, all TCR $\alpha$ polypeptides have distinct variable $(\mathrm{V})$ and joining $(\mathrm{J})$ regions. In addition to $\mathrm{V}$ and $\mathrm{J}$ segment, the TCR $\beta$ contains diversity (D) segments. During the T-cell development in the thymus, $\beta$-chain genes undergo rearrangement. First, a $D \beta$ locus rearranges to a $J \beta$ locus, then these associate with a V $\beta$. If the rearrangement produces an in frame TCR $\beta$, the functional $\beta$ chain will pair with a surrogate $\alpha$ chain, Pre-T-cell $\alpha(\mathrm{pT} \alpha)$, and is expressed on the cell surface along with the $\mathrm{CD} 3$ molecules. Failure to successfully rearrange a $\beta$ chain leads to clonal deletion. The assembly of the CD3: pT $\alpha$ leads to cell proliferation, the arrest of further $\beta$ chain rearrangement and the expression of CD8 and CD4. In the double positive thymocytes, TCR $\beta$ rearrangement must be followed by a successful TCR $\alpha$ gene rearrangement within approximately 3 days after the proliferative burst. Otherwise, cells die of "neglect". The double positive thymocytes express a low level of T-cell receptors and must pass a second TCR dependent checkpoint - positive selection. The criteria here are more stringent than for $\beta$ selection. They require both a translatable protein coding sequence and recognition of self MHC molecules. If the TCR cannot bind to self MHC molecules, it will be neglected and die. Cells passing positive selection express high levels of the TCR, and undergo negative selection, in which self reactive cells are eliminated. These critical developmental checkpoints yield a TCR repertoire that can respond to a variety of foreign antigens, but minimizes self-reactivity. Only $5 \%$ of the double positive cells satisfactorily pass the checkpoints and mature into single positive Tcells that exit the thymus and populate the periphery.

\subsubsection{TCR Repertoire Diversity}

TCR repertoire diversity results from combinatorial diversity and junctional diversity. Combinatorial diversity is generated by somatic recombination from multiple germline gene segments. The mouse TCR $\alpha$ locus has $\sim 100 \mathrm{~V} \alpha$ (TRAV) gene, and $\sim 50 \mathrm{~J} \alpha$ 


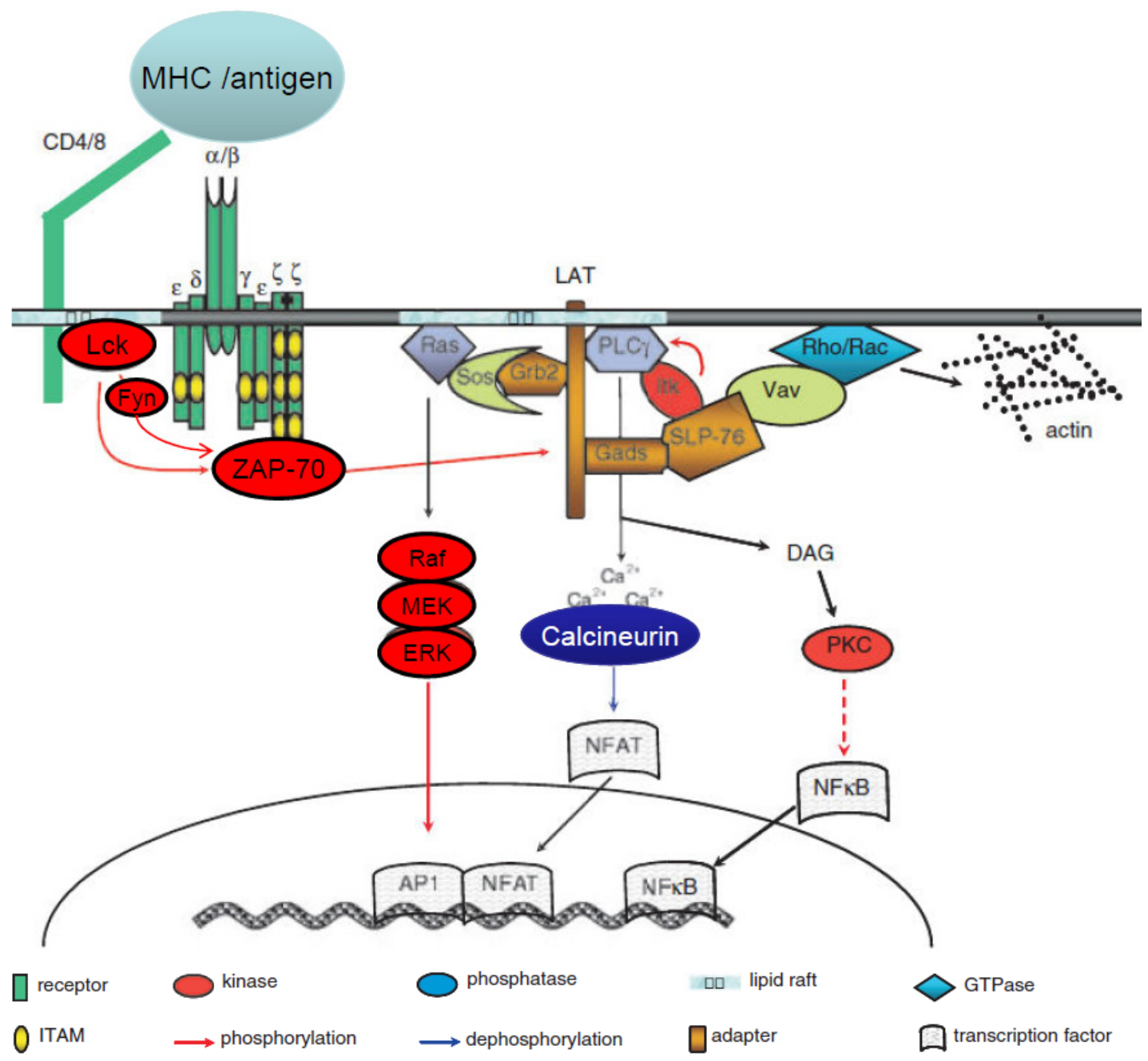

Figure 1-1. Signaling cascades stimulated by the T-cell receptor.

Adapted with permission. Razzaq, T.M., Ozegbe, P., Jury, E.C., Sembi, P., Blackwell, N.M., and Kabouridis, P.S. (2004). Regulation of T-cell receptor signalling by membrane microdomains. Immunology 113, 413-426. 
(TRAJ) gene segments. The TCR $\beta$ locus has $25 \mathrm{~V} \beta$ (TRBV), $2 \mathrm{D} \beta$ (TRBD) and $12 \mathrm{~J} \beta$ (TRBJ) gene segments. The maximum number of possible combinations of these gene segments is the product of the numbers of $\mathrm{V}, \mathrm{J}$ and $\mathrm{D}$ segments in each locus. The diversity implied therein would be much less than the actual diversity of antigen receptors in mature lymphocytes. In fact, a large contribution to the TCR diversity is made by the removal or addition of nucleotides between $\mathrm{V}$ and $\mathrm{D}, \mathrm{D}$ and $\mathrm{J}$, or $\mathrm{V}$ and $\mathrm{J}$ segments at the time of joining, namely, junctional diversity. Nucleotides (P sequences) may be added to broken DNA ends to repair asymmetric RAG-mediated cleavage. Other nucleotides ( $\mathrm{N}$ regions) may be added to the sites of $\mathrm{VD}, \mathrm{VJ}$, or $\mathrm{DJ}$ junctions by the action of the terminal deoxyribonucleotidyl transferase (TdT), generating new sequences that are not present in the germline (Figure 1-2). In addition, random paring of $\alpha$ and $\beta$ chains introduces additional diversity. Taking all these factors together, a theoretical maximum diversity is $10^{15}$ unique TCR. In the thymus, $95 \%$ of the precursor cells are eliminated through both positive and negative selection. This still leaves more than $10^{13}$ possible TCRs that can be produced. The actual diversity of the naive mouse spleen Tcell repertoire is estimated to be $\sim 2 \times 10^{6}$ using the immunoscope technique, suggesting the average size of a clone expressing a single TCR is $\sim 10$ cells in spleen (Casrouge et al., 2000).

TCR diversity is localized to complementarity-determining regions (CDRs) on both chains. CDR1 and 2 regions, encoded by the $\mathrm{V}$ gene segment, are responsible for interacting with MHC molecules; whereas the CDR3 is the most variable part of the TCR. It is encoded by the juxtaposition of rearranged V-(D)-J or V-J segments. CDR3 forms a flexible loop positioned to associate with the peptide-MHC complexes. Characterization of CDR3 sequence variation therefore provides a good measure of TCR diversity in an antigen selected repertoire.

Because many more TCRs may form than the number of T-cell clones that enter the circulation, there are huge differences between repertoires among individuals. In most T-cell responses, the TCR repertoires consist of TCRs with private specificity, that is TCR only observed within one individual. In addition public or shared TCRs among multiple individuals may be seen. Public TCRs are observed in both acute and persistent pathogen infection and autoimmune diseases such as EAE. Why they exist is unclear. A potential explanation is certain bias promotes selection of certain TCR. That includes unique structural features of TCR or the pMHC complex, biased TdT activity and convergent recombination, and selective expansion in periphery.

\subsection{Regulatory T-cells}

T-cells are crucial to adaptive immunity and function both as effector cells and helper cells in humoral and cell mediated responses. The activated immune system also requires homeostatic control mechanisms to down-regulate over-exuberant immune responses. Tolerance to self-antigens includes a central and a peripheral component. Central tolerance refers to negative selection of auto-reactive T-cells in thymus. Peripheral tolerance consists of T-cell deletion, T-cell anergy and regulatory T-cell (Treg) 

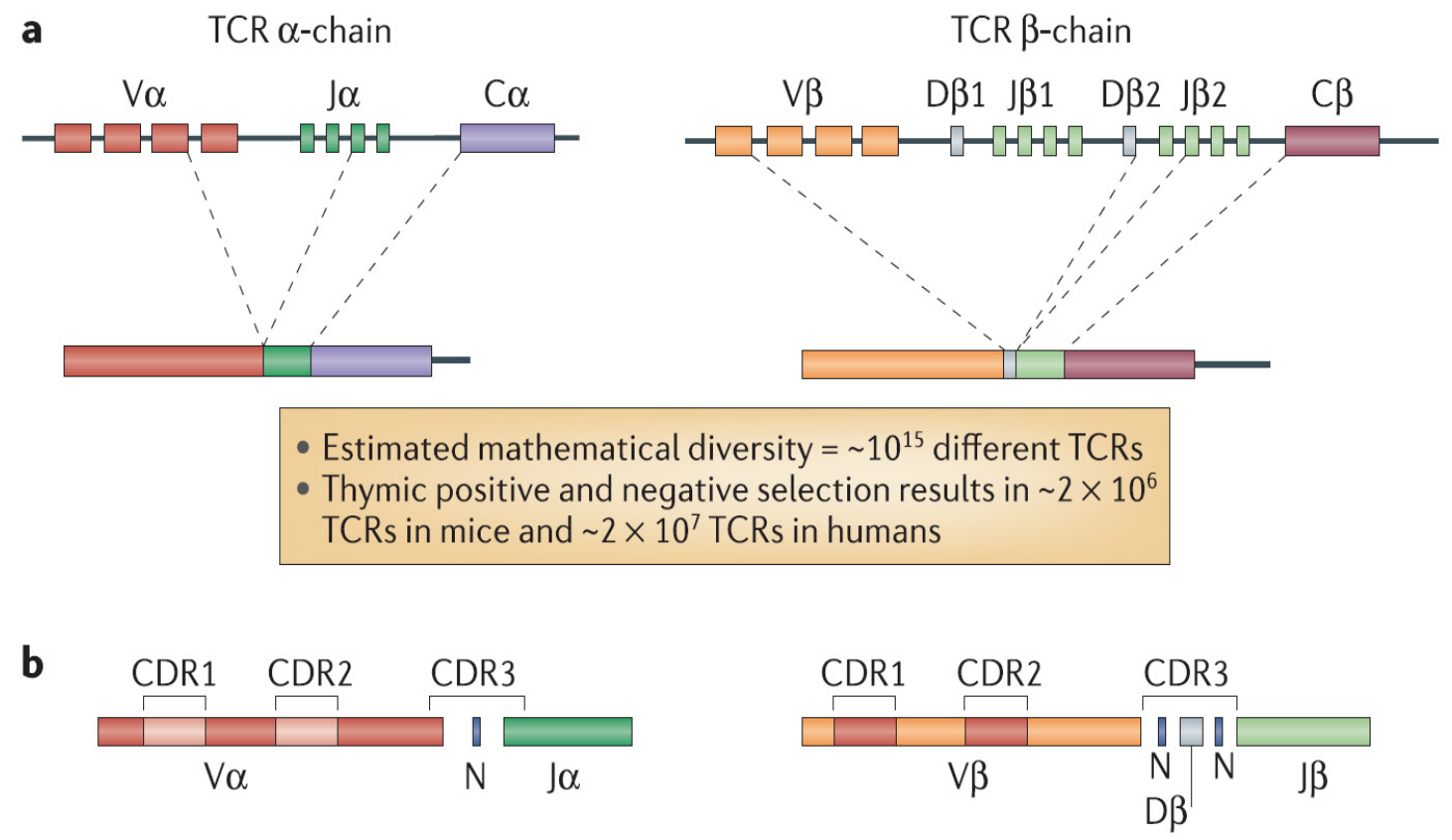

Figure 1-2. Generation of T-cell receptor diversity by somatic recombination of TCR gene segments.

Reprinted with permission. Turner, S.J., Doherty, P.C., McCluskey, J., and Rossjohn, J. (2006). Structural determinants of T-cell receptor bias in immunity. Nat Rev Immunol 6, 883-894. 
suppression in the periphery (Miller and Heath, 1993; Schwartz, 1990). In mouse neonatal thymectomy (nTx) studies, nTx on the third day of life (d3Tx), but not d1Tx or d7Tx, developed on autoimmune disease that could be prevented by thymus transplant or $\mathrm{CD}^{+}$T-cell reconstitution from normal adult mice (Nishizuka and Sakakura, 1969). This suggested that autoreactive T-cells were exported from the thymus earlier than the suppressor cells, and that these cells were also $\mathrm{CD} 4^{+} \mathrm{T}$-cells.

Sakaguchi et al. first proposed that a subset of $\mathrm{CD}^{+} \mathrm{T}$-cells, that constitutively express the CD25 antigen (IL-2R $\alpha$ chain), function as regulatory T-cells in the normal mouse (Asano et al., 1996; Sakaguchi et al., 1995; Suri-Payer et al., 1998). Depletion of $\mathrm{CD} 4{ }^{+} \mathrm{CD} 25^{+} \mathrm{T}$-cells leads to organ specific autoimmune disease in mice, and adoptive transfer of the $\mathrm{CD} 25^{+}$cells prevent autoimmunity. $\mathrm{CD} 4{ }^{+} \mathrm{CD} 25^{+} \mathrm{T}$-cells represent $10 \%$ $15 \%$ of mouse $\mathrm{CD} 4^{+}$lymphocytes, and $5 \%-10 \%$ of $\mathrm{CD} 4^{+}$SP thymocytes. Compared with $\mathrm{CD} 4^{+} \mathrm{CD} 25^{-} \mathrm{T}$-cells, $\mathrm{CD} 4^{+} \mathrm{CD} 25^{+} \mathrm{T}$-cells express slightly higher levels of CD5, cytotoxic T-lymphocyte antigen (CTLA-4) and glucocorticoid-induced TNF-like receptor (GITR), lymphocyte-activation gene (LAG-3), 4-1BB, CD28, CD134, CD80/86 (B7) as well as intermediate and low levels of CD45RB. However, none of those is restricted to Treg cells (Ndhlovu et al., 2004; Paust et al., 2004; Workman et al., 2009). One unique feature of Treg cells is that they express the $\mathrm{X}$ chromosome-encoded forkhead transcription factor 3 (Foxp3), which serves as a key player in the biology of CD $4{ }^{+} \mathrm{CD} 25^{+}$ Treg cells (Brunkow et al., 2001; Fontenot et al., 2003; Hori et al., 2003; Khattri et al., 2003). Expression of Foxp3 is largely restricted to Treg cells and requires Treg cell differentiation (Fontenot et al., 2003; Fontenot et al., 2005b; Hori et al., 2003; Wan and Flavell, 2007). IPEX syndromes (immune dysregulation, polyendocrinopathy, enteropathy, X-linked) in humans and scurfy mice have mutations in the Foxp3 gene. Foxp3 deficient mice show defects in Treg development, and retroviral transduction of Foxp3 into naive CD25- T-cells led to the acquisition of suppressor activity both in vitro and in vivo (Fontenot et al., 2005b).

\subsubsection{Suppressor Mechanisms Utilized by Regulatory T-cells}

Defining the mechanisms of Treg suppressive function is crucial in understanding of peripheral tolerance. It has been shown that Treg cells utilize multiple means to limit immune responses, such as suppression by cell contact-dependent cytolysis on responder T-cells or APCs, suppression by inhibitory cytokines or deprivation of cytokines, suppression by surface molecules (CTLA-4 or GITR), and suppression by modulation of dendritic cell maturation (Vignali et al., 2008).

\subsubsection{Role of Cytolysis}

An early study has shown that $\mathrm{CD} 25^{+} \mathrm{T}$-cells were unable to suppress the in vitro proliferation of responder $\mathrm{CD} 4{ }^{+} \mathrm{CD} 25^{-} \mathrm{T}$-cells when the two populations were separated by a semi-permeable membrane. This suggests that suppression requires cell contact or close proximity between Treg cells and their targets (Thornton and Shevach, 1998). 
Human activated $\mathrm{CD} 4^{+} \mathrm{CD} 25^{+}$Foxp $3^{+}$Treg cells express granzyme A and kill activated $\mathrm{CD}^{+}$and $\mathrm{CD}^{+}{ }^{+}$-cells in a perforin-dependent, Fas-FasL-independent manner.

Activation of mouse Treg cells also results in up-regulation of granzyme B expression (Gondek et al., 2005; Grossman et al., 2004). However, another study demonstrated that highly activated Treg cells could kill APCs but not bystander B-cells or T-cells in a cellcontact-dependent manner (Zhao et al., 2006). Besides granzyme A and B, a recent study showed that galectin-1, which can induce T-cell apoptosis, was up-regulated in human and mouse Treg cells, and galectin-1 deficient Treg cells have reduced suppressive function in vitro (Garin et al., 2007). Nevertheless, these studies suggest more work is needed to determine whether Treg cells can develop into cytotoxic cells in vivo and the cytolytic mechanisms.

\subsubsection{Role of Immunosuppressive Cytokines}

In vivo studies have shown that the inhibitory cytokines such as IL-10, transforming growth factor (TGF- $\beta$ ) are required for Treg mediated suppression in some disease models. In allergy and asthma models, Treg cells control disease in a manner partially dependent on IL-10 (Hawrylowicz and O'Garra, 2005) or on both IL-10 and TGF- $\beta$ (Joetham et al., 2007). In colitis studies, administration of a blocking anti-IL-10 receptor $\mathrm{mAb}$ abrogated suppression of colitis by $\mathrm{CD}^{+} \mathrm{CD} 45 \mathrm{RB}^{\text {low }} \mathrm{T}$-cells, and Treg cells from IL-10 deficient mice failed to suppress colitis, although they suppress autoimmune gastritis (AIG) (Asseman et al., 1999). In EAE model, Treg cells have been shown to produce IL-10 in CNS during recovery phase (Kohm et al., 2002; Korn et al., 2007; McGeachy et al., 2005). One study showed that Treg cells from IL-10 deficient mice were unable to suppress active EAE (Zhang et al., 2004). However, the Treg cellspecific ablation of IL-10 showed no spontaneous systemic autoimmunity, but result in enhanced pathology in the mucosal surface of the intestine and the lung (Rubtsov et al., 2008). Production of TGF- $\beta$ by Treg cells is also shown indispensable for suppression of colitis (Fahlen et al., 2005; Zwar et al., 2006). However, other studies have failed to identify the role for TGF- $\beta$ in Treg-mediated suppression in IBD. In this study, Treg cells from neonatal TGF- $\beta 1$ deficient mice can still suppress the incidence and severity of IBD (Kullberg et al., 2005). Taken together, these findings suggest that the importance of IL-10 and TGF- $\beta$ in Treg mediated suppression is dependent on the environment and experimental setting.

In contrast to the in vivo studies, there is little evidence that suppressive cytokines play a role in Treg-mediated suppression in vitro. For example, the addition of neutralizing anti-IL-10 or anti-IL-4 antibodies to in vitro cultures failed to abrogate suppressive effects of Treg cells. And CD $4^{+} \mathrm{CD} 25^{+} \mathrm{T}$-cells purified from mice deficient in IL-4 or IL-10 showed comparable suppression to wild-type Treg (Thornton and Shevach, 1998). The role of TGF- $\beta$ in suppression is ambiguous. One study showed that inhibition of in vitro suppression was observed in the presence of high concentrations of a neutralizing anti-TGF- $\beta$ antibody, and the author proposed that Treg cells exert suppression by a cell-cell interaction involving cell surface TGF- $\beta 1$ (Nakamura et al., 2001). However, subsequent analyses have shown that responder T-cells from dominant- 
negative TGF- $\beta$ receptor mice were fully susceptible to suppression by Treg cells in vitro. Furthermore, Treg cells from neonatal TGF- $\beta 1$ deficient mice were as suppressive as Treg from wild-type mice, confirming TGF- $\beta$ is not critical to the suppression by Treg cells in vitro (Piccirillo et al., 2002). Studies demonstrated that the suppressor function of activated Treg cells in vitro is antigen nonspecific. When Treg cells from TCR transgenic mice were activated and expanded in vitro, the activated suppressors were able to inhibit fresh $\mathrm{CD} 4^{+} \mathrm{CD} 25^{-} \mathrm{T}$-cells specific for different antigens (Thornton and Shevach, 2000). Collectively, these studies demonstrated that the suppressive effects of Treg cells in vitro are less likely cytokine dependent and antigen non-specific.

Recently, a novel inhibitory cytokine, IL-35, has been described that is specifically produced by Treg and is required for maximal regulatory activity (Collison et al., 2007). Ectopic expression of IL-35 confers regulatory activity on naïve T-cells and recombinant IL-35 suppresses T-cell proliferation in vitro. A later study showed Treg cells expressed substantial IL-35 following contact with Tconv cells, and this potent suppression was in a cell contact, IL-35 and IL-10 dependent manner (Collison et al., 2009). Although the identification of IL-35 brings new information to Treg cell portfolio, there is much more that remains to be defined, such as its contribution to Treg function in vivo, or whether IL-35 plays a role in the development and function of DCs and macrophages.

IL-2 was identified as a T-cell growth factor many years ago (Schimpl and Wecker, 1972). Treg cells do not produce IL-2, but express high affinity IL-2 receptor (CD25). Therefore it was hypothesized that Treg cells may efficiently consume IL-2 and suppress proliferation of responder T-cells by competing for IL-2 in the local environment. However, this proposal is not supported by the Treg cells present in IL$2 \mathrm{R}^{-/-}$mice that display similar in vitro suppressor activity to the wild-type counterpart (Fontenot et al., 2005a). Therefore IL-2 responsiveness is dispensable in Treg suppression, but is crucial for the maintenance of Treg cells.

\subsubsection{Role of CTLA-4 and GITR}

Cytotoxic T-lymphocyte antigen4 (CTLA-4) is generally expressed on activated $\mathrm{CD}^{+} \mathrm{T}$-cells, and it transduces a negative signal that results in dampening of T-cell activation following binding to CD80/CD86 ligands on APC (Oosterwegel et al., 1999). CD $25^{+}$Treg cells constitutively express CTLA-4, suggesting its importance in Treg biology. This hypothesis was supported in the study that the addition of anti-CTLA-4 antibody or its Fab fragment abrogated CD $25^{+}$Treg mediated suppression of responder $\mathrm{CD}^{+}{ }^{+}$T-cell proliferation in vitro (Takahashi et al., 2000). However, this result has been difficult to reproduce by other groups (Thornton and Shevach, 1998). The role of CTLA4 in Treg function is further supported by in vivo studies. For example, anti-CTLA-4 treatment abrogated Treg cells suppressing inflammatory bowel disease (IBD) (Read et $a l ., 2000)$, or induced AIG in BALB/c mice, suggesting that interaction between CTLA-4 and CD80/CD86 is required for Treg suppression effects. Although these findings indicated that the dispensable role of CTLA-4 signaling in CD25 ${ }^{+}$Treg suppressive 
function in vivo, the action site of anti-CTLA-4 antibody remains unclear, and it is possible that the antibody targeted the activated $\mathrm{CD} 4^{+} \mathrm{T}$-cells rather than the $\mathrm{CD} 25^{+}$Treg cells.

Like CTLA-4, GITR is also expressed on both Treg and activated CD4 $4^{+} \mathrm{CD} 25^{-} \mathrm{T}-$ cells (Shimizu et al., 2002). The ligand for GITR is expressed on endothelial cells, APC and dendritic cells. In vitro studies showed that the ability of Treg cells to suppress the proliferation of co-cultured CD25- T-cells was abrogated in the presence of anti-GITR antibody, indicating the important role of GITR stimulation in Treg cell suppressive function in vitro (McHugh et al., 2002; Shimizu et al., 2002). The action site of antiGITR antibody was determined using $\mathrm{CD} 25^{+}$Treg cells and responder T-cells from wildtype and GITR deficient mice. Ligation of GITR on CD25 responders, but not CD25 Treg cells, was required to abrogate suppression. Thus the interaction of GITR on effector T-cells with its ligands mediates resistance to suppression by Treg cells.

\subsubsection{Effects on APC}

Suppression may result from down-modulation of the capacity of APC to activate effector T-cells, or competition between CD25 ${ }^{+}$Treg cells and Tconv cells for costimulatory molecules expressed on APC. Several studies have reported that Treg cells could down-regulate the expression of co-stimulatory molecules CD80/CD86 by DCs (Cederbom et al., 2000; Houot et al., 2006; Kryczek et al., 2006; Serra et al., 2003). However, activation of $\mathrm{CD} 25^{+}$Treg suppressor function did not require co-stimulation by CTLA-4 interaction with CD80/86 (Thornton et al., 2004). CD25 ${ }^{+}$Treg cells were able to suppress $\mathrm{CD} 8^{+} \mathrm{T}$-cell proliferation in APC-free system in vitro, suggesting that APC are not required for suppression and the suppression may involve direct interaction between Treg and effector T-cells (Piccirillo and Shevach, 2001). Therefore competition for co-stimulation is unlikely to be a major mechanism of suppression. Recent studies have shown that lymphocyte-activation gene 3 (LAG-3) may block DC maturation. LAG-3, expressed on CD25 ${ }^{+}$Treg cells, is a CD4 homologue that binds MHCII molecules and has a negative regulatory function which is required for maximal Treg cell suppression (Huang et al., 2004; Workman and Vignali, 2005). Engagement of LAG3 to MHCII molecules expressed by immature DCs induces an immunoreceptor tyrosinebased activation motif (ITAM) mediated inhibitory signaling pathway, that suppresses DCs maturation (Liang et al., 2008). However, more work is required to investigate whether and how Treg mediate suppressive function through DCs.

\subsubsection{Thymic and Extrathymic Origin of Foxp $3^{+}$Treg}

\subsubsection{Thymic Development of Foxp $3^{+}$Treg Cells}

In 1969, it was observed that neonatal thymectomy at certain period of time (day three after birth) results in autoimmune damage of various organs (Nishizuka and 
Sakakura, 1969). Subsequent studies showed that d3Tx induced autoimmunity can be prevented by transfer of $\mathrm{CD}^{+}$T-cells or $\mathrm{CD} 4^{+} \mathrm{CD} 8{ }^{-}$thymocytes from syngeneic adult mice (Fowell and Mason, 1993; Sakaguchi et al., 1982), or more specifically, by transfer of CD25 $5^{+}$Treg cells isolated from normal adult mice (Asano et al., 1996). This suggests that $\mathrm{d} 3 \mathrm{Tx}$ mice intrinsically lack $\mathrm{CD} 25^{+}$Treg and provide a clue that Treg cells may originate in the thymus. It is found that nTreg develop in fetal thymic organ cultures (FTOC) (Carter et al., 2005; Kim and Rudensky, 2006). And GFP-Foxp3 knock-in mice revealed selection of T-cells into the Treg lineage at the transition from DP to SP thymocytes (Hsieh et al., 2006). Treg cells migration from the thymus to the periphery was documented by injection of FITC intrathymically (Papiernik et al., 1998). Furthermore, Itoh et al found that $\mathrm{CD} 25^{+} \mathrm{CD} 4^{+} \mathrm{CD} 8^{-}$thymocytes showed similar in vitro functional characteristics as their peripheral counterparts, such as anergic and suppressive responses to conventional T-cells (Itoh et al., 1999). In addition, $\mathrm{CD} 25^{+} \mathrm{CD} 4^{+} \mathrm{CD} 8^{-}$ thymocytes display a similar phenotype - expression of CTLA-4, GITR and OX40, as peripheral Treg cells (Itoh et al., 1999; Shimizu et al., 2002). Taking together, these data support the thymus is the site of nTreg generation. Mice deficient in all class II molecules did not develop $\mathrm{CD} 4^{+} \mathrm{CD} 25^{+} \mathrm{T}$-cells, while mice expressing MHC class II molecules only in the thymic cortex did (Bensinger et al., 2001). This indicates that the development of Treg cells requires interaction with self-antigen-MHCII complexes on the thymic APC.

It is not known whether Treg cells undergo positive and negative selection as conventional T-cells do. The induction of Foxp3 in nTreg requires CD28 signaling induced by CD 80 and CD86 expressed on antigen presenting cells, as Treg cells were markedly decreased in CD28 deficient and CD80/86 deficient mice (Salomon et al., 2000; Tai et al., 2005). Additionally, the lck-binding domain of CD28 cytoplasmic tail is critical for the induction of Foxp3, therefore suggesting that CD28 signaling is important in Treg differentiation in thymus (Tai et al., 2005). IL-2, IL-2R $\alpha$ (CD25) and IL-2R $\beta$ (CD122) are important in Treg development. Mice which lack any of them showed a significantly reduced number of thymic and peripheral CD25 $5^{+}$Treg cells and developed autoimmunity (Almeida et al., 2002; Malek et al., 2002; Papiernik et al., 1998). The autoimmune diseases could be controlled by reconstitution of these mice with normal CD25 ${ }^{+}$T-cells (Malek and Bayer, 2004). This suggests that IL-2 signaling was required for thymic development of CD25 $5^{+} \mathrm{T}$-cells. Whether TCR on Tregs has a higher affinity for self-antigen-MHC complexes than conventional T-cells is still controversial and is discussed below.

\subsubsection{Peripheral Generation of Foxp $3^{+}$Treg Cells}

One important question is whether thymus is the only place for the generation of Foxp $3^{+}$T-cells. A number of studies (both in vivo and in vitro) have revealed that Foxp $3^{+} \mathrm{T}$-cells can also be generated extrathymically from peripheral naive $\mathrm{CD} 4^{+}$Foxp $3^{-}$ T-cells, called adaptive Treg (iTreg). A variety of conditions favor iTreg conversion. For example, in vitro antigen-stimulation of naive T-cells in the presence of TGF- $\beta$ will induce iTreg (Apostolou and von Boehmer, 2004; Chen et al., 2003; Kretschmer et al., 
2005). In TGF- $\beta$ dependent in vitro Treg induction, IL-6, an acute phase protein induced during inflammation, dampens the conversion from naive T-cells and facilitates T-cell differentiation to the Th17 lineage in mice (Bettelli et al., 2006; Veldhoen et al., 2006). Genetic deletion or antibody blockage of IL-2 also promotes the differentiation of naive CD4 ${ }^{+}$T-cells into Th17 cells, suggesting that IL-2 facilitates the Treg differentiation but inhibits Th17 cell differentiation via signal transducer and activator of transcription 5 (STAT5) (Laurence et al., 2007). In addition, the vitamin A metabolite retinoic acid (RA), which is produced by a subset of DCs in the gut-associated lymphoid tissue, inhibits IL-6 mediated Th17 cell induction. In the presence of TGF- $\beta$, RA facilitates the differentiation of naive T-cells to Foxp ${ }^{+}$Treg cells (Benson et al., 2007; Coombes et al., 2007; Mucida et al., 2007; Sun et al., 2007). Furthermore, naive monoclonal HAspecific $\mathrm{CD}^{+}$T-cells were induced to express Foxp3 under the chronic low dose antigen (HA) stimulation. This suggests that suboptimal co-stimulation is critical for differentiation of iTreg (Apostolou and von Boehmer, 2004; Kretschmer et al., 2005). Gut-associated lymphoid tissue is a prominent in vivo site for iTreg generation because it is enriched in commensal bacteria and food antigens, and CD103 ${ }^{+} \mathrm{DCs}$, which produce RA and TGF- $\beta$, are potent inducers of iTreg. These dendritic cells are abundant in lamina propria (LP) and in the mesenteric lymph node (LN), helping to limit gut inflammation through the generation of iTreg.

Early evidence of peripheral conversion of naive $\mathrm{CD} 4^{+} \mathrm{T}$-cells into Foxp $3^{+} \mathrm{T}$ cells originated from adoptive transfer of polyclonal $\mathrm{CD} 4^{+} \mathrm{CD} 25^{-}$naive T-cells into lymphopenic mice. Part of the donor population became CD $25^{+}$Foxp $3^{+}$and acquired suppressive activity (Curotto de Lafaille et al., 2004). However, these CD $25^{+}$Foxp $3^{+}$ Treg cells might have originated from $\mathrm{CD} 25^{-} \mathrm{Foxp} 3^{+}$cells present in the initial population. To avoid this issue, mature TCR transgenic (Tg) Foxp3- T-cells on a $\mathrm{Rag}^{-/-}$background which lack a Foxp $3^{+}$component, were transferred into $\mathrm{Rag}^{-/-}$recipients that express agonist peptide under control of the Ig promoter. The injected cells segregated into CD25 $5^{+}(43.5 \%)$ and $\mathrm{CD} 25^{-}(56.5 \%)$. Both populations were anergic and suppressive (Apostolou et al., 2002). This suggests that mono-specific naïve T-cells can differentiate autonomously into antigen-specific Treg cells in peripheral lymphoid tissue.

However, the extent to which Foxp3- T-cells are converted to Foxp3 ${ }^{+}$Treg in both normal circumstances and autoimmunity remains unclear. In unimmunized mice, TCR repertoire analysis showed distinct populations among Treg and Tconv TCR (Hsieh et al., 2006; Lathrop et al., 2008; Wong et al., 2007b). Tracing the Foxp3- Tconv cells which were adoptively transferred into normal adult hosts confers different results. In one study, $5-12 \%$ of transferred CD $4^{+} \mathrm{CD} 25^{-} \mathrm{T}$-cells express CD25 (Liang et al., 2005), indicating a significant contribution of conversion to maintain the peripheral Treg population. However, another study showed that average of $0.5 \% \mathrm{CD}^{+}{ }^{+} \mathrm{Foxp} 3^{-} \mathrm{T}$-cells converted into Foxp $3^{+} \mathrm{T}$-cells, arguing that peripheral conversion plays a relatively small role in Treg pools (Lathrop et al., 2008). In autoimmune circumstances, sequencing of CDR3a motifs from Treg and Tconv cells in BDC2.5/NOD mice, which is a diabetes model, indicated that little or no conversion occurs in response to pancreatic auto-antigen (Wong et al., 2007a). Foxp $3^{-}$splenocytes adoptively transferred into congenic hosts followed by EAE induction did not convert into Foxp $3^{+}$T-cells in spleen and CNS, suggesting conversion 
was unlikely to occur following immunization of MOG/CFA (Korn et al., 2007). Collectively, the role of conversion to contribute to the peripheral Treg population is still ambiguous, and we will address this question using repertoire analyses (in Chapter 4) and studying specificity of a cohort of individual TCR (in Chapter 5).

\subsubsection{Specificity of Regulatory T-cells}

Affinity based model of Treg development propose that the increased strength of TCR and self-peptide-MHC complex favors the selection of Treg and these Treg cells have an increased affinity for self antigens. This is supported by studies with double transgenic mice (HA28 x TS1). When TCR transgenic mice bearing a TCR specific for epitope $\mathrm{S} 1$ derived from influenza hemagglutinin (HA) were crossed with mice expressing HA transgene, the S1-specific T-cells were not deleted and a large proportion expressed CD25 and functioned as Treg (Jordan et al., 2001). Furthermore, thymocytes bearing TCRs with low affinity to S1 did not develop into Treg, which suggested that the selection of Treg appeared to require a TCR with high affinity for self-antigen-MHC complex (D'Cruz and Klein, 2005; Jordan et al., 2001; Kawahata et al., 2002; Lohr et al., 2003; Walker et al., 2003). Hsieh et al. directly tested the specificity of Treg cells in vivo and in vitro. He retrovirally transfected different TCR $\alpha$ genes into conventional $\mathrm{CD}^{+} \mathrm{T}$ cells of TCli TCR $\beta$ transgenic $x$ Rag $^{-/-}$mice (TCli $\alpha \beta$ TCR is specific for foreign antigen human class II-associated Ii peptide), and thus recreated the TCRs derived from either CD25 or CD $25^{+}$population. The T-cells expressing Treg TCRs proliferated more rapidly than the transductants with Tconv TCRs upon adoptive transfer into lymphopenic hosts or in co-cultures with autologous APCs (Hsieh et al., 2004). Moreover, when the TCli TCR $\beta$ transgenic mice were crossed with Foxp3 deficient mice, the autoreactive Foxp $3^{-} \mathrm{CD} 25^{+} \mathrm{T}$-cells, but not Foxp $3^{-} \mathrm{CD} 25^{-}$cells, preferentially used TCRs found in the Treg cells in Foxp3 sufficient TCR $\beta$ transgenic mice (Hsieh et al., 2006). Taken together, these results implies that Treg cells express TCRs with increased affinity to self, and these TCRs use an overlapping repertoire with pathogenic autoimmune T-cells.

However, an alternative explanation of the Treg percentage increase in the HA28 $\mathrm{x}$ TS1 double transgenic system could be that Treg cells were more resistant to negative selection than their Foxp3- counterparts (Bonasio et al., 2006; Liston et al., 2003; van Santen et al., 2004). In experiments where the transgenic TCR was confronted with different levels of expression of tetracycline-regulatable neo-self antigen (MCC), the proportion of Treg cells increased without a change in absolute numbers. Thus, selective survival might be an alternative explanation for Treg differentiation. In addition, Treg cells can recognize foreign antigen derived from bacteria, viruses and parasites and neoantigens. And analyses of T-cell hybridomas derived from Treg and non-Treg TCRs found no evidence that Treg hybridomas preferably recognize self-antigens. Instead, Treg hybridomas recognized foreign MHC-peptide complexes as often as non-Treg cells (Pacholczyk et al., 2007). In conclusion, whether Treg cells possess higher affinity to self antigens than Tconv cells is still controversial, and requires further studies to determine the affinity range of Treg cells and self reactive Tconv cells. 
Another important issue is whether antigen specificity of Treg cells is required for suppressive function. In vitro studies indicate that $\mathrm{CD} 25^{+}$Treg cells, once activated, are capable of suppressing responder T-cells of disparate antigen specificity (Takahashi et al., 1998; Thornton and Shevach, 1998). However, in vivo studies link Treg specificity with their activity. Myelin basic protein (MBP) specific TCR transgenic Treg cells are more potent than non-transgenic Treg cells in EAE protection (Hori et al., 2002). Similar findings have been seen in nonobese diabetic (NOD) mice. In this study, in vitro expanded BDC2.5 TCR transgenic Treg cells that are specific for an islet antigen expressed in the granules of $\beta$ cells, suppressed diabetes in NOD Treg deficient mice better than expanded polyclonal NOD Treg cells (Tang et al., 2004). A similar result was seen in an autoimmune gastritis (AIG) model, in which antigen-specific induced Treg (iTreg) completely prevent AIG while polyclonal iTreg did not (Huter et al., 2008). In contrast, studies of a model of autoimmune uveitis failed to identify tissue specificity among involved Treg cells (Grajewski et al., 2006).

\subsubsection{Regulatory T-cells in MOG-EAE}

During autoimmunity, Treg cells substantially accumulate in affected organs. Early studies showed that reconstitution of nude mice with $\mathrm{CD} 4^{+} \mathrm{T}$-cells depleted for the $\mathrm{CD} 25^{+}$subpopulation resulted in the spontaneous development of autoimmune disease (Sakaguchi et al., 1995), while replenishment of the $\mathrm{CD}^{+} \mathrm{CD} 25^{+} \mathrm{T}$-cells prevented this. In EAE, CD25 ${ }^{+}$T-cells from naive C57BL/ 6 mice can inhibit both IFN- $\gamma$ production and proliferation of encephalitogenic cells from $\mathrm{MOG}_{35-55}$ immunized mice in vitro.

Adoptive transfer of these cells can ameliorate or abrogate the severity of EAE (Kohm et al., 2002; Mekala et al., 2005; Selvaraj and Geiger, 2008; Tang and Bluestone, 2006; Zhang et al., 2004). On the contrary, depleting functional Treg with anti-CD25 antibody (PC61) prior to immunization increases the severity of disease (McGeachy et al., 2005; Reddy et al., 2004; Stephens et al., 2005).

During the course of EAE, Treg cells have been identified in the inflamed CNS by flow cytometry and histological analysis (Kohm et al., 2006; Korn et al., 2007; Liu et al., 2006; McGeachy et al., 2005). Importantly, the accumulation of Treg cells peaks at the recovery phase. About $30 \%$ of these cells can produce IL-10 and these cells displayed potent suppressive capacity ex vivo (McGeachy et al., 2005). The origination of these CNS-infiltrating Treg cells is still a puzzle. One study demonstrated that transferred pathogenic T-cells could convert to Foxp $3^{+}$phenotype once localized in the CNS (Liu et al., 2006). However, tracking of transferred MOG-specific Tconv cells in a passive model, and tracking of transferred polyclonal CD4 ${ }^{+} \mathrm{Foxp}^{-} \mathrm{T}$-cells in an active model failed to identify the conversion (Davidson et al., 2007; Korn et al., 2007). The previous conversion phenomenon might come from expansion of an already existing Foxp $3^{+}$population within the transferred "pathogenic" T-cells. Thus, whether Tconv cells can convert to Treg during EAE is still controversial. Another feature of these CNS-infiltrating Foxp ${ }^{+}$Treg cells is their remarkable proliferative capacity, and this striking proliferative state is observed in CNS but not in draining lymphoid tissues 
(O'Connor et al., 2007). Treg cells are usually anergic when cultured in vitro, and it seems the inflammatory environment really promotes their expansion.

Another important question is what antigens CNS-infiltrating Treg cells recognize. Isolated Treg cells quickly revert to the classic anergic state in vitro, making antigen identification technically challenging to address. Although some studies showed Treg cells may recognize foreign antigens (Pacholczyk et al., 2007), substantial evidence indicates that the repertoire also bears an overrepresentation of self-specific TCRs (Andersson et al., 2007; Hsieh et al., 2004; Hsieh et al., 2006; Jordan et al., 2001; Romagnoli et al., 2002). Similarly, MOG-specific Treg cells were identified by tetramer staining in Foxp3-GFP reporter mice (Korn et al., 2007; Reddy et al., 2004; Reddy et al., 2005). Likewise Foxp $3^{+}$cells derived from the CNS of MOG immunized mice with EAE expanded greatly alter the addition of MOG-peptide to the culture (O'Connor et al., 2007). Those studies suggest that CNS-infiltrating Treg cells in MOG-EAE contain at least a portion of MOG-reactive cells.

Cross-reactivity is also common in T-cell receptors. For example, EAE spontaneously developed in MOG deficient mice expressing a MOG-specific TCR (2D2). This paradox was explained by the finding that 2D2 cells are cross reactive with a neuronal cytoskeletal self antigen, neurofilament-M (NF-M) (Krishnamoorthy et al., 2009). Even though 2D2 cell belongs to Tconv family, it is possible that CNS-infiltrating Treg cells in MOG-EAE also have cross-specificity for additional antigens.

\subsubsection{Repertoire Analysis of Treg Cells}

Although Treg cells are only a small fraction of CD4 ${ }^{+}$T-cells (5-10\%), they can respond to a broad spectrum of antigens, both self and foreign. How does such a limited population recognize so many different specificities? Analyses of $\alpha \beta$ TCR diversity on $\mathrm{CD} 4{ }^{+} \mathrm{CD} 25^{+} \mathrm{T}$-cells showed that their repertoire is diverse. Early studies showed $\alpha \beta T C R$ repertoire of $\mathrm{CD} 4{ }^{+} \mathrm{CD} 25^{+} \mathrm{T}$-cells is more diverse than the repertoires of natural killer T-cells (NK T-cells) (Olivares-Villagomez et al., 2000; Takahashi et al., 1998). The comparison of the use of TCR V $\beta$ or V $\alpha$ on Tconv and Treg cells revealed similar contributions of variable subfamilies to the two subpopulations of T-cells in both mice and humans (Fujishima et al., 2005; Kasow et al., 2004; Pacholczyk et al., 2002; Romagnoli et al., 2002; Takahashi et al., 1998). Further analysis of CDR3 size distribution showed that the diversity of Treg CDR3 $\beta$ was as broad as Tconv subsets (Fujishima et al., 2005; Kasow et al., 2004).

Since each individual can generate millions of different TCRs, CDR3 length distribution analysis is not sensitive enough to determine the identity of individual TCRs. A more detailed analysis is to sequence individual TCR CDR $3 \alpha$ or $\beta$ regions amplified by reverse transcription-polymerase chain reaction (RT-PCR) (Apostolou et al., 2002; Hsieh et al., 2004; Hsieh et al., 2006; Pacholczyk et al., 2006; Wong et al., 2007b). Most CDR3 sequence analyses were performed on TCR $\beta$ transgenic mice, in which all T-cells express a single rearranged TCR $\beta$ chain paired with variable TCR $\alpha$ chains. Therefore, 
the CDR3 $\alpha$ can be used as an identifier of individual T-cell clones. The direct comparison of CDR3 $\alpha$ sequences among thymic or peripheral Treg and Tconv cells suggest that both populations have very diverse repertories. Interestingly, reduction in the diversity of peptide-MHC complexes presented in thymus did not restrict the Treg repertoire diversity (Apostolou et al., 2002; Pacholczyk et al., 2006; Wong et al., 2007b). This correlated with previous work showing that the proportion of $\mathrm{CD} 4{ }^{+} \mathrm{CD} 25^{+}$cells among $\mathrm{CD}^{+} \mathrm{SP}$ thymocytes remains constant in mice expressing different diversities of peptides bound with MHCII molecules (Pacholczyk et al., 2002). This might suggest that the selection of Treg cells is not exclusively dependent on specific high-affinity to particular peptide-MHC complexes but rather Treg are selected more loosely as natural $\mathrm{CD} 4{ }^{+} \mathrm{T}$-cells. Or alternatively, the diversity of Treg repertoire is reduced in these mice with limited thymic antigen diversity, but is still too diverse to be detected by current techniques. Because fewer DP thymocytes will pass through positive selection as a result of the reduced diversity of peptide-MHC complexes, not only the Treg repertoire but also Tconv repertoire will shrink, probably to the same extent, which leads to a similar proportion of Treg cells developing.

Analyses of Treg and Tconv repertoires also demonstrates that the Treg and Tconv repertoires have partial sequence overlap, ranging from 10 to $42 \%$, depending on different experimental strategies (Hsieh et al., 2004; Hsieh et al., 2006; Lathrop et al., 2008; Pacholczyk et al., 2006; Pacholczyk et al., 2007). In mice expressing unmanipulated polyclonal self antigens on MHCII molecules $\left(\mathrm{A}^{\mathrm{b}} \mathrm{wt}\right)$, the overlap is approximately 10-20\% (Apostolou et al., 2002; Hsieh et al., 2004; Hsieh et al., 2006; Pacholczyk et al., 2006). Whereas in "single-peptide" mice whose MHCII molecules are loaded with exclusively one peptide, the overlap increased to $38 \%$ ( $\mathrm{A}^{\mathrm{b}} \mathrm{Ep}$ mice) and $42 \%$ (H2-DM-deficient mice) (Apostolou et al., 2002; Pacholczyk et al., 2006; Wong et al., 2007b). The correlation of increasing overlap with decreasing diversity of selecting ligands implies selection of Treg cells is not directly linked to the high selfreactivity,(Pacholczyk and Kern, 2008) because if Treg cells possess higher selfreactivity, one should expect more limited overlap in "single-peptide" mice.

This type of repertoire analysis can also be used to survey the interconversion between Treg and Tconv cells during the course of autoimmunity. For example, in a diabetes model, only limited Treg and Tconv cell TCR sequence overlap was seen in islets, suggesting that the two cell types were not interconverting at the site of inflammation (Wong et al., 2007a).

Comparison of the TCR repertoire of Foxp $3^{+}$Treg cells present in the thymus and the ones present in the periphery showed that the peripheral Treg cells resembled those of thymic precursors, and differed from Foxp3- T-cells (Apostolou et al., 2002; Hsieh et al., 2006; Pacholczyk et al., 2006; Wong et al., 2007b). These findings support the view that the peripheral Treg cells are derived from thymic precursors. On the other hand, the peripheral Treg repertoire does reshape itself somewhat. For example, TCRs frequently found in the thymus are also represented in the periphery, but their dominance may be altered (Apostolou et al., 2002; Correia-Neves et al., 2001; Hsieh et al., 2006; Pacholczyk et al., 2006; Wong et al., 2007b). In addition, peripheral interconversion 
between Treg and Tconv cells is observed, suggesting this conversion process could contribute to changes in the peripheral Treg repertoire, compared with thymic Treg repertoire. The peripheral conversion may also play a role in maintenance of the peripheral Treg cell population, and might be an important mechanism limiting inflammation induced immunopathology, especially in gut - associated lymphoid tissues (Coombes et al., 2007; Lathrop et al., 2008; Liang et al., 2005). However, to what extent that the interconversion contributes to peripheral Treg cells in autoimmunity is still uncertain.

\subsection{Summary}

Regulatory T-cells, which are recognized as $\mathrm{CD} 4^{+} \mathrm{CD} 25^{+} \mathrm{Foxp} 3^{+} \mathrm{T}$-cells, are critical for immune homeostasis. They are naturally present in the normal immune system as a functionally distinct T-cell subpopulation, and their deficiency produces autoimmune disease (Sakaguchi et al., 1995). Adoptively transferred Treg cells can abrogate or prevent organ specific autoimmunity (Kohm et al., 2002; Mekala et al., 2005; Selvaraj and Geiger, 2008; Tang and Bluestone, 2006; Zhang et al., 2004). Thymus is the major site of producing Treg cells. Besides that, peripheral interconversion between Treg and Tconv cells is also observed (Coombes et al., 2007; Lathrop et al., 2008; Liang et al., 2005), though in different degrees. During EAE, Treg cells are often found in substantial number within affected organs (CNS), and the accumulation is correlated with the recovery phase (Korn et al., 2007; Reddy et al., 2004; Yu et al., 2005). However, the origination of these Treg cells and responsiveness difference to Tconv cells are still unclear. In order to solve this issue, we developed a retrogenic mouse model of EAE, combined both repertoire analysis and studies of a large cohort of individual TCR. We found that Treg and Tconv cell TCR repertoires have limited overlap within the periphery and CNS in mice with EAE, (Chapter 4) and MOG-specific Treg and Teff cells possess distinct CDR3 $\beta$ characters but similar sensitivity and fine specificity for MOG autoantigen (Chapter 5). 


\section{CHAPTER 2. MATERIALS AND METHODS}

\subsection{Methods of TCR Repertoire Analysis}

\subsubsection{Vector Construction for TCR $\alpha$}

Generation of MSCV-IRES-GFP vector was described previously (Persons et al., 1997). The GFP was replaced by CFP. To generate different TCR $\alpha$ constructs, the $\alpha$ chain from 1MOG244.2, 2MOG10, 1MOG9, 1MOG23, and 1MOG213 MOG-specific TCR (Alli et al., 2008) were PCR amplified and inserted into EcoRI/XhoI cloning sites in the vector (Figure 2-1).

\subsubsection{Generation of 1MOG244.2TCR $\alpha$ Retrogenic Mice}

\subsubsection{Retroviral Production and Cellular Transduction}

Ten $\mu \mathrm{g}$ of the 1MOG244.2TCR $\alpha$ construct DNA and 10 $\mu \mathrm{g}$ of retrovirus helper DNA constructs PEQPAM were co-transfected into 293T-cells by calcium phosphate precipitation as has been described (Geiger et al., 2001). Briefly, 24 hours prior to transfection, $3 \times 10^{6}$ 293T-cells were plated on a $10 \mathrm{ml}$ tissue-culture treated Petri dish. On the day of transfection, four hours prior to transfection, cells were approximately $70 \%$ confluent. DNA mix was prepared using $10 \mu \mathrm{g}$ construct DNA and $10 \mu \mathrm{g}$ PEQPAM plasmid, $50 \mu 12.5 \mathrm{M} \mathrm{CaCl}_{2}$ and $450 \mu \mathrm{l}$ water at room temperature (RT). This mix was then added to $500 \mu \mathrm{l}$ HPBS ( $\mathrm{pH}$ 7.2) dropwise while vortexing and incubated 1 minute at RT. The whole mixture was added onto $293 \mathrm{~T}$-cells and incubated at $37^{\circ} \mathrm{C}$. At 16 hours, the cells were washed with PBS to remove $\mathrm{Ca}^{2+}$ and cultured in Dulbecco modified Eagle medium (DMEM) with 10\% fetal calf serum (FCS), $100 \mathrm{U} / \mathrm{ml}$ penicillin $\mathrm{G}, 100 \mu \mathrm{g} / \mathrm{ml}$ streptomycin, $292 \mu \mathrm{g} / \mathrm{ml} \mathrm{L-glutamine} \mathrm{(Invitrogen} \mathrm{Life} \mathrm{Technologies)} \mathrm{at} 37^{\circ} \mathrm{C}$. At the same time $3 \times 10^{5} \mathrm{GP}+\mathrm{E} 86$ cells were plated in a 10ml Petri dish. 24 hours later, supernatant from 293T-cells was collected, centrifuged at 3000 rotrations per minute (rpm) for 7 minutes and the supernatant was used to infect GP+E86 retroviral producer cells in the presence of $6 \mu \mathrm{g} / \mathrm{ml}$ polybrene (hexadimethrine bromide; Sigma-Aldrich). $10 \mathrm{ml}$ fresh medium was added to $297 \mathrm{~T}$-cells, and ready for next time infection. GP+E86 cells were infected twice daily for five days. The GP+E86 cell line was expanded in culture for establishing stable retroviral vector producer lines.

\subsubsection{Retroviral Transduction and Transplant of Bone Marrow Cells}

GFP-Foxp3 gene-targeted mice were provided by A. Rudensky (U. Washington). $\mathrm{Tcr}^{-/-}$mice were obtained from The Jackson Laboratory (Bar Harbor, ME). These two strains were bred to generate GFP-Foxp3 $\mathrm{x} \mathrm{Tcr}^{-/-}$mice. DNA genotyping and flow 


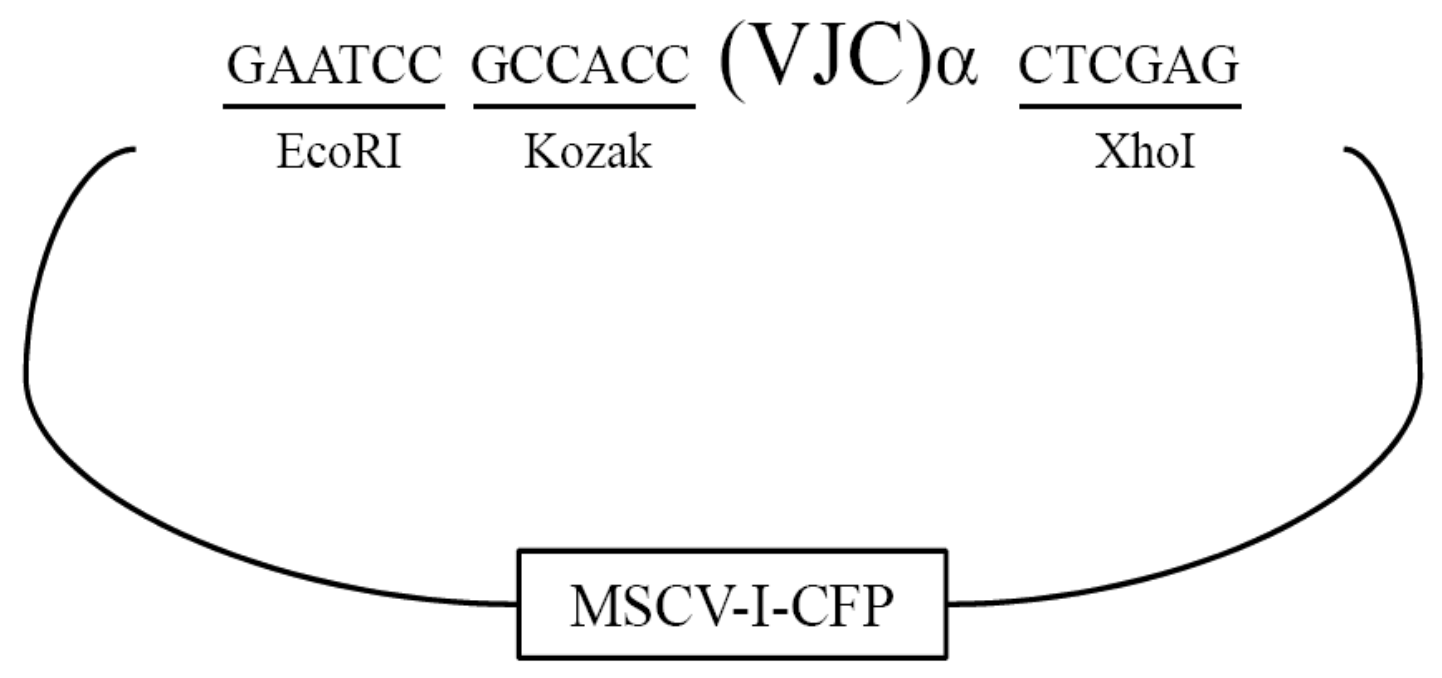

Figure 2-1. Structure of retroviral constructs. TCR $\alpha$ cDNA sequences were PCR amplified with primers containing EcoRI and XhoI sites, and subcloned into the MSCVI-CFP retroviral vectors. 
cytometry were performed for screening mice with GFP-Foxp3 gene and TCR $\alpha^{-/-}$gene. Genome DNA from each mouse was extracted by Gentra Puregene Mouse Tail Kit (Qiagen). Foxp3 gene was PCR amplified with CW78a and CW78b primers (CW78a, 5' - TGCCTCTGACAAGAACCCAATGC; CW78b, 5'-

GGCAGGGGGTTCAAGGAAGAAG). Nuclease-free water, dNTP mix, buffer with $\mathrm{MgCl} 2$, Q buffer (Qiagen), Taq polymerase and tail DNA were added to make total of $50 \mu 1$ PCR mix. Reactions were carried out using the following PCR parameters: $95^{\circ} \mathrm{C}$ for initial $3 \mathrm{~min}, 30 \mathrm{cycles}$ of $95^{\circ} \mathrm{C}$ for $30 \mathrm{sec}, 55^{\circ} \mathrm{C}$ for $30 \mathrm{sec}$, and $72^{\circ} \mathrm{C}$ for $2.5 \mathrm{~min}$, followed by $72^{\circ} \mathrm{C}$ for $6 \mathrm{~min}$. The products were analyzed by $1 \%$ agarose gel electrophoresis. The knock-in band is $927 \mathrm{bp}$ while the WT is $210 \mathrm{bp}$. To screen TCR $\alpha^{-/-}$ mice, peripheral blood was collected and RBCs were lysed. Isolated cells were stained with anti-TCR $\beta$ and anti-B220 antibody, and analyzed by flow cytometry.

The production of retrogenic mice has been previously described (Holst et al., 2006a; Holst et al., 2006b). Briefly, bone marrow cells were harvested from the femurs of GFP-Foxp3 $\mathrm{x}$ Tcr ${ }^{-/-}$mice $48 \mathrm{hr}$ after the administration of $0.15 \mathrm{mg} 5$-fluorouracil $\mathrm{g}^{-1}$ body weight, cultured in complete Click's medium (Invitrogen) with 20\% FCS, IL-3 (20 $\left.\mathrm{ng} \mathrm{ml}^{-1}\right)$, IL-6 $\left(50 \mathrm{ng} \mathrm{ml}^{-1}\right)$, and stem cell factor $\left(50 \mathrm{ng} \mathrm{ml}^{-1}\right)$ for $48 \mathrm{hr}$ and then cocultured for an additional $48 \mathrm{hr}$ with irradiated (1200 rad) GP+E86 retrovirus producer cells. Transduction efficiency was confirmed by flow cytometry with CFP. Transduced progenitor cells were harvested, resuspended in PBS with $2 \%$ FCS and heparin (10unit/mouse) in total volume of $500 \mathrm{ul} / \mathrm{mouse}$, and injected into sublethally irradiated $(450 \mathrm{rad}) \mathrm{Tcr}^{-/-}$recipients at a ratio of 1.5 recipient mice per bone marrow donor.

To test the engraftment of these retrogenic mice, peripheral blood was collected 6-8 weeks after transplantation. Red blood cells were lysed with Gey's solution (Sigma). Isolated cells were stained with anti-CD4 APC and anti-TCR $\beta$ PE antibody, and analyzed by flow cytometry.

\subsubsection{EAE Induction and Clinical Evaluation}

MOG $_{35-55}$ peptide (MEVGWYRSPFSRVVHLYRNGK) was synthesized by the St.Jude Hartwell Center for Biotechnology and HPLC purified prior to use. $2 \mathrm{mg} / \mathrm{ml}$ $\mathrm{MOG}_{35-55}$ was resuspended in water, and mixed with an equal volume of complete Freund's adjuvant (CFA) containing 4mg/ml H37RA. Emulsion was achieved by vortexing the mixture for 1 hour at room temperature.

EAE was induced on retrogenic mice by s.c. injection of $100 \mu \mathrm{g}$ of $\mathrm{MOG}_{35-55}$ peptide emulsified in CFA containing $4 \mathrm{mg} / \mathrm{ml}$ Mycobacterium tuberculosis H37RA (Difco). Two hundred nanograms of pertussis toxin (List Biological Laboratories) was administered i.v. on days 0 and 2 . Clinical scoring was as follows: 1 , limp tail; 2 , hind limb paresis or partial paralysis; 3, total hind limb paralysis; 4 , hind limb paralysis and body/front limb paresis/paralysis; 5 , moribund. 


\subsubsection{Cell Isolation}

\subsubsection{Preparation of the Splenocyte Suspension}

1MOG244.2 retrogenic mice were sacrificed, spleens were removed and placed individually in $6 \mathrm{~cm}$ diameter dishes. $5 \mathrm{ml}$ PBS with $2 \%$ FCS were added and completely covered the bottom of the dish. Cells from the splenic capsule were squeezed through the $70 \mu \mathrm{m}$ nylon mesh by gently mashing spleens with the rubber end of a plunger from a $3 \mathrm{cc}$ syringe. A single cell suspension was created and collected into a $50 \mathrm{ml}$ conical tube (on ice). $5 \mathrm{ml}$ PBS with $2 \%$ FCS was added to the dish and the whole process was repeated twice until the splenic capsules were white, indicating complete release of the cells. The splenocytes were washed by adding $20 \mathrm{ml}$ buffer and centrifuged at $350 \mathrm{~g}$ for $7 \mathrm{~min}$ at $4^{\circ} \mathrm{C}$ (Eppendorf centrifuge 5804R with a type A-4-44 swinging bucket rotor).

The supernatant was aspirated and gently resuspended in $3 \mathrm{ml} /$ spleen Gey's solution in room temperature for two minutes. The cells were then washed with $10 \mathrm{ml}$ PBS buffer and centrifuged again. Splenocytes were resuspended and passed through a $70 \mu \mathrm{m}$ nylon mesh strainer into a new $50 \mathrm{ml}$ conical tube. The cell suspension from individual mice was pooled in same experiments. Spleens were prepared individually for TCR V $\beta$ usage analysis, and pooled for TCR CDR3 $\beta$ repertoire survey.

\subsubsection{Preparation of the Thymocytes and Lymphocytes Cell Suspension}

Mice were sacrificed by $\mathrm{CO}_{2}$ asphyxiation and their thymi, inguinal lymph nodes (LNs), axillary LNs, bronchial LNs, superficial and deep cervical LNs, and mesenteric LNs were collected. Similar to splenocytes, thymocytes and lymphocytes were collected by passing through $70 \mu \mathrm{m}$ cell meshes, and washed twice with PBS. Cell suspensions were centrifuged at $350 \mathrm{~g}$ for 10 minutes, pellets resuspended in staining buffer, and filtered with a $70 \mu \mathrm{m}$ cell strainer.

\subsubsection{Isolation of CNS-infiltrating T-lymphocytes}

Five to six $1 \mathrm{MOG} 244.2$ retrogenic mice were immunized with $\mathrm{MOG}_{35-55}$ peptide. On day 23-25, the peak phase of EAE disease, the mice were anesthetized with a lethal dose of Avertin $(0.5 \mathrm{ml}$ of $2.5 \%)$ delivered by intraperitoneal injection. When the mice were unresponsive to stimulus (no reflex with paw pressure), they were placed on a polystyrene board and pinned down. The abdomen was opened and spleen was gently removed. Then the heart was exposed by cutting up both sides of the rib cage and the diaphragm. The right atrium was cut open with sharp small scissors, a $27^{1 / 2}$ g needle was immediately inserted into the left ventricle and $10 \mathrm{ml}$ chilled PBS slowly pushed into the left ventricle. White lungs indicated a successful perfusion. The detached head was opened by peeling each side of skull back away, and brain was gently removed. The 
vertebra was dissected and the lumen was opened, and the spinal cord was collected with tweezers.

The CNS tissue was minced and transferred to a 6-well petri dish, digested with $3 \mathrm{ml}$ of $2.5 \mathrm{mg} / \mathrm{ml}$ collagenase (Worthington Biochemical) and $1 \mathrm{mg} / \mathrm{ml}$ deoxyribonuclease (Sigma) in click's medium for 25 minutes at $37^{\circ} \mathrm{C}$ followed by mechanical

disaggregation. The disrupted CNS was transferred to a $70 \mu \mathrm{m}$ nylon cell strainer and forced through the screen with a $3 \mathrm{cc}$ plunge. The suspension was centrifuged at $400 \mathrm{~g}$ for 10 minutes at room temperature. The pellet was resuspended in $4 \mathrm{ml}$ of $80 \%$ Percoll (Amersham Pharmacia biotech) and underlayed in $4 \mathrm{ml}$ of $40 \%$ Percoll. $2 \mathrm{ml}$ of $100 \%$ Percoll was used to underlay this. This gradient was carefully centrifuged at $1200 \mathrm{~g}$ for 20 minutes at $20^{\circ} \mathrm{C}$. The cells between the $40 \%$ and $80 \%$ interface were collected, washed twice and resuspended in staining buffer.

\subsubsection{Cell Counting}

A $10 \mu 1$ cell suspension was mixed with $90 \mu 1$ of PBS, and $100 \mu 1$ trypan blue solution was added and mixed thoroughly. A $10 \mu 1$ sample was transferred to a hemocytometer chamber. All viable cells were counted (non-viable cells stain blue, viable cells remain opaque) in the four corner squares. The cell concentration per $\mathrm{ml}$ was determined using the following calculation.

Cells per $\mathrm{ml}=$ the average count per square $\mathrm{x}$ the dilution factor $\mathrm{x} 10^{4}$

\subsubsection{Flow Cytometry}

\subsubsection{Flow Cytometry (FACS) Analysis of 1MOG244.2 Retrogenic Mice}

Eight weeks after hematopoietic progenitor cell (HPC) transfer, five to ten retrogenic mice were sacrificed. Thymocytes were isolated and a single cell suspension prepared. $10 \times 10^{6}$ cells were counted and resuspended in $1 \mathrm{ml}$ cell staining buffer $(500 \mathrm{ml}$ PBS and $10 \mathrm{ml} \mathrm{FCS} \mathrm{and} 5 \mathrm{ml} \mathrm{10 \%} \mathrm{sodium} \mathrm{azide).} 5 \mu \mathrm{g} / \mathrm{ml}$ purified anti-mouse CD16/CD32 antibody specific for Fc $\gamma$ R III/I (Miltenyi Biotec) was added and incubated for 10 minutes to block nonspecific immunofluorescent staining. The cells were stained with a combination of antibodies, which contained anti-CD4 (clone L3T4), anti-CD8 (clone 53-6.7), anti-TCR $\beta$ (clone H57-597), anti-CD44 (clone IM7), anti-CD25 (clone PC61). After 20 minutes incubation on ice, the cells were washed three times with $3 \mathrm{ml}$ of cell staining buffer. The cell pellet was resuspended in $0.5 \mathrm{ml}$ of cell staining buffer and analyzed by flow cytometry (BD FACSCalibur).

A similar preparation was performed with retrogenic splenocytes and CNSinfiltrating T-cells, but stained with two sets of antibodies. One set contained anti-CD2 (clone RM2-5), anti-TCR $\beta$ (clone H57-597), anti-CD25 (clone PC61), and anti-CD4 
(clone L3T4). The other set contained anti-CD69 (clone H1.2F3), anti-TCR $\beta$ (clone H57-597), anti-CD103 (clone M290), anti-CD5 (clone 53-7.3), and anti-CD4 (clone L3T4).

\subsubsection{V 3 Staining of 1MOG244.2 Retrogenic Mice}

Splenocytes and CNS-infiltrating T-cells were distributed into 14 wells (96-well round bottom plate). Monoclonal antibody specific for CD4 (clone L3T4) and CD8 (clone 53-6.7) were added equally. TCR $\beta$ (clone H57-597), TCR V $\beta 2$ (clone B20.6),

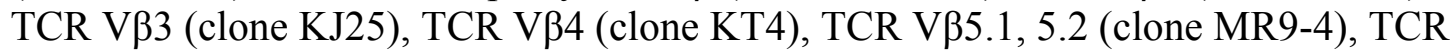
V $\beta 6$ (clone RR4-7), TCR V $\beta 7$ (clone TR310), TCR V $\beta 8.1,8.2$ (clone MR5-2), TCR V $\beta 8.3$ (clone 1B3.3), TCR V $\beta 10^{\mathrm{b}}$ (clone B21.5), TCR V $\beta 11$ (clone RR3-15), TCR V $\beta 12$ (clone MR11-1), TCR V $\beta 14$ (clone 14-2) were added sequentially. After incubation and wash, cells were analyzed by flow cytometry (BD FACSCalibur).

\subsubsection{Cell Separation by Flow Cytometry}

At the indicated time points after EAE induction, 5-6 mice/cohort were sacrificed, spleens were removed, the circulation perfused with PBS, and brain and spinal cord removed. T-cells were isolated from CNS tissue by density centrifugation as described above. Isolated cells were stained with CD4-specific $\mathrm{Ab}$ and immediately sorted into Tconv $\left(\mathrm{CD}^{+} \mathrm{GFP}^{-}\right)$and Treg $\left(\mathrm{CD}^{+} \mathrm{GFP}^{+}\right)$populations with a MoFlo (DakoCytomation) or iCyt reflection (iCyt, Champaign, ILL) cell sorter.

\subsubsection{RNA Isolation and $c D N A$ Transcription}

Total RNA was extracted from sorted splenocytes and CNS-infiltrating lymphocytes using RNeasy Protect MiniKit (QIAGEN), according to the manufacturer's instructions. The RNA concentration was determined by NanoDrop ND-1000 spectrophotometer.

cDNA was immediately generated with the Omniscript RT kit (QIAGEN). Oligo dT, RNase inhibitor, Omniscript reverse transcriptase, 10x buffer, dNTP mix, RNase-free water were mixed with RNA, and incubate at $37^{\circ} \mathrm{C}$ for 1 hour. The cDNA products were kept at $-20^{\circ} \mathrm{C}$ for long term storage.

\subsubsection{Polymerase Chain Reaction (PCR) Amplification}

cDNA was PCR amplified with either $\mathrm{C} \beta$ - or J $\beta 2.7$ - specific primers $\left(\mathrm{C} \beta, 5^{\prime}\right.$ CAAGGAGACCTTGGGTGGAGTCACATT; J $\beta 2.7,5$ '-

TAAAACCGTGAGCCTGGTGCCGG) and a V $\beta 8.2$ - primer (V $\beta 8.2,5$ 'ATGTCTAACACTGCCTTCCCTGACCC). A bulk reagent tube was created and 
distributed, which contained nuclease-free water, dNTP mix, buffer with $\mathrm{MgCl}_{2}$ and primers. Then cDNA and high fidelity polymerase (Roche) were added and tubes kept on ice. Five identical and independent PCR reactions were performed with each sample per primer pair. And a reaction without any cDNA was prepared as a negative control. The PCR program (Eppendorf Mastercycler gradient) was started, and tubes were placed into the machine once the temperature reached $95^{\circ} \mathrm{C}$. Touchdown PCR was used to avoid amplifying nonspecific sequences. The programs were as follows (Table 2-1).

To prevent PCR contamination between experiments, cDNA preparation and PCR post-amplification processing were performed in separate areas and using separate equipment. A laminar flow cabinet equipped with UV lamp was also used. Water, dNTP, buffer, tubes, pipette and tips etc were UV irradiated for 15 minutes immediately before use. Fresh gloves, pipette tips with aerosol filters and different sets of pipette were also required. A negative control was always included, in which the reaction mixture did not have DNA template.

\subsubsection{Agarose Gel Electrophoresis and PCR Product Purification}

A $1 \%$ agarose gel was prepared, and ethidium bromide added after the gel solution cooled to $60^{\circ} \mathrm{C}$. A $5 \mu 1$ aliquot from each PCR product was mixed with loading buffer and loaded into the gel wells, running at $120 \mathrm{~V}$ for 30 minutes. The products were examined briefly with a UV transilluminator (Figure 2-2). A positive reaction contained a $\sim 400$ bp fragment. Remaining products underwent a second round of gel electrophoresis. The $\sim 400 \mathrm{bp}$ DNA fragments were excised and purified with the QIAquick Gel Extraction Kit, according to the protocol.

Table 2-1. PCR program used to amplify CDR3 region.

\begin{tabular}{|c|c|}
\hline VB8.2- J $\beta 2.7$ & $\mathrm{~V} \beta 8.2-\mathrm{C} \beta$ \\
\hline 1. $95^{\circ} \mathrm{C} 2: 00 \mathrm{~min}$ & 1. $95^{\circ} \mathrm{C} 2: 00 \mathrm{~min}$ \\
\hline 2. $95^{\circ} \mathrm{C} 0: 30 \mathrm{~min}$ & 3. $95^{\circ} \mathrm{C} 0: 30 \mathrm{~min}$ \\
\hline 3. $75^{\circ} \mathrm{C} 0: 30 \mathrm{~min}$ & 4. $65^{\circ} \mathrm{C} 0: 30 \mathrm{~min}$ \\
\hline 4. $-1^{\circ} \mathrm{C} 0: 00 \mathrm{~min}$ & 5. $-1{ }^{\circ} \mathrm{C} 0: 00 \mathrm{~min}$ \\
\hline 5. $72^{\circ} \mathrm{C} 0: 30 \mathrm{~min}$ & 6. $72^{\circ} \mathrm{C} 0: 30 \mathrm{~min}$ \\
\hline 6. Goto 2 repeat 10 cycles & 7. Goto 2 repeat 10 cycles \\
\hline 7. $95^{\circ} \mathrm{C} 0: 30 \mathrm{~min}$ & 8. $95^{\circ} \mathrm{C} 0: 30 \mathrm{~min}$ \\
\hline 8. $65^{\circ} \mathrm{C} 0: 30 \mathrm{~min}$ & 9. $55^{\circ} \mathrm{C} 0: 30 \mathrm{~min}$ \\
\hline 9. $72^{\circ} \mathrm{C} 0: 30 \mathrm{~min}$ & $10.72^{\circ} \mathrm{C} 0: 30 \mathrm{~min}$ \\
\hline 10. Goto 6 repeat 20 cycles & 11. Goto 6 repeat 20 cycles \\
\hline 11. $72{ }^{\circ} \mathrm{C} 10: 00 \mathrm{~min}$ & $12.72^{\circ} \mathrm{C} 10: 00 \mathrm{~min}$ \\
\hline
\end{tabular}




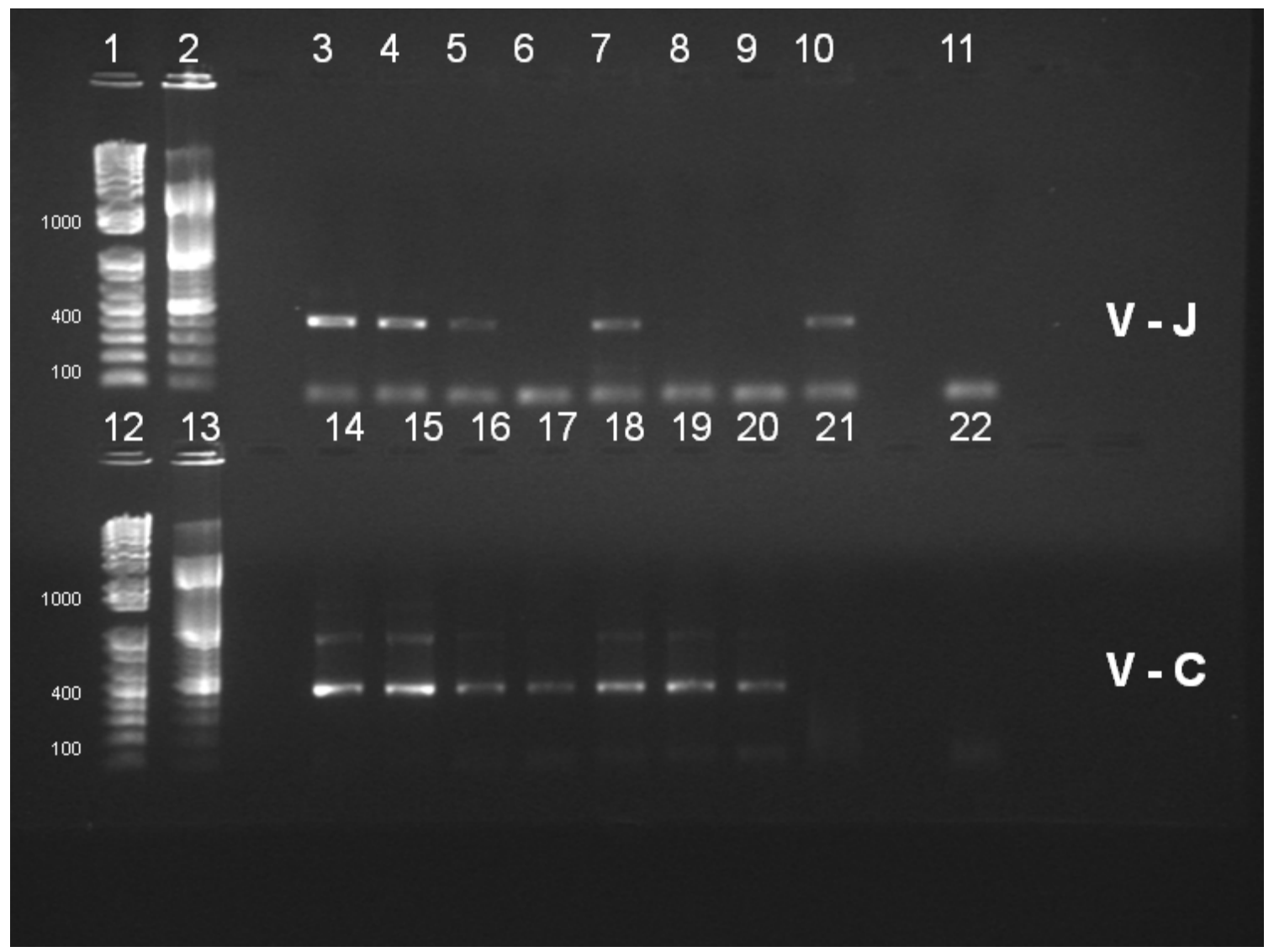

Figure 2-2. PCR amplification of CDR3 region. Lane 1 and 12: $1 \mathrm{~kb}$ plus ladder, lane 3 and 10: 100bp ladder, lane 14-21: amplified with V $\beta 8.2-J \beta 2.7$ primer, lane 11 and 22: negative control. 


\subsubsection{TA Cloning}

A deoxyadenosine (A) was introduced to the 3' ends of the purified DNA fragments. Briefly, gel purified PCR products were mixed with dATP, $25 \mathrm{mM} \mathrm{MgCl}_{2}$ and Taq polymerase (Promega), and incubate at $72^{\circ} \mathrm{C}$ for $10 \mathrm{~min}$. The products are then mixed with salt solution and pCR2.1 - TOPO vector (Invitrogen) and incubated at RT for 5 minutes. $2 \mu 1$ of the TOPO cloning reaction was added into a vial of One Shot Chemically Competent E. coli and mixed gently. After a 30 minute incubation on ice, the cells were heat shocked for 30 seconds at $42^{\circ} \mathrm{C}$ without shaking. $450 \mu$ lof pre-warmed S.O.C medium were added to the cells. The tube was shaken $(225 \mathrm{rpm})$ at $37^{\circ} \mathrm{C}$ for 1 hour. 30-60 $\mu \mathrm{l}$ medium from each transformation was spread on LB plate containing carbenicillin $(1 \mu \mathrm{g} / \mathrm{ml})$. The plates were incubated overnight at $37^{\circ} \mathrm{C}$.

\subsubsection{Plasmid Isolation}

About 50 clones per PCR reaction were picked from antibiotic selection plates described above. They were each inoculated to $1 \mathrm{ml} \mathrm{LB}$ medium containing carbenicillin $(1 \mu \mathrm{g} / \mathrm{ml})$ and incubated overnight at $37^{\circ} \mathrm{C}$ with vigorous shaking $(250 \mathrm{rpm})$. The bacterial cells were harvested, and cDNA-containing plasmid was purified with the Wizard SV 96 Plasmid DNA Purification System (Promega), according to the manufacture's protocol. Plasmid DNA was eluted in $100 \mu \mathrm{l}$ nuclease-free water and stored at $4^{\circ} \mathrm{C}$.

\subsubsection{CDR3 Sequencing and Analysis}

The cloned PCR products were sequenced using a T7 priming site in the cloning vector (T7, 5'-TAATACGACTCACTATAGGG). Capillary sequencing was performed by the St.Jude Hartwell Center for Biotechnology. V $\beta, J \beta$ and CDR3 nucleotide and amino acid sequences were identified with IMGT-V-Quest software (immunogenetics information system; http://imgt.cines.fr/). CDR3 charge and hydrophilicity analyses were performed with the innovagen peptide calculator (http://www.innovagen.se/).

\subsection{MOG-specificity of Transduced 4G4 Hybridomas}

\subsubsection{Vector Construction}

The orginial XhoI site of 1MOG244.2 $\alpha \beta$ MSCV-IRES-CFP vector (Alli et al., 2008 ) was deleted by DNA Polymerase I large (Klenow) fragment (Promega). A new XhoI and BamHI sites were created flanking the CDR3 $\beta$ region without altering amino acid sequence with the Quick change site directed mutagenesis kit (Stratagene)

(Figure 2-3). To generate selected Treg and Tconv TCR constructs, the CDR3 $\beta$ region identified during repertoire analyses was PCR amplified with primers incorporating an endogenous V $\beta 8.2$ BamHI site and inserting a XhoI restriction site within the $\mathrm{C} \beta$ 


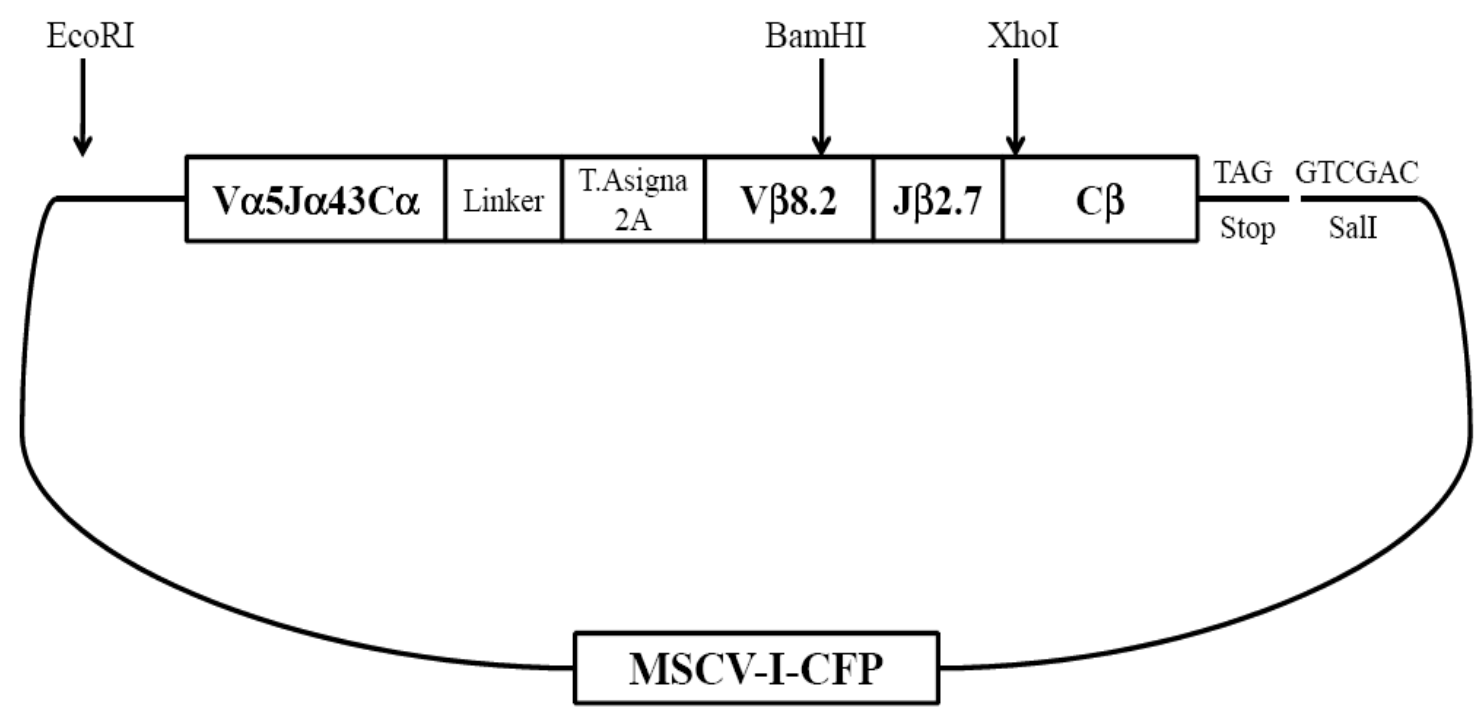

Figure 2-3. Structure of modified retroviral constructs containing new BamHI and XhoI sites. CDR $3 \beta$ region was PCR amplified with primer containing BamHI and XhoI sites, and subcloned into the MSCV-I-CFP retroviral vectors. 
(5' primer: GGTGCTGGATCCACTGAGAAAGGAGATATCCC 3' primer:

GCCTCGAGAACCGTGAGCCTGGTGC). The PCR products ( 200bp) were purified with PCR purification kit (Qiagen), and subcloned into the modified 1MOG244.2TCR $\alpha \beta$ MSCV-IRES-CFP construct, using quick T4 DNA ligase (NEB). The ligation products were chemically transformed into $\mathrm{DH} 5 \alpha^{\mathrm{TM}}$ E. coli. $60-100 \mu 1$ transformation products were spread on LB agar plates containing carbenicillin. Plates were incubated at $37^{\circ} \mathrm{C}$ overnight. $\sim 30$ clones per construct were selected and plasmid was isolated. Construct integrity was verified by DNA sequencing using a V $\beta 8.2$ primer (V $\beta 8.2,5$ 'ATGTCTAACACTGCCTTCCCTGACCC).

\subsubsection{Transformation of CD4-4G4 T-cell Hybridoma}

Ten micrograms of the TCR $\alpha \beta$ construct and $10 \mu \mathrm{g}$ of retrovirus helper DNA constructs were co-transfected into 293T-cells by calcium phosphate precipitation. At 16 $\mathrm{h}$, the cells were washed and cultured in DMEM with $10 \% \mathrm{FCS}, 100 \mathrm{U} / \mathrm{ml}$ penicillin G, $100 \mu \mathrm{g} / \mathrm{ml}$ streptomycin, $292 \mu \mathrm{g} / \mathrm{ml}$ L-glutamine (Invitrogen Life Technologies) for $48 \mathrm{~h}$. Supernatant was collected twice daily and used to infect GP+E86 retroviral producer cells in the presence of $6 \mu \mathrm{g} / \mathrm{ml}$ polybrene (hexadimethrine bromide; Sigma-Aldrich). Transduced GP+E86 cells were expanded in culture, and supernatant was used to infect murine surface TCR-deficient 4G4.CD4 T hybridoma cells (gift of Dr.Janeway, Yale University, New Haven, CT).

Murine surface TCR-deficient 4G4 hybridoma cells were first transduced with mouse CD4-expressing retrovirus (MSCV-CD4-I-GFP), to produce 4G4.CD4 cells (Alli et al., 2008; Udyavar et al., 2009). To transduce the different TCR constructs, $10^{6}$ 4G4.CD4 cells were harvested and resuspended in $6-10 \mathrm{ml}$ retroviral supernatant. The cell suspension was centrifuged at $1800 \mathrm{rpm}, 8^{\circ} \mathrm{C}$ for 90 minutes, in the presence of $6 \mu \mathrm{g} / \mathrm{ml}$ polybrene. The infected cells were then cultured in complete Click's medium. The transduced cells were flow cytometrically sorted twice for the $\mathrm{GFP}^{\text {high }} \mathrm{TCR}^{+}$ population and expanded. The purity of the $\mathrm{GFP}^{\text {high }} \mathrm{TCR}^{+} 4 \mathrm{G} 4$ cells was confirmed by flow cytometry (BD FACSCalibur).

\subsubsection{Cytokine (IL-2) Analysis by Enzyme-linked Immunosorbent Assay (ELISA)}

Cytokine sandwich ELISA is sensitive enzyme immunoassay that can specifically detect and quantitate the concentration of soluble cytokine proteins. The transduced 4G4.CD4 cells $\left(1 \times 10^{5}\right)$ were cultured in the presence of $3 \times 10^{5}$ APCs. In one experiment, they were stimulated with 10 -fold titrations of $\mathrm{MOG}_{35-55}(100 \mu \mathrm{g} / \mathrm{ml}, 10 \mu \mathrm{g} / \mathrm{ml}, 1 \mu \mathrm{g} / \mathrm{ml}$, $0.1 \mu \mathrm{g} / \mathrm{ml}$ and $0 \mu \mathrm{g} / \mathrm{ml})$. In the other experiment, cells were cultured with $100 \mu \mathrm{g} / \mathrm{ml}$ $\mathrm{MOG}_{35-55}$ or the indicated alanine-substituted peptides. $100 \mu$ of supernatant was collected from the cultures 24 hours after stimulation. To measure the IL-2 concentration, purified anti-IL-2 capture antibody (clone MQ1-17H12) was diluted to $2 \mu \mathrm{g} / \mathrm{ml}$ in binding solution. $50 \mu 1$ of the diluted antibody was added per well of a 96-well ELISA plate (Nunc Maxisorb) and incubated at $4{ }^{\circ} \mathrm{C}$ overnight. Capture antibody was then removed, 
and non-specific binding was blocked by adding $200 \mu 1$ of blocking buffer per well (BD Bioscience), and incubated at RT for $2 \mathrm{hrs}$. After wash with PBS/Tween (PBS and $0.1 \%$ Tweens) for 3 times, culture supernatants were added to the coated plate, $100 \mu \mathrm{l}$ per well. A series of nine two-fold dilutions of recombination mouse IL-2 standard (from $5000 \mathrm{pg} / \mathrm{ml}$ to $19.5 \mathrm{pg} / \mathrm{ml}$ ) was added to same wells. The plate was incubated at $4^{\circ} \mathrm{C}$ overnight. After washing away unbound material with PBS/Tweens, $100 \mu 1$ diluted biotinylated anti-IL-2 $(1 \mu \mathrm{g} / \mathrm{ml})$ was added and incubated room temperature for one hour. Again after washing with PBS/Tween for five times, $100 \mu$ l diluted peroxidase labeled anti-biotin (Vector laboratories, Burlingame, CA) was added and incubated for 30 minutes. The plate was washed for eight times and ABTS substrate solution with hydrogen peroxide was added. After color developed, the OD was read by a microplate reader (Bio-Rad) setting to $405 \mathrm{~nm}$.

\subsection{Generation of TCR-retrogenic Mixed Chimeras}

Retroviral constructs incorporating the TCR $\alpha \beta$ from MOG-specific TCRs were generated and transduced into GP+E86 producer cells as described above. These

transduced producer cells were used to infect HPCs from B6.129S7-Rag $1^{\text {tm1Mom }} / \mathrm{J}$. The transduction efficiency was evaluated by flow cytometry for CFP. The HPCs were mixed at a 1:1 or 1:10 ratio with untransduced, T-cell depleted CD45.1 congenic B6.SJLPtprc ${ }^{a}$ Pep $3^{b}$.BoyJ-derived bone marrow, and injected i.v into lethally irradiated (1100Rad) B6.SJL-Ptprc ${ }^{a}$ Pep $3^{b}$.BoyJ mice.

\subsection{Flow Cytometric Analysis of TCR-retrogenic Mixed Chimeras}

Eight weeks after transplantation, splenocytes and thymocytes were isolated. Entire splenocytes were stained with a mix of antibodies specific for CD4 (clone L3T4), TCR (clone H57-597) and CD45.2 (clone 104). Entire thymocytes were stained with the mix of antibodies specific for CD4, CD8, and CD45.2. After washing, the cells were fixed and permeabilized by $1 \mathrm{ml}$ Fixation/Permeabilization working solution for 30 minutes (eBioscience). Cells were then stained with PE conjugated anti-Foxp3 (clone FJK-16s, eBioscience), and analyzed by flow cytometry. 


\section{CHAPTER 3. GENERATION OF 1MOG244.2TCR $\alpha$ RETROGENIC MICE}

\subsection{Introduction}

Immune homeostasis is maintained partially by Treg cells that dominantly control potential hazardous self-reactive T-cells in the periphery. Their suppressive features and direct inhibition of autoimmune diseases attracted a decade of active investigation into their biology. TCR repertoire analyses shed light on the origin of Treg and their relationship with Tconv cells. This chapter describes the development of a mouse model that is not only susceptible for EAE but also facilitated the performance of Treg repertoire analysis.

The diversity of the mature T-cell repertoire is generated by the rearrangement of various gene segments and addition of nucleotides not present in germline to form the $\alpha$ and $\beta$ chain of the TCR, and the random paring of $\alpha$ and $\beta$ chains. In theory, more than $10^{15}$ different possible $\alpha \beta$ TCR may form in mice (Davis and Bjorkman, 1988). But in reality, most (about 95\%) of thymocytes die during the development processes as they fail either positive or negative selection. Only 5\% survive to become mature T-cells. Casrouge et al. has estimated that the actual mouse naïve T-splenocytes repertoire contains $2 \times 10^{6}$ unique clones by the immunoscope technique. This figure, although orders of magnitude smaller than the maximum theoretical diversity $\left(10^{15}\right)$, is still large enough to maintain a high functional level of diversity (Casrouge et al., 2000).

The ideal way to analyze TCR repertoire would be identify both $\alpha$ and $\beta$ chains of each T-cell. However, technical limitations make it impractical to perform this on a large scale. Most current TCR repertoire analyses rely on the use of transgenic mice in which one chain of the TCR is fixed (Hsieh et al., 2004; Malherbe et al., 2004; Wong et al., 2007b; Zehn and Bevan, 2006). The fixed $\alpha$ or $\beta$ chain limits the repertoire variation to the alternative chain. This technique not only simplifies the complicated system, but also, if the fixed chain has a propensity for auto-reactivity, will result in larger than normal populations of self-reactive cells, thereby facilitating analyses of the self-reactive repertoire. We used lethally irradiated mice reconstituted with bone marrow progenitor cells transduced with a retrovirus incorporating a rearranged TCR $\alpha$ chain, so called retrogenic mice (Holst et al., 2006a). The advantages of the retrogenic system are rapid development (2months) and that they allow analysis of mice expressing different TCR $\alpha$ simultaneously. The major alternative TCR transgenic mice are more costly and time consuming to create, and surveying multiple TCR would be difficult. On the other hand, fewer T-cells develop in retrogenic mice than transgenic mice and they cannot propagate by breeding. Here we developed a retrogenic model of MOG-EAE in which the TCR $\alpha$ chain locus is fixed by the enforced retrogenic expression of a TCR $\alpha$ from a MOG-specific T-cell. 


\subsection{Results}

\subsubsection{Retrogenic Mice Expressing a Single TCR V $\alpha$ Chain}

To generate a retrogenic mouse model susceptible to EAE, we first generated several different strains of retrogenic mice, each incorporating a different $\alpha$ chain. Briefly, we bred Ca KO mice (Mombaerts et al., 1993) with GFP-FoxP3 KI mice (Fontenot et al., 2005b), and transduced the bone marrow of the offspring with retroviral constructs incorporating the $\alpha$ chain from 1MOG244.2, 2MOG10, 1MOG9, and 1MOG213 MOG-specific TCRs. C $\alpha$ KO mice do not express any $\alpha$ chain. That ensures the bone marrow cells only contain the rearranged transduced $\alpha$ chain. Because $\mathrm{C} \alpha \mathrm{KO}$ mice have normal RAG-recombinase and an intact TCR of $\beta$ genome, the complete genome of $\beta$ chains, the engrafted bone marrow progenitor cells will complete $\beta$ chain rearrangement from genomic $\mathrm{V}, \mathrm{D}, \mathrm{J}$ segments and develop into $\mathrm{T}$-cells with a diverse repertoire. In addition, the GFP-FoxP3 transgene allows the rapid identification of regulatory T-cells $\left(\mathrm{CD}^{+} \mathrm{GFP}^{+}\right)$and conventional T-cells $\left(\mathrm{CD}^{+} \mathrm{GFP}^{-}\right)$by GFP florescence.

6 weeks post hematopoietic progenitor cell (HPC) transplantation, the peripheral blood were collected and stained with antibodies specific for CD4 and TCR $\beta$. Engraftment was verified by assessing for the presence of $\mathrm{CD}^{+} \mathrm{TCR}^{+}$in gated lymphocytes, and this ranged from $1 \%$ to $5 \%$. Mice were challenged with $\mathrm{MOG}_{35-55}$ peptide in CFA, as described in Chapter 2. The retrogenic mice bearing the different specific TCR $\alpha$ chains show inducible EAE, but the severity of disease was strongly influenced by the introduced $\alpha$ chain. Only 1MOG244.2 $\alpha$ retrogenic mice developed EAE with high penetrance in response to $\mathrm{MOG}_{35-55}$ immunization, with $>90 \%$ of mice developing typical disease symptoms (Figure 3-1B). Mice expressing other TCR $\alpha$ developed more mild disease (Figure 3-1A). Hence, we chose 1MOG244.2TCR $\alpha$ retrogenic mice as a mouse model to further evaluate the TCR repertoire.

\subsubsection{Phenotype of 1MOG244.2TCR $\alpha$ Retrogenic Mice}

At approximately eight weeks post-HPC transfer, the retrogenic mice showed good lymphoid engraftment. Thymocyte subsets showed no significant difference in absolute cell number, percent of DN, DP, $\mathrm{CD}^{+} \mathrm{SP}$ and $\mathrm{CD} 8^{+} \mathrm{SP}$ subsets compared with $\mathrm{C} 57 \mathrm{BL} / 6$ mice, though they trended toward increased double negative $(\mathrm{DN})$ cells $(7.0 \% \pm$ $6.8 \% 1 \mathrm{MOG} 244.2 \alpha$ versus $3.0 \% \pm 1.3 \% \mathrm{C} 57 \mathrm{BL} / 6)$ and decreased CD4 ${ }^{+}$SP cells $(1.7 \% \pm$ $1.8 \%$ versus $7.0 \% \pm 3.8 \%$ ) (Figure $3-2 \mathrm{~A}$ ). Specific analyses of DN3 and DN4 cells subpopulation revealed no statistical difference between retrogenic mice and C57BL/6 mice Retrogenic mice showed a slightly decreased percent of DN4 subsets among total DN cells $(47.93 \% \pm 5.868 \%$ 1MOG $244.2 \alpha$ versus $55.33 \% \pm 6.886 \%$ C57BL/6) (Figure $3-2 \mathrm{~B}-\mathrm{D})$, though not statistically significant. The DN4 population identified in retrogenic mice demonstrated that a substantial number of DN3 cells pass through $\beta$-selection checkpoint while expressing the retrogenic TCR $\alpha$ chain. This is significant as some 

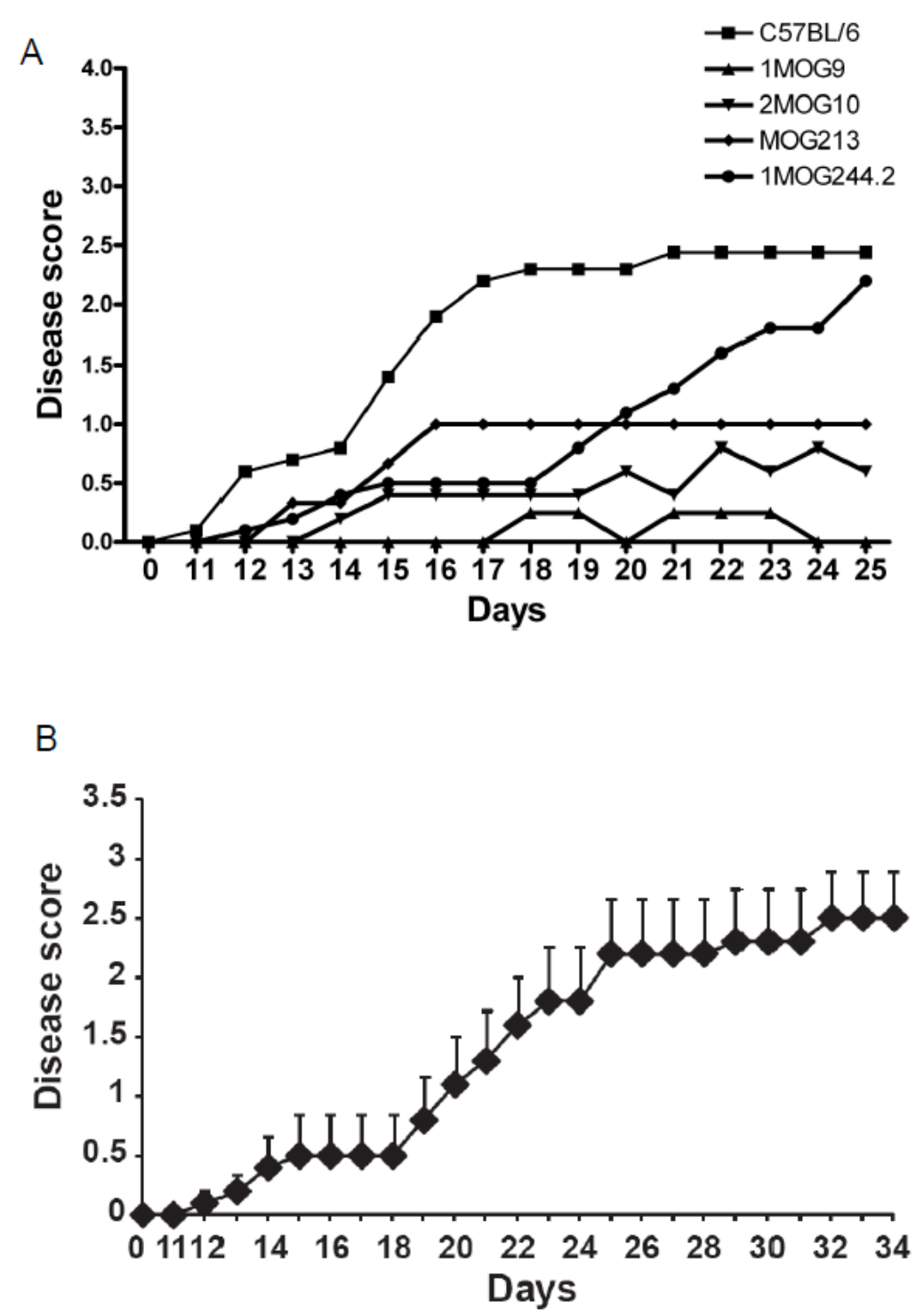

Figure 3-1. EAE induction in retrogenic mice. (A) 1MOG244.2, 2MOG10, 1MOG9, and $1 \mathrm{MOG} 213 \mathrm{TCR} \alpha$ retrogenic and wild-type mice $(\mathrm{n}=4$ or 5) were immunized with $\mathrm{MOG}_{35-55}$. EAE was clinically monitored. Mean clinical score is plotted. (B) EAE was induced on 1MOG244.2 $\alpha$ mice $(n=10)$. Ten of 10 mice developed disease symtoms with a mean \pm 1 s.d. maximal score of $2.6 \pm 1.4$ (range 1-5). Plot shows mean clinical score \pm 1 s.e.m. 


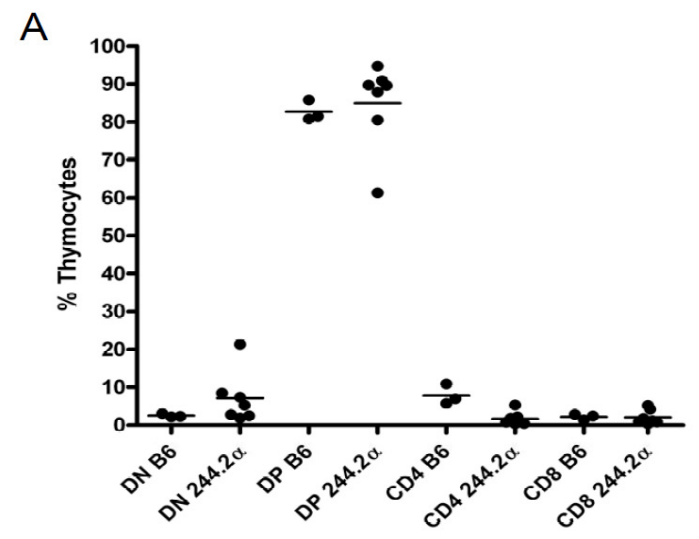

B
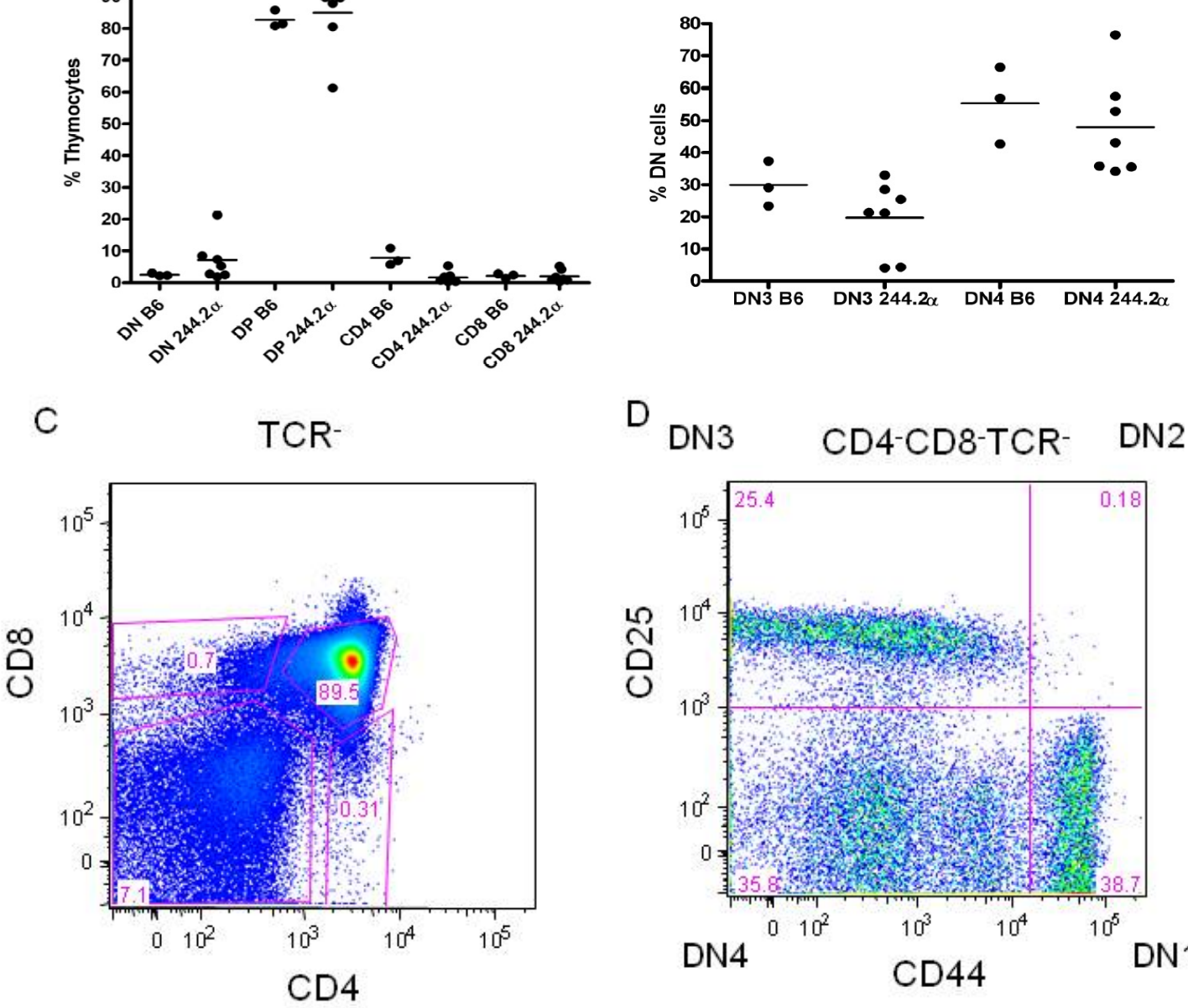

DN4

CD44

DN1

Figure 3-2. Thymocyte reconstitution in retrogenic mice. $1 \mathrm{MOG} 244.2 \alpha$ retrogenic mice were sacrificed $\sim 8$ weeks after HPC transfer, and thymocytes were isolated and quantitatively analyzed for the indicated cell subsets. (A) Percent of DN, DP, or CD4 ${ }^{+}$or $\mathrm{CD} 8{ }^{+}$SP T-cells among 1 MOG244.2 $\alpha$ or simultaneously analyzed C57BL/6 thymocytes is shown. (B) Percent of DN3 or DN4 cells among CD4 CD8 ${ }^{-} \mathrm{TCR}^{-}$thymocytes. (C and D) Flow cytometric analysis shows DN3 and DN4 cells development in the CD4 ${ }^{-} \mathrm{CD}^{-}$ $\mathrm{TCR}^{-}$gated populations. 
reports have shown a decreased DN3 to DN4 transition in TCR $\alpha$ transgenic mice (Baldwin et al., 2005; Borowski et al., 2004; Huang and Kanagawa, 2004; Lacorazza et al., 2001).

Analyses of the periphery showed a mean of $83 \pm 6 \times 10^{6}$ total splenocytes in retrogenic mice, primarily B-cells (Figure 3-3C). Engrafted T-lymphocytes were decreased compared to wild-type C57BL/6 mice (data available at www.jax.org), and comprised $13.8 \pm 4.9 \%$ of lymphocytes in the spleen and $9.7 \pm 2.9 \%$ in the lymph nodes (LN) (Figure 3-3A and B). The CD4/CD8 ratio was elevated, $6.2 \pm 1.3$ in the lymph nodes (LN) and $10.0 \pm 2.7$ in the spleen compared with $\sim 1.6$ in C57BL/6 mice (Figure 3-3D), indicating that the $1 \mathrm{MOG} 244.2 \mathrm{TCR} \alpha$ developmentally skewed cells to the CD4 lineage. $\mathrm{CD}^{+} \mathrm{T}$-cells expressed the GFP-Foxp3 transgene in proportions comparable to that observed in wild-type mouse strains, $12.8 \pm 2.2 \%$ in LN and $7.5 \pm 2.4 \%$ in spleen (Figure 3-3A and B). Therefore mice expressing the 1MOG244.2 $\alpha$ transgene develop a repertoire that incorporates normal proportions of $\mathrm{CD}^{+}$Treg and Tconv cells and they are susceptible to EAE, though showing decreased representation of $\mathrm{CD} 8^{+} \mathrm{T}$-cells.

\subsubsection{VßUse in 1MOG244.2TCR $\alpha$ Splenic T-lymphocytes}

We first analyzed peripheral TCR repertoire in unimmunized 1MOG244.2 $\alpha$ mice by examining $\mathrm{V} \beta$ chain usage by monoclonal antibody (MAbs) staining (Figure 3-4). These mice, with a fixed V $\alpha 5-\mathrm{J} \alpha 43$ chain, showed different V $\beta$ use compared with wildtype C57BL/6 mice. A substantially increased usage of $\mathrm{V} \beta 14$ was seen, $15.2 \% \pm 4.1 \%$ in retrogenic mice versus $6.49 \% \pm 0.48 \%$ in wild-type $(p=0.0128)$. Significantly fewer $\mathrm{V} \beta 3(\mathrm{p}=0.0018), \mathrm{V} \beta 4(\mathrm{p}=0.0348)$, and V $\beta 8.3(\mathrm{p}=0.003) \mathrm{T}$-cells were seen, potentially due to the compensation for the increased V $\beta 14$ use. However, V $\beta 8.1 / 8.2$ was the most frequently used TCR among retrogenic mice, and wild-type mice showed a similar percentage.

\subsubsection{Increased TCR VB8.2 Use in CNS-infiltrating 1MOG244.2 $\alpha$ T-cells}

As a low resolution screen for EAE-associated alterations in the 1MOG244.2 $\alpha$ TCR repertoire, we analyzed $\mathrm{V} \beta$ chain use by staining splenic or CNS CD ${ }^{+}$GFP-Foxp3and GFP-Foxp3 ${ }^{+}$cells with V $\beta$-specific Abs. A significant increase in V $\beta 8.1 / 8.2^{+}$use in CNS-infiltrating Tconv was seen $(p=0.018)$ compared with paired splenic Tconv or Tconv from unimmunized controls (Figure 3-5A). This is consistent with the documented over-representation of this receptor among $\mathrm{MOG}_{35-55} / \mathrm{Ab}$-specific $\mathrm{T}_{\text {eff }}$ cells (Mendel et al., 1995; Mendel Kerlero de Rosbo and Ben-Nun, 1996). A trend toward increasing V $\beta 8.1 / 8.2$ TCR use was also seen among CNS Treg (Figure 3-5B), though this did not achieve statistical significance. A decrease in V $\beta 8.3$ TCR among Treg cells $(\mathrm{p}=$ 0.048 ) was also seen, possibly due to compensation for the increased V $\beta 8.1 / 8.2$ usage. Therefore 1MOG244.2 $\alpha$ mice express a TCR repertoire that is modified by EAE induction. Further, V $\beta 8.1 / 8.2$-expressing T-cells are concentrated in the CNS during EAE, implying increased MOG-reactivity in the infiltrating T-cell population. 
A

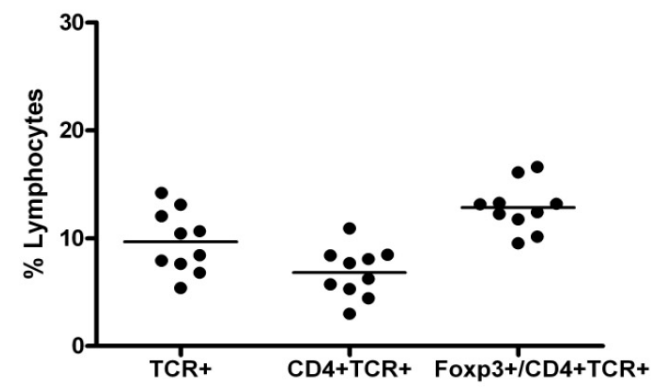

C

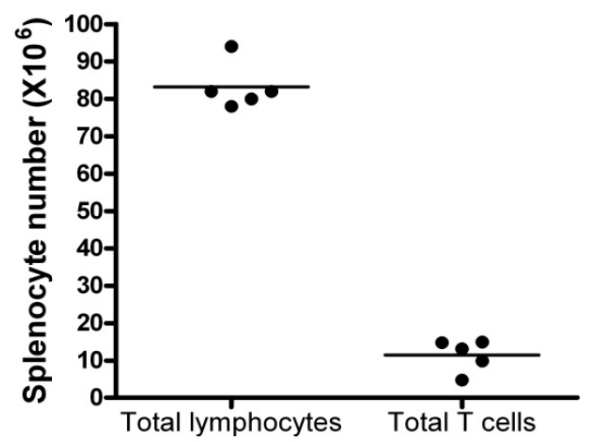

$B$
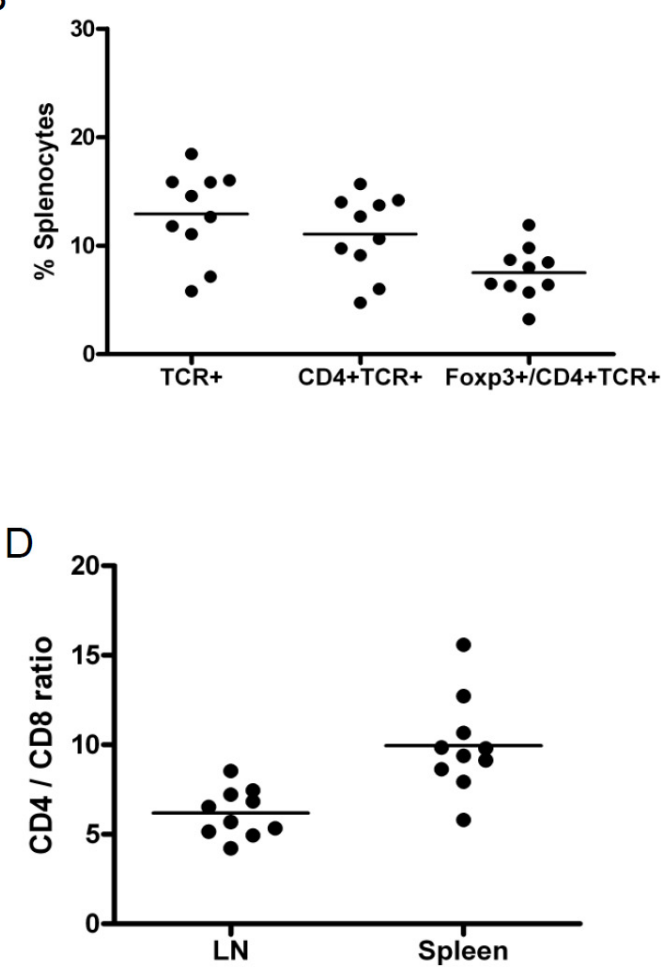

Figure 3-3. Lymphoid reconstitution of retrogenic mice. 1MOG244.2 $\alpha$ retrogenic mice were sacrificed $\sim 8$ weeks after HPC transfer. Spleen and LN cells were isolated and quantitatively analyzed for the indicated cell subsets. Percent LN (A) and splenic (B) reconstitution with total T-lymphocytes $\left(\mathrm{TCR}^{+}\right)$and $\mathrm{CD}^{+} \mathrm{T}$-lymphocytes $\left(\mathrm{CD}^{+} \mathrm{TCR}^{+}\right)$, and fractional engraftment with $\mathrm{CD}^{+} \mathrm{TCR}^{+}$GFP-Foxp ${ }^{+}$T-lymphocytes $\left(\mathrm{Foxp}^{+} / \mathrm{CD}^{+} \mathrm{TCR}^{+}\right)$are plotted for individual mice. Means are indicated by the horizontal bar. The remaining lymphocytes were composed predominantly of B-cells. (C) Absolute number of total splenocytes and $\mathrm{TCR}^{+}$splenocytes is plotted. (D) The ratio of $\mathrm{CD}^{+} / \mathrm{CD}^{+} \mathrm{T}$-cells is plotted. 


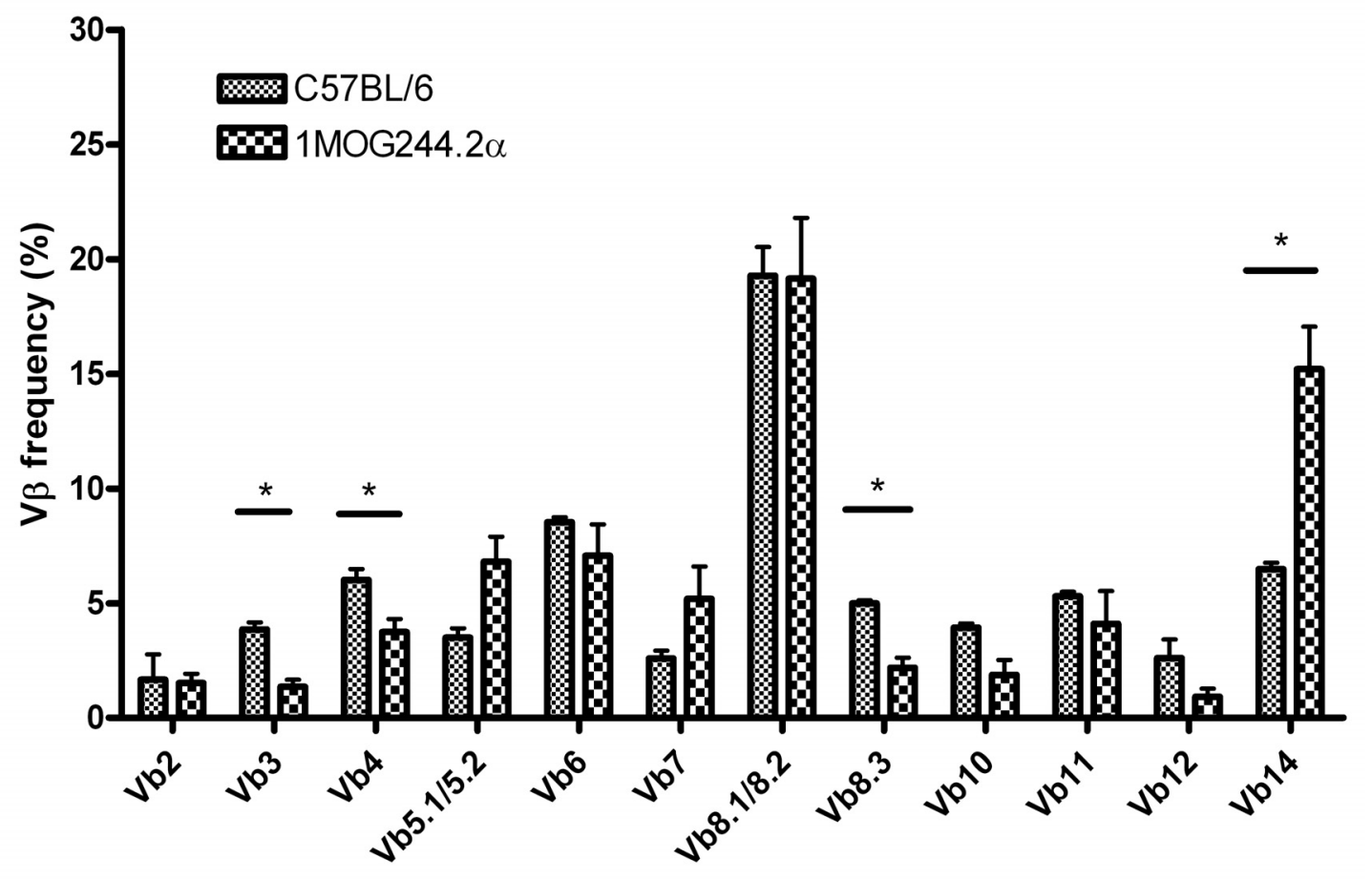

Figure 3-4. V $\beta$ use by splenic Tconv in 1MOG244.2 $\alpha$ and C57BL/6 mice. 8 weeks post transfer of HPCs, 1MOG244.2 $\alpha(\mathrm{n}=5)$ were sacrificed and $\mathrm{CD} 4^{+} \mathrm{T}$-cells were flow cytometrically analyzed using a panel of $V \beta$-specific antibodies. C57BL/6 mice $(n=4)$ were analyzed simultaneously. Significant differences between wild-type and retrogenic mice were seen. ${ }^{*}, \mathrm{p}<0.05$. 

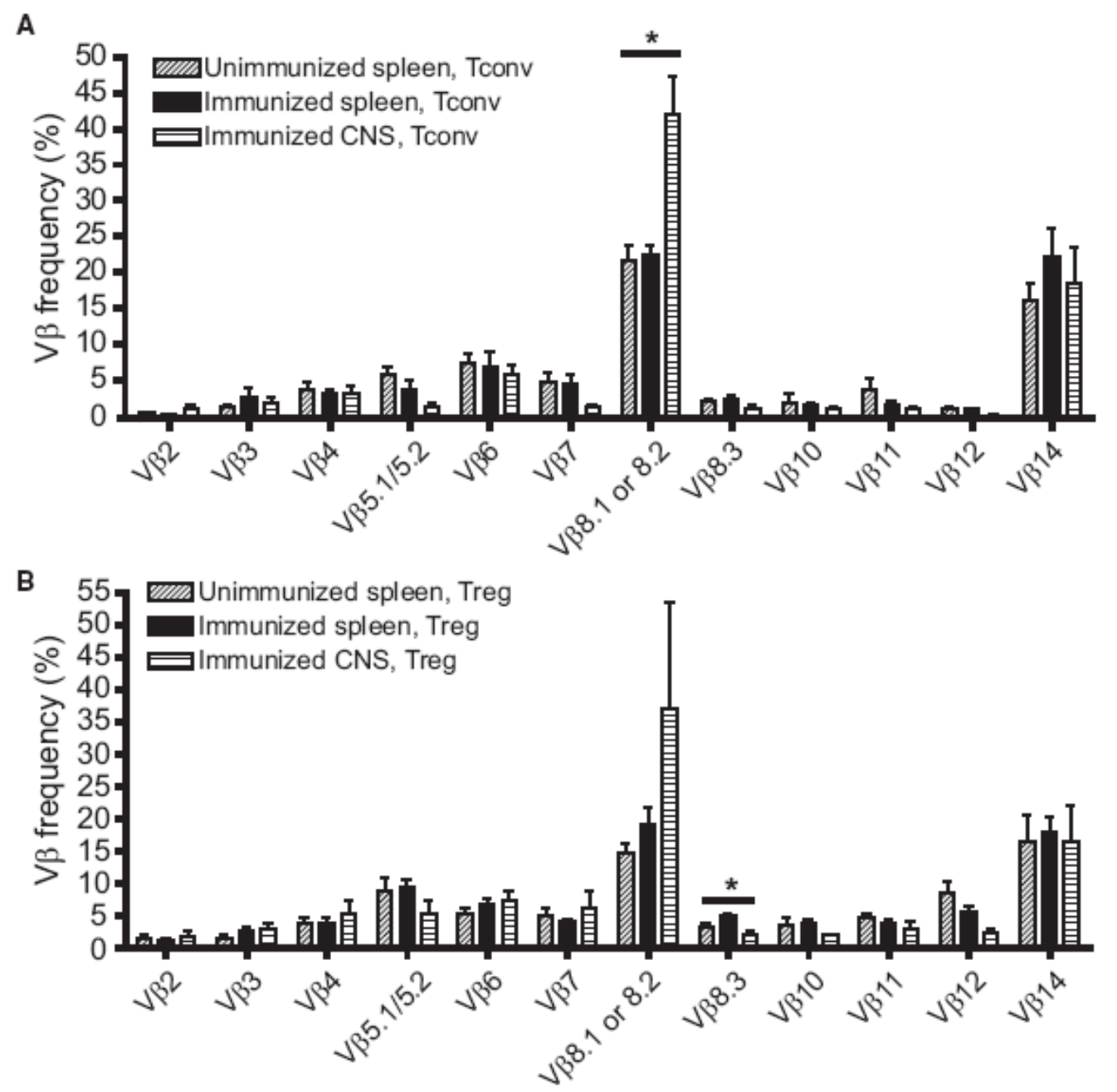

Figure 3-5. V $\beta$ use by retrogenic Treg and Tconv in pre-immune splenocytes and splenocytes and CNS-infiltrating cells from mice with EAE. Preimmune retrogenic mice or mice 17 days after EAE induction were sacrificed and $\mathrm{CD} 4^{+} \mathrm{T}$-lymphocytes flow cytometrically analyzed using a panel of V $\beta$-specific antibodies. Comparisons were made between Tconv (A) and Treg (B) cells from unimmunized and immunized splenocytes and CNS T-cells from mice with EAE. Data show mean +1 SEM. *, $p<$ 0.05 among group by Hochberg-adjusted 1-way ANOVA. 


\subsection{Summary}

TCR repertoire analysis has been used to determine the origins of Treg and their relationship with Tconv cells. This type of study is difficult to perform directly on the naive repertoire due to its high diversity. To circumvent this problem, we developed retrogenic mice expressing a retrovirally transfected TCR $\alpha$ chain. Briefly, $\alpha$ chain isolated from MOG-specific TCR was subcloned into MSCV-IRES-CFP vector. Bone marrow stem cells from Tcr $\alpha^{-/-}$GFP-Foxp3 mice were retrovirally transfected with $\alpha$ chain, and then transplanted into irradiated Tcr $\alpha^{-/-}$mice. Several different TCR $\alpha$ retrogenic mice were generated and challenged with $\mathrm{MOG}_{35-55}$ in CFA. 1MOG244.2 $\alpha$ mice showed similar disease severity as C57BL/6 mice, though the mice experienced later disease onset than wild-type mice.

Analyses of $1 \mathrm{MOG} 244.2 \alpha$ thymocytes revealed no significant differences compared with C57BL/6 mice. The DN to DP transition was efficient, and the percent of DN3 and DN4 among DN population did not show significant differences to wild-type mice. This suggests that the premature TCR $\alpha$ expression which in some models leads to diminish p $\alpha$ signaling does not alter the balance of different thymocyte subsets in $1 \mathrm{MOG} 244.2 \alpha$ mice. Further analyses of peripheral lymphocytes showed a decreased percent of T-cells, which potentially reflects the effects of transplantation into the $\mathrm{TCR} \alpha^{-/-}$mice. The ratio of CD4/CD8 is elevated five-fold versus wily type splenocytes. This suggests that the enforced $\alpha$ chain expression impacts the peripheral repertoire to some extent, favoring the $\mathrm{CD}^{+}$lineage commitment or expansion. More importantly, the proportion of Treg cells among $\mathrm{CD} 4{ }^{+} \mathrm{T}$-cells is normal. Treg lineage assignment in 1MOG244.2 $\alpha$ mice does not appear to be altered by the fixed $\alpha$ chain.

$\mathrm{V} \beta$ usage analysis was performed on unimmunized retrogenic and C57BL/6 mice. $\mathrm{V} \beta 8.1 / 8.2$ was the most frequently used TCR $\beta$ in both of these. However, the percent of $\mathrm{V} \beta 14$ cells was substantially increased in retrogenic mice, suggesting that the enforced $\alpha$ chain did influence on V $\beta$ selection. After EAE induction, T-cells bearing V $\beta 8.1 / 8.2$ accumulate in CNS in C57BL/6 mice, and this was similarly seen in the retrogenic mice. Overall, although some difference exist between wild-type and 1MOG244.2TCR $\alpha$ retrogenic mice, this mouse does appear suitable for the analysis of repertoires in EAE. 


\section{CHAPTER 4. TCR $\beta$ REPERTOIRE ANALYSIS OF 1MOG244.2TCR $\alpha$ RETROGENIC MICE}

\subsection{Introduction}

Treg cells can be produced in the thymus as a functionally mature, distinct T-cell population. Sakakura first showed that neonatal thymectomy of female mice at 3 days of age led to autoimmune oophoritis and sterility (Nishizuka and Sakakura, 1969).

Subsequent studies showed that other organs could be affected depending on the genetic background of the inbred mouse strain. Disease could be prevented either by thymus engraftment or by transfer of normal day 7 or adult spleen cells (Kojima et al., 1980; Kojima et al., 1976). Efforts to search for more specific markers for Treg identified the CD25 molecule (the IL-2 receptor $\alpha$ chain) as a candidate (Sakaguchi et al., 1995). Subsequently, the transcription factor Foxp3 was shown to be critical for Treg differentiation in thymus (Fontenot et al., 2003; Hori et al., 2003; Khattri et al., 2003). Over-expression of Foxp3 on naïve human $\mathrm{CD}^{+} \mathrm{T}$-cells confers suppressor function (Oswald-Richter et al., 2004; Yagi et al., 2004), and ectopic expression of a transgene encoding Foxp3 in mice (Khattri et al., 2003) or retroviral expression of Foxp3 in CD25 $\mathrm{CD}^{+}{ }^{+} \mathrm{T}$-cells results in the acquisition of suppression activity (Fontenot et al., 2003; Hori et al., 2003). Conversely, genetic disruption of Foxp3 in humans and mice leads to autoimmune disease. Foxp3 is currently the most reliable molecular marker for natural Tregs and provides clues to decipher the molecular basis of Treg development and function. Furthermore, studies showing the $\mathrm{CD} 4{ }^{+} \mathrm{CD} 25^{+} \mathrm{T}$-cells from MHC class II deficient mice do not suppress responder T-cells in vitro or in vivo, suggested that the development of Treg cells is dependent on MHC class II-positive thymic cortical epithelium (Bensinger et al., 2001).

Despite advances in our understanding of Treg development and function, we still do not know how these cells are generated and the role of TCR specificity in diverting thymocytes into the conventional or regulatory lineage. Sequence analyses of TCR $\alpha$ segments of a particular V $\alpha$ subfamily in Treg cells from TCR $\beta$-fixed transgenic mice have revealed strong similarity between the thymic and peripheral Treg TCR repertoire (Hsieh et al., 2006; Pacholczyk et al., 2006; Wong et al., 2007b), echoing the view that Treg cells are mainly generated in the thymus (Itoh et al., 1999). However, the peripheral and thymic Treg TCR repertoires were not identical, suggesting that certain TCRs are preferentially enriched or deleted in the periphery, similar to the observations with the total $\mathrm{CD}^{+}$T-cell population (Correia-Neves et al., 2001). Further analyses of the TCR sequences from fractionated Treg or Tconv cells demonstrated distinct repertoires of the two populations, although with a degree of overlap that differed depending on the experimental setting. These studies were performed with unimmunized adult mice, and the relationship between the two subsets in mice with autoimmune disease like EAE circumstance was still unclear. To clarify the TCR repertoire differences between Treg and Tconv cells in MOG-EAE, we examined J $\beta$ usage and the CDR3 $\beta$ sequence of Treg and Tconv cells from 1MOG244.2 $\alpha$ mice with or without EAE. 


\subsection{Results}

\subsubsection{TCR J $\beta$ and CDR3 Use in MOG-EAE}

To examine alterations in the Treg and Tconv repertoires at higher resolution, we sacrificed 1MOG244.2 $\alpha$ mice prior to immunization or 23-25 days after EAE induction. Spleen and, for mice with EAE, CNS cells were collected and pooled from 5-6 identically treated mice per group. Pooling was performed due to low post-isolation T-cell yields from the CNS of retrogenic mice. The cells were sorted based on CD4 and the presence or absence of GFP-Foxp3 expression. RT-PCR was performed to analyze the paired $\beta$ chains in each organ and cell type. $\mathrm{V} \beta 8.2-\mathrm{C} \beta$ or $\mathrm{V} \beta 8.2-\mathrm{J} \beta 2.7$ primers were used to amplify TCR cDNA. We established a robust system to PCR amplify TCR $\beta$ cDNA without contamination, and the same experiment was repeated twice. Five PCR reactions were performed on each cDNA to avoid PCR bias. The 400 bp DNA segments were TA cloned into pCR2.1 vector, 50-100 clones were picked and sequenced the with T7 primer. Finally, we submitted the sequences in bulk to the IMGT (immunogenetics information system; http://imgt.cines.fr/) web server for identifying the TCR gene use. Totally 4000 sequences were collected. The identical experiment was performed twice.

We initially surveyed V $\beta 8$.2-C $\beta$ sequences to determine the distribution of $\mathrm{J} \beta$ among V $\beta 8.2^{+}$TCR. In total, 1829 sequences were assessed. Twelve different $\mathrm{J} \beta$ regions were found in these TCR sequences. J $\beta$ used by Treg and Tconv cells showed no significant difference in either spleen or CNS (Figure 4-1C and D). This hinted that Treg cells have a diverse V $\beta 8.2^{+}$TCR repertoire as Tconv cells, and this diversity was not altered by autoimmunity circumstances. Proportions of $\mathrm{J} \beta$ used by either the Foxp $3^{+}$or Foxp3- populations showed no significant differences between the CNS and periphery in mice with EAE ( $p>0.05$, Figure 4-1A and B). Comparison of pre- and post-immune splenocytes also failed to identify changes in J $\beta$ use among Foxp $3^{+}$T-cells and showed a significant difference in Foxp3 ${ }^{-}$cells only for a single $J \beta, J \beta 2.4$ (6.2 $\pm 0.5 \%$ pre versus $14.1 \pm 0.5 \%$ post $\mathrm{p}=0.012$ ). This indicates preserved diversity in the $\mathrm{V} \beta 8.2^{+}$population with EAE induction, especially when comparing the CNS-infiltrating and splenic T-cell populations of diseased mice.

We next surveyed the more circumscribed V $\beta 8.2-\mathrm{J} \beta 2.7$ repertoire. V $\beta 8.2$ was selected due to the predominate expression of V $\beta 8.2$ among MOG-specific T-cells in $\mathrm{H}$ $2^{\mathrm{b}}$ mice (Mendel Kerlero de Rosbo and Ben-Nun, 1996) and J $\beta 2.7$ due to its use in the original V $\beta 8.2^{+} \mathrm{J} \beta 2.7^{+} \mathrm{TCR} \beta$ chain partner for the 1MOG244.2 $\alpha$ TCR (Alli et al., 2008). Despite the theoretical potential for formation of $>10^{6}$ unique TCR sequences with this $\mathrm{V} \beta-\mathrm{J} \beta$ constraint, we therefore knew that $\mathrm{V} \beta 8.2^{+} \mathrm{J} \beta 2.7^{+} \mathrm{TCR} \beta$ had the potential to pair with the 1MOG244.2 $\alpha$ to form MOG-specific TCR. An estimated 95\% confidence interval for the upper limit of the maximum frequency $\left(\varphi_{\max }\right)$ of unobserved TCR CDR3 sequences for individual cell types/samples in different experiments ranged from 1.4 to $3.1 \%$ for different samples and cell types (Table 4-1). The $\varphi_{\max }$ confidence interval (CI) refers to the upper frequency of a CDR3 sequence in the V-J analysis for which there is 
Figure 4-1. $\mathbf{J} \boldsymbol{\beta}$ used in Tconv and Treg cells. $C D 4^{+} \mathrm{T}$-cells from retrogenic mice, preimmune or EAE induction, were flow cytometrically sorted into CD ${ }^{+} \mathrm{Foxp}^{-}{ }^{-}$and $\mathrm{CD} 4{ }^{+} \mathrm{Foxp}^{+}$populations. After a few steps of RT-PCR, TA clone and sequencing, $\mathrm{CDR} 3 \mathrm{~b}$ regions were identified through IMGT. Comparisons were made between Tconv (A) and Treg (B) cells from unimmunized and immunized splenocytes and CNS T-cells from mice with EAE. Comparisons were also made between Tconv and Treg cells in immunized CNS T-cells (C) and in immunized splenocytes (D). $* \mathrm{P}<0.05$. 
A
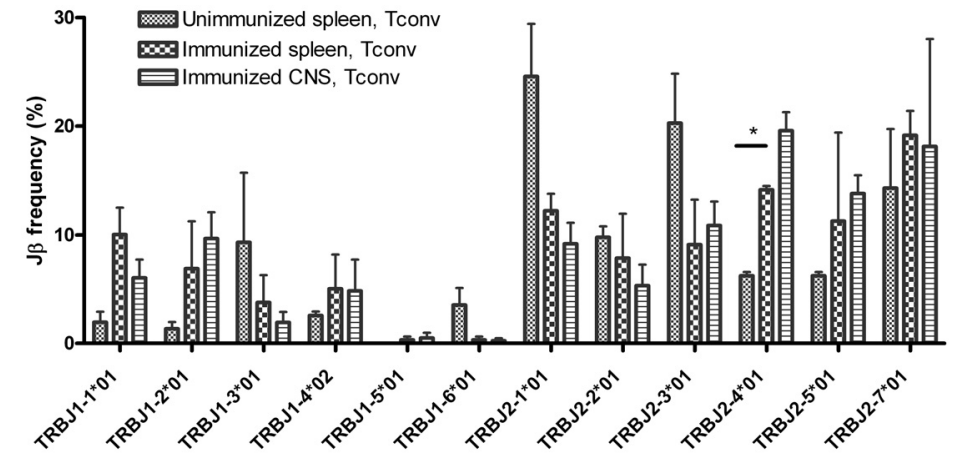

B

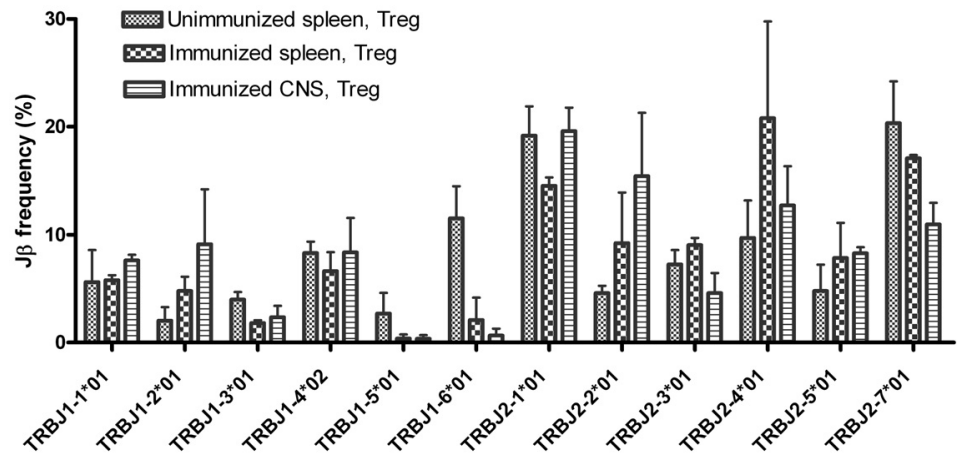

C
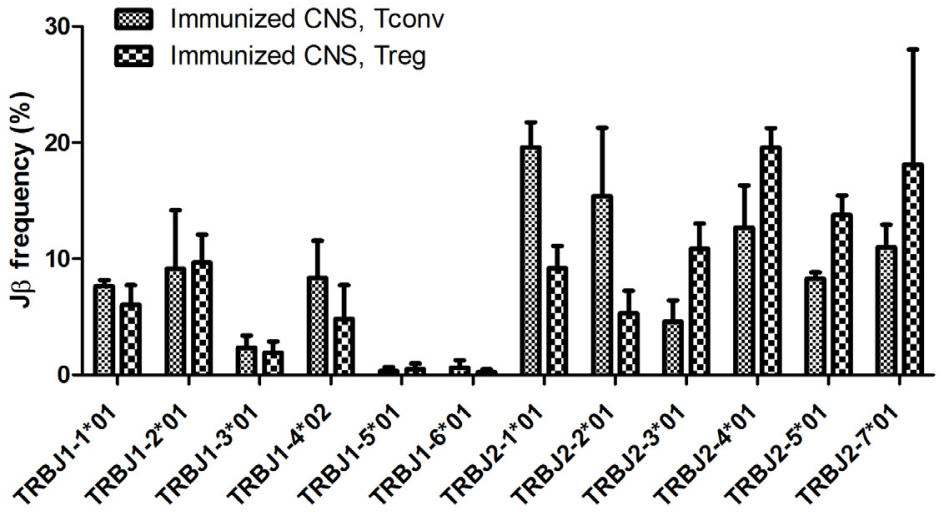

D

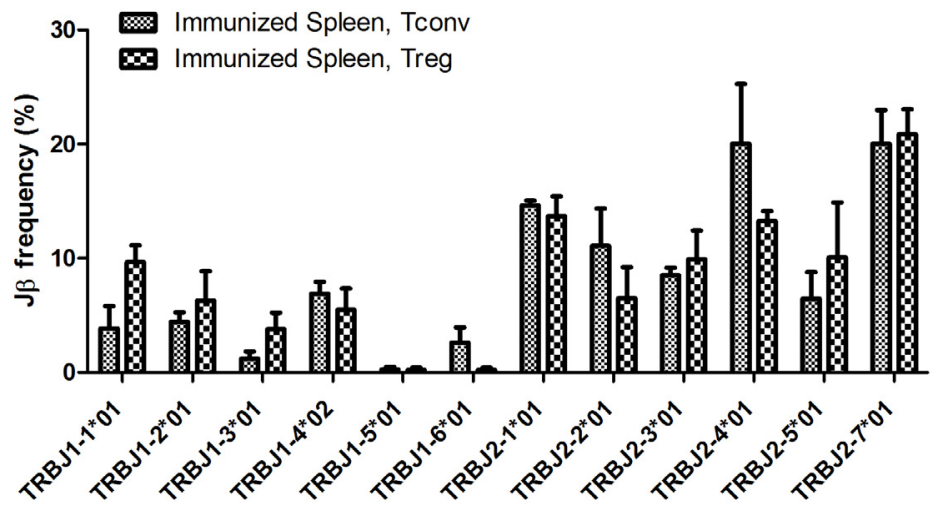


Table 4-1. Isolation of TCR V $\beta$ sequences from retrogenic mice.

\begin{tabular}{|c|c|c|c|c|c|c|}
\hline \multirow{2}{*}{ Experiment } & \multirow{2}{*}{$\begin{array}{l}\text { Disease } \\
\text { scores }\end{array}$} & \multirow{2}{*}{ T-cell type } & \multirow{2}{*}{ Sequence } & \multicolumn{2}{|c|}{$\begin{array}{c}\text { Isolated } \\
\text { sequences }\end{array}$} & \multirow{2}{*}{$\begin{array}{l}\text { V-J фmax } \\
95 \% \mathrm{CI}\end{array}$} \\
\hline & & & & $\mathrm{V}-\mathrm{C} \beta$ & V-J $\beta$ & \\
\hline \multirow[t]{4}{*}{$\begin{array}{l}1 \text { (Pre- } \\
\text { immune) }\end{array}$} & \multirow[t]{4}{*}{ NA } & \multirow{2}{*}{ Spleen Foxp3- } & Total & 137 & 155 & \\
\hline & & & Unique & 131 & 55 & $2.25 \%$ \\
\hline & & \multirow{2}{*}{ Spleen Foxp $3^{+}$} & Total & 152 & 183 & \\
\hline & & & Unique & 150 & 64 & $1.91 \%$ \\
\hline \multirow[t]{4}{*}{$\begin{array}{l}2 \text { (Pre- } \\
\text { immune) }\end{array}$} & \multirow[t]{4}{*}{ NA } & \multirow{2}{*}{ Spleen Foxp3- } & Total & 102 & 161 & \\
\hline & & & Unique & 97 & 54 & $2.17 \%$ \\
\hline & & \multirow{2}{*}{ Spleen Foxp $3^{+}$} & Total & 128 & 123 & \\
\hline & & & Unique & 124 & 62 & $2.85 \%$ \\
\hline \multirow[t]{8}{*}{$\begin{array}{l}3 \text { (EAE, Day } \\
23)\end{array}$} & \multirow[t]{8}{*}{$\begin{array}{l}1,2,3,3 \\
4\end{array}$} & \multirow{2}{*}{ Spleen Foxp3- } & Total & 159 & 167 & \\
\hline & & & Unique & 97 & 52 & $2.09 \%$ \\
\hline & & \multirow{2}{*}{ Spleen Foxp $3^{+}$} & Total & 131 & 141 & \\
\hline & & & Unique & 57 & 46 & $2.48 \%$ \\
\hline & & \multirow{2}{*}{ CNS Foxp3- } & Total & 207 & 256 & \\
\hline & & & Unique & 90 & 42 & $1.36 \%$ \\
\hline & & \multirow{2}{*}{ CNS Foxp $3^{+}$} & Total & 147 & 226 & \\
\hline & & & Unique & 88 & 59 & $1.54 \%$ \\
\hline \multirow[t]{8}{*}{$\begin{array}{l}4 \text { (EAE, Day } \\
25)\end{array}$} & \multirow[t]{8}{*}{$\begin{array}{l}1,1,1,2, \\
2,2\end{array}$} & \multirow{2}{*}{ Spleen Foxp3- } & Total & 160 & 208 & \\
\hline & & & Unique & 71 & 48 & $1.68 \%$ \\
\hline & & \multirow{2}{*}{ Spleen Foxp $3^{+}$} & Total & 144 & 114 & \\
\hline & & & Unique & 82 & 49 & $3.08 \%$ \\
\hline & & \multirow{2}{*}{ CNS Foxp3- } & Total & 207 & 211 & \\
\hline & & & Unique & 82 & 45 & $1.65 \%$ \\
\hline & & \multirow{2}{*}{ CNS Foxp $3^{+}$} & Total & 155 & 207 & \\
\hline & & & Unique & 60 & 41 & $1.68 \%$ \\
\hline
\end{tabular}


$95 \%$ certainty of positive detection. For example, there were a total of 155 amino sequences in splenocyte Treg of pre-immune sample in experiment 1, if the frequency of particular CDR3 sequence in the population is above $2.25 \%$, the chance for it to be detected is $95 \%$. This indicates that the collected sequences were adequate to survey common sequences in each cellular cohort.

Among the V $\beta 8.2-\mathrm{J} \beta 2.7$ sequences obtained, 452 unique amino acid sequences were identified. Interestingly, $54(\sim 12 \%)$ of sequences were shared, or public sequences, among the 4 independent experiments. Public sequences were over-represented among more commonly identified sequences, with $74 \%$ of public sequences present within the 100 most frequent sequences. An increased probability of identifying sequences in multiple independent samples when their sequence frequency is high is expected, and implies that the observed proportion of public sequences underestimates total representation due to non-identification of low frequency public sequences. 16/54 public sequences were found in TCR exclusively used by Treg, 14/54 in Tconv TCR, and 24/54 in sequences identified both in Treg and Tconv. The CDR3 amino acid sequences of public sequences were diverse and no sequence patterns were identified.

Abundance coverage estimator analysis (ACE) of pooled sequences, an indicator of total sequence diversity, indicated a limited diversity consistent with findings in other systems, with values of 271 and 337 for pre-immune, and 191 and 171 for post-immune V $\beta 8.2^{+}, \mathrm{J} \beta 2.7^{+}$Tconv and Treg splenocytes respectively. This restricted diversity and the high proportion of public sequences indicates that the retrogenic repertoire is heavily sculpted and that few of the potential sequences that may form from V-D-J rearrangement are realized in the retrogenic mice. Interestingly, the original $\beta$ chain partner for the 1MOG244.2 $\alpha$ TCR (ASGDAGTGYEQY) was among the public sequences identified in the CNS Foxp3- populations of both EAE induction experiments, though not in pre-immune analyses. This suggests that receptors relevant to EAE in C57BL/6 mice, which served as the source of the 1MOG244.2 hybridoma, play a role in the 1 MOG $244.2 \alpha$ retrogenic mice.

Among public TCR, 20 CDR3 sequences were identified as common to both preimmune T-cells and T-cells from mice with EAE. TCR sequences appearing predominantly in Foxp $3^{+}$or Foxp $3^{-}$populations preimmune showed the same orientation, Foxp $^{+}$or Foxp3-, in mice with EAE for 19 of the 20 receptors. This implies that TCR sequence rather than disease status is the primary force governing T-cell Foxp3 expression.

\subsubsection{Limited Overlap of Treg and Tconv Repertoires}

Comparison of unique CDR3 amino acid sequences for each cellular cohort revealed several notable features. Repertoire overlap was greater among a single class of cells (Treg or Tconv) across different organs (spleen and CNS) than among different classes of cells within an organ (Figure 4-2A). In experiment 3, 32.7\% of unique splenic and CNS Treg sequences and $23.9 \%$ of unique splenic and CNS Tconv sequences 


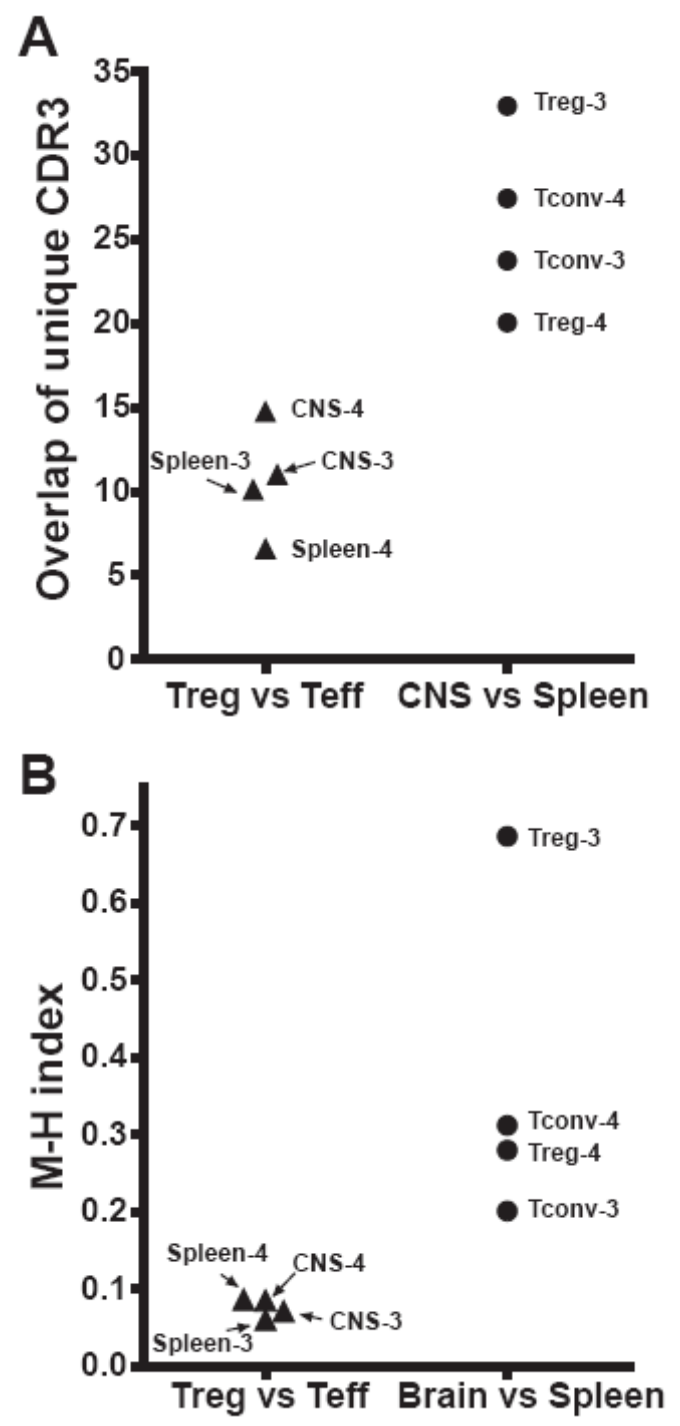

Figure 4-2. Population differences among TCR subsets in mice with EAE. (A) Unique TCR sequences from the identified experiments (Table 4-1) were compared between population groups, and the frequency of sequences shared by Treg and Tconv cells within an organ (left) or of sequences from a specific cell type, Treg and Tconv cells, shared across organs (right) plotted. (B) The Morisita-Horn index, a measure of ecologic diversity among populations, is plotted for the same groups as (A). A value of 1.0 indicates population identity. Whereas (A) plots sharing of uniquely identified sequences, (B) measures differences in both sequence diversity and identification frequency between the indicated populations. 
overlapped. In contrast, only $11.0 \%$ of unique CNS and $10.1 \%$ of unique splenic were shared between Treg and Tconv cells. In experiment 4 , the values were $20.0 \%, 27.4 \%$ $14.7 \%$, and $6.6 \%$, respectively. In addition, we used the Morisita Horn index to estimate similarity between populations. The Morisita Horn index is a commonly used, unitless quantitative index of the similarity between two populations (a value of 1.0 represents complete identity) that uses information regarding the number of species as well as the abundance of each species. The Morisita Horn index was widely used in T-cell repertoire studies before (Hsieh et al., 2006; Pacholczyk et al., 2002). Here, the M-H index also demonstrated strong dissimilarity in Treg and Tconv cell CDR3 sequences in either spleen or CNS and greater similarity when an individual cell type was compared between spleen and CNS (Figure 4-2B). Taken together, Treg and Tconv TCR repertoires are distinct, though do show some overlap.

To determine whether disease has an impact on the degree of overlap, we also compared the repertoires of preimmune and EAE mice. In preimmune experiment 1 and 2, the percent of unique shared TCRs between splenic Treg and Tconv cells was 3.5\% and $6.4 \%$ respectively. In EAE experiment 3 and 4 , the overlap was $10.1 \%$ and $6.6 \%$ in spleen, and $11.0 \%$ and $14.7 \%$ in CNS. Therefore there is a trend toward increasing overlap with disease development. However, this did not achieve statistical significance, $(p=0.28$ for preimmune versus EAE spleen, and $p=0.22$ for EAE spleen versus EAE CNS). This implies that autoimmune disease has limited impact on the overlap of Treg and Tconv TCR repertoire, and argues against substantial levels of intercoversion during autoimmunity.

Further, among the minority of unique sequences that were shared by Treg and Tconv cells, the isolates of a particular sequence were typically skewed toward a single class of cells, either Treg or Tconv cells. Indeed, the Treg/Tconv cell identification ratio of shared TCR should form a Gaussian distribution, potentially around a ratio of 1, if there was rapid, free and equal interconversion between Treg and Tconv cells. Yet $73 \%$ of shared CDR3 isolates were skewed by a ratio $\geq 2: 1$ toward a single cell class (Figure 4-3A), mean and median for majority Foxp3 ${ }^{+}$TCR: $6.1 \pm 7.3$, 2.5; Foxp3 ${ }^{-}$TCR: $9.1 \pm$ $11.4,5.1)$. This was influenced by the presence of CDR3 for which few sequences were isolated, and for which substantial skewing would therefore not be possible. Indeed, for shared sequences independently identified at least 10 times, $84 \%$ had a ratio $\geq 2: 1$ (Figure 4-3B; mean, median for Foxp3 ${ }^{+}: 9.3 \pm 8.4,9.1$; Foxp3 ${ }^{-}: 13.4 \pm 13.8,10.0$ ). Skewing of CDR3 toward a single cell class, Treg or Tconv cells, was also readily apparent in graphical mapping of the distribution among Foxp $3^{+}$or Foxp $3^{-}$cells of the most common sequences isolated in individual experiments (Figure 4-4 and 4-5).

Comparative analyses were further performed on three structural features of the identified CDR3, length, charge, and hydrophilicity as potential correlates of specificity. The six cohorts of unique CDR3 sequences, acquired from postimmune CNS, postimmune spleen, and preimmune spleen Foxp $3^{+}$and Foxp $3^{-}$cells were compared via the Kruskal-Wallis test and significance was observed only for charge $(p<0.0001)$. With the Wilcoxon rank-sum test to compare any two cohorts within this group, the charge of the CNS Foxp3 $3^{-}$CDR3 showed significant differences with each of the five other 

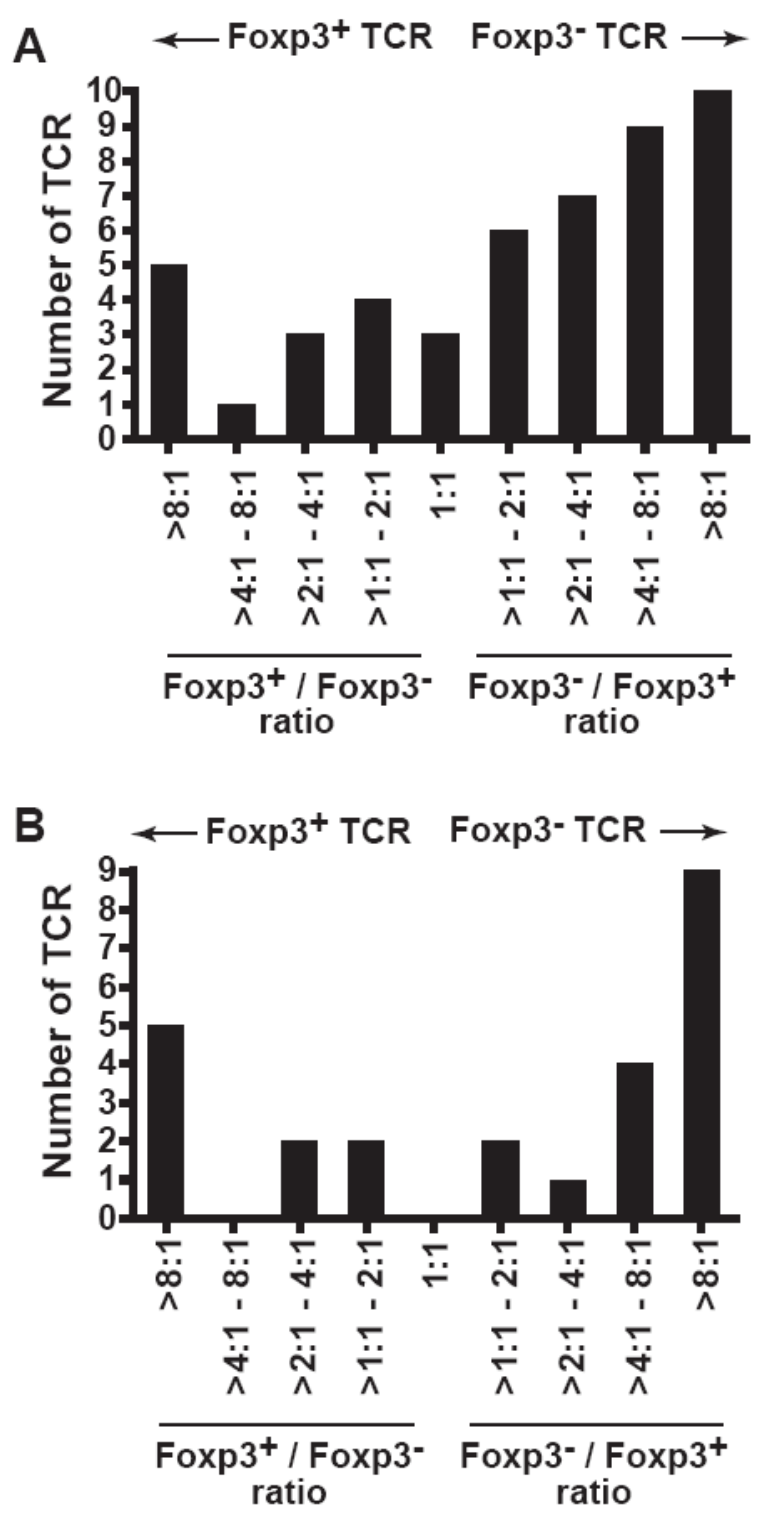

Figure 4-3. Distribution of shared CDR3 sequences. Numbers of CDR3 amino acid sequences identified in both Foxp $3^{+}$and Foxp $3^{-}$populations at the indicated ratio is shown. Left sides of plots indicate sequences that were primarily identified among Foxp $3^{+}$cells, and right sides indicate sequences primarily identified among Foxp $3^{-}$cells. A 1:1 ratio indicates equal numbers of sequences were identified among Foxp $3^{+}$and Foxp3 populations. (A) Data is plotted for all shared sequences identified. (B) Data is plotted only for those shared sequences identified 10 or more times. 
Figure 4-4. Frequency representation of the most common splenic $\mathrm{V} \beta 8.2^{+} \mathrm{J} \beta 2.7^{+}$

CDR3 isolates. Frequencies of the 10 most commonly isolated splenic CDR3 sequences from GFP-Foxp3 ${ }^{+}$(A) or GFP-Foxp3 ${ }^{-}$(B) T-cells in each of the 4 experiments is plotted (filled bars). CDR3 frequencies are shown as percent values and were defined as 100 * (Foxp $3^{+}$or Foxp $3^{-}$CDR3 isolates with the indicated sequence) / (total number of Foxp $3^{+}$ or Foxp3 ${ }^{-}$splenic CDR3 sequences for the indicated experiment). Experiments 1 and 2 assayed preimmune mice, and 3 and 4 studied mice with EAE. For sequences shared among Foxp $3^{+}$and Foxp $3^{-}$populations, below each filled bar the representation of the identical sequence in the alternative population is plotted, Foxp3 $3^{-}$cells in (A) and Foxp3 $3^{+}$ cells in (B) (unfilled bars). 
A $\mathrm{Foxp3}^{+} \mathrm{TCR}$

Pre-immune Foxp $3^{+}$, Expt 1
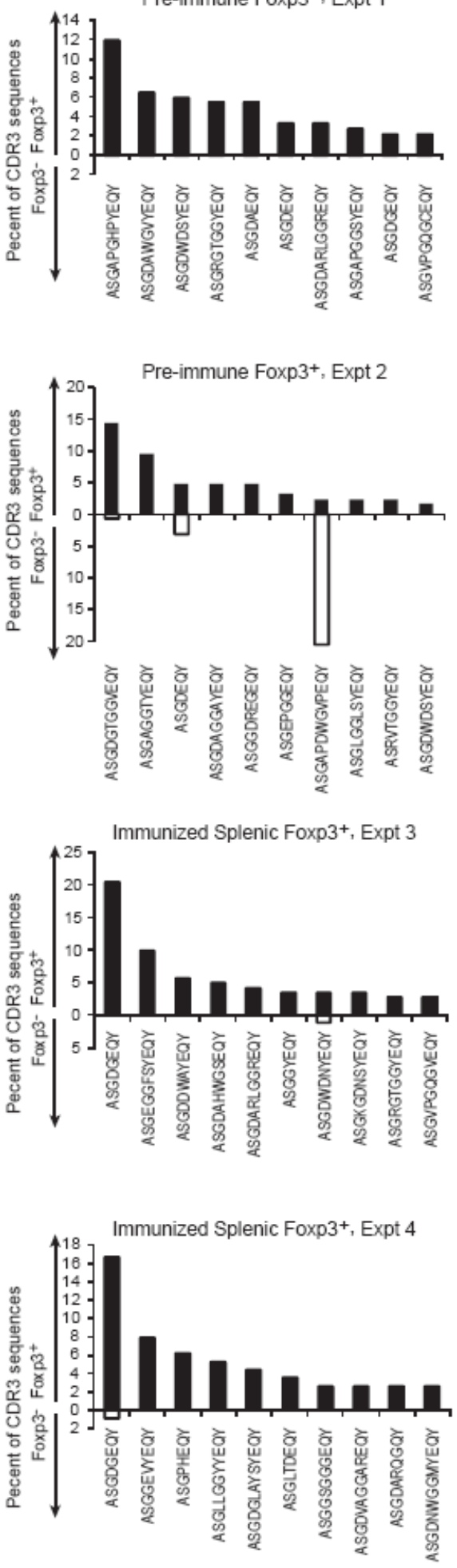

B Foxp3- TCR

Pre-immune Foxp3-, Expt 1
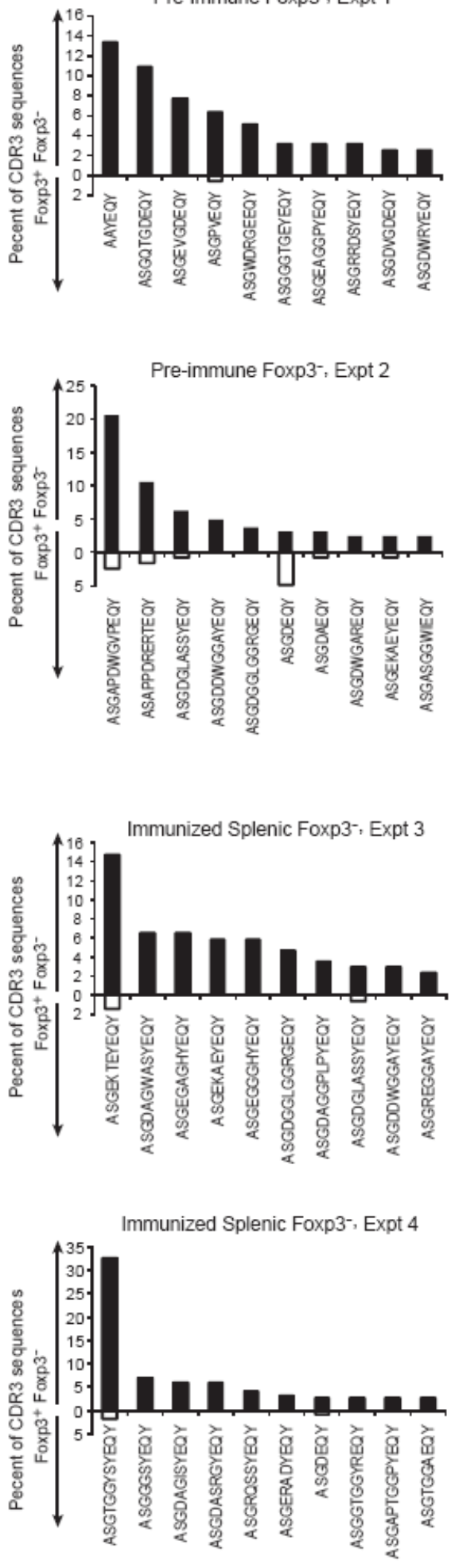

49 

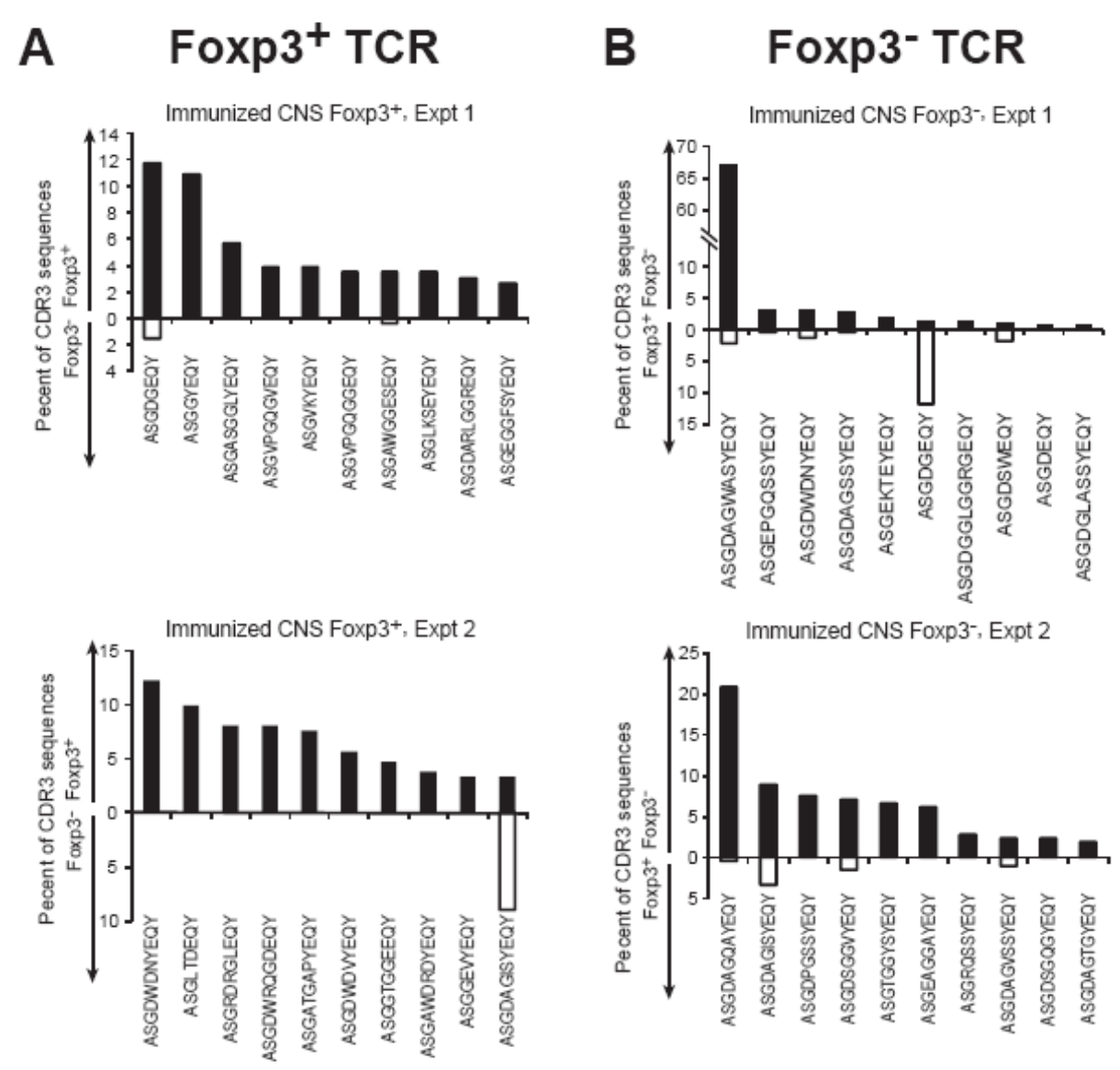

Figure 4-5. Frequency representation of the most common CNS-derived $\mathbf{V} \boldsymbol{\beta 8 . 2 ^ { + }} \mathbf{J} \beta 2.7^{+}$CDR3 isolates. Analyses were performed as in Figure 4-4, but for CNSderived sequences from experiments 3 and 4 . 
cohorts (Figure 4-6) ( $\mathrm{p} \leq 0.02$ for all comparisons after Hochberg's adjustment for multiple tests). A significant difference was seen in only one other comparison group, CNS and preimmune splenic Foxp $3^{+}$CDR3 $(p=0.019)$. Therefore, CNS-infiltrating Tcells, particularly Foxp $3^{-}$cells, possess at least one distinguishing CDR3 characteristic, increased negative charge. Of note, the $\mathrm{MOG}_{35-55}$ epitope bears a potentially complementary positive charge and includes three arginine residues, two of which are predicted to lie in the Ag binding groove with their guanidinium moieties directed toward the TCR (Ben-Nun et al., 2006).

\subsection{Summary}

We analyzed the Treg and Tconv cell TCR repertoires in EAE utilizing a retrogenic mouse model in which the TCR $\alpha$ chain locus is fixed. The overlap between Treg and Tconv cells was less than the overlap when a single class of cells was compared in two different organs - the spleen and CNS. This demonstrates that Treg and Tconv cells express largely distinct TCRs in the CNS and spleen of mice with EAE. Nevertheless, some overlap $(\sim 10 \%)$ was seen. This result echoed previous studies performed on unimmunized mice, which saw overlap between Treg and Tconv TCR repertoires, ranging from $10 \%$ to $20 \%$ (M-H index of 0.1-0.2) (Hsieh et al., 2004; Hsieh et al., 2006; Lathrop et al., 2008; Pacholczyk et al., 2006).

Comparison between preimmune and EAE mice revealed that the degree of overlap was not significantly increased in EAE. Among CDR $3 \beta$ shared by Treg and Tconv cells, individual sequences tended to be dominantly represented within a single cell type. These results argue against large scale interconversion in an inflammatory environment. It is consistent with previous results, in which little conversion was found after the adoptive transfer of $\mathrm{CD}^{+} \mathrm{CD} 25^{-} \mathrm{T}$-cells (Lathrop et al., 2008) and in TCR repertoire analysis of NOD mice (Wong et al., 2007a). In contrast it has also been suggested that conversion contributes to $\sim 72 \%$ of peripheral Treg cells (Liang et al., 2005).

Public TCRs were identified in the V $\beta 8.2-\mathrm{J} \beta 2.7$ repertoire shared in the four independent experiments (preimmune and EAE mice). This is consistent with the previous report that T-cells express $\mathrm{V} \alpha$ and $\mathrm{V} \beta$ public repertoires in the CNS of C57BL/6 mice during MOG-EAE (Fazilleau et al., 2006). Further the original 1MOG244.2TCR was among the public sequences identified in the CNS Foxp3 $3^{-}$group, implying that this particular TCR is important to EAE in retrogenic and C57BL/6 mice.

In summary, these results and the identification of distinct charge characteristics specifically among CNS-infiltrating Tconv cells implies that Treg and Tconv cells in EAE are distinct populations and do not substantially interconvert. 

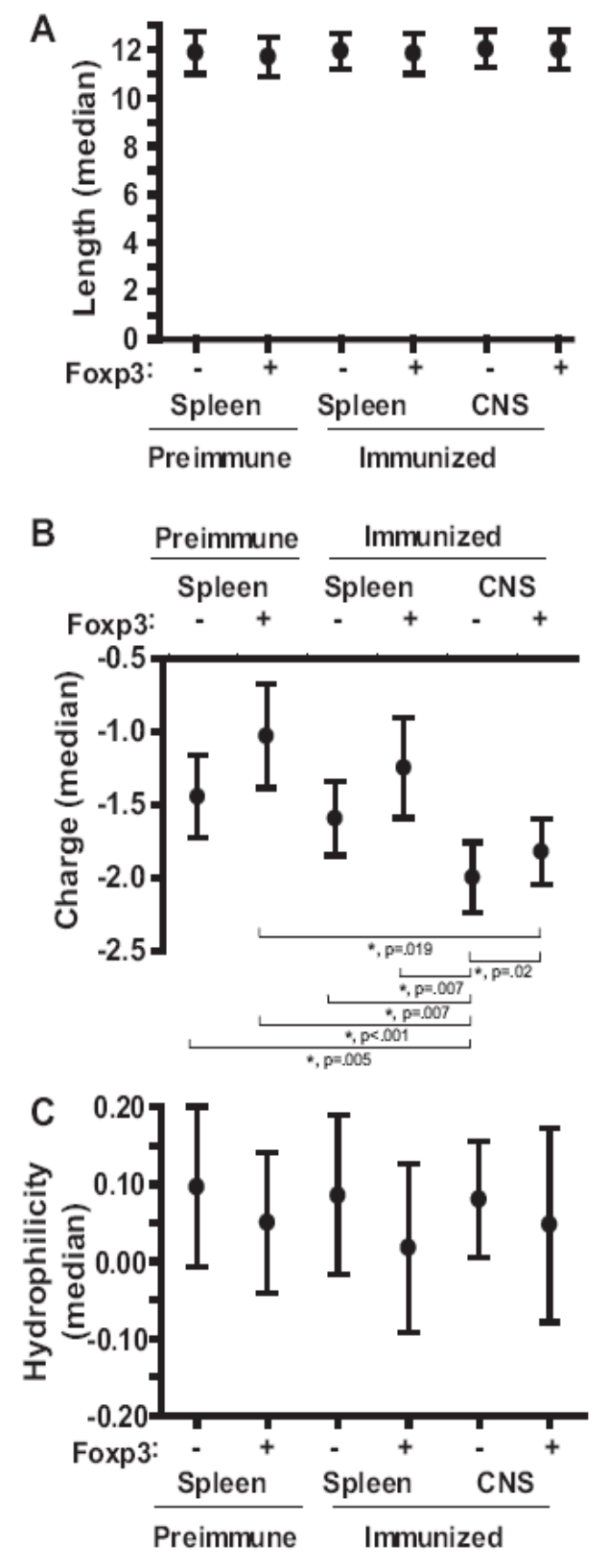

Figure 4-6. Length, charge, and hydrophilicity of isolated CDR3. Medians $\pm 95 \% \mathrm{CI}$ for length (A), charge (B), and hydrophilicity (C) among unique CDR3 sequences identified in the six indicated populations is plotted. Analyzed sequences were determined as the regions bounded by but not including the conserved $\mathrm{C}$ and $\mathrm{F}$ residues bordering each CDR3 $\beta$. ${ }^{*} \mathrm{p}<0.05$ according to the Hochberg-adjusted Wilcoxon rank sum test. 


\section{CHAPTER 5. ANALYSIS OF TCR IN 1MOG244.2TCR $\alpha$ RETROGENIC MICE}

\subsection{Introduction}

T-cell development begins in the thymus, and one of the earliest events is TCR gene rearrangment. The mature thymocyte repertoire is formed based on the ability of the TCRs to react with host MHC molecules, which are mostly occupied with selfpeptides (Starr et al., 2003). Continued interactions between TCRs and peptide-MHC molecules contribute to the maintenance and homeostatic expansion of mature T-cells in the periphery (Bender et al., 1999; Ernst et al., 1999), thus reshaping the peripheral T-cell repertoire. These processes allow the immune system to maintain a T-cell repertoire containing sufficient diversity and specificity to respond against an unknown and highly diverse pathogen pool.

In Chapter 4 we showed that Treg repertoire is highly diverse with distinct set of TCRs compared with Tconv cells, though with some overlap between populations both in unimmunized mice and in mice with EAE. However, the role of TCR specificity in directing thymic precursors into the Treg or Tconv linage remains unclear. Some studies indicated TCR specificity is crucial to Treg differentiation. T-cells exclusively expressing the transgenic TCR appeared incapable of undergoing $\mathrm{CD} 4{ }^{+} \mathrm{CD} 25^{+}$Treg cells selection. However, the introduction of cognate peptide into the repertoire of selfpeptides can induce the formation of $\mathrm{CD} 4{ }^{+} \mathrm{CD} 25^{+}$Treg cells expressing the clonotypic transgenic TCR. For example, Treg cell development was seen when TS1 TCR transgenic mice were mated with mice containing cognate ligands (HA28). The formation of Treg cells has also been described in mice expressing HA under the control of an immunoglobulin promoter in RAG-deficient backgrounds (Apostolou et al., 2002; Jordan et al., 2001). Likewise, when DO11.10 TCR transgenic mice were crossed with $\mathrm{RAG}^{-/-}$mice expressing cognate peptide - ovalbumin (OVA), $\mathrm{CD} 4{ }^{+} \mathrm{CD} 25^{+}$Treg cells were also formed (Kawahata et al., 2002; Walker et al., 2003). These findings suggested that the activation by agonist ligands in thymus could promote Treg cell formation. This together with the utilization of different TCRs in Treg than Tconv cells implies that thymocytes possessing self-antigen specificity are more likely to develop into Treg cells. On the other hand, Treg cells can recognize a wide range of foreign antigens, have a diverse TCR repertoire, and no increased reactivity of Treg cells toward self was identified in one analysis of T-cell hybridomas derived from Treg and Tconv cells (Pacholczyk et al., 2007). Therefore, we cannot completely exclude the possibility that TCRs with weak affinity to self or that are not self-reactive will be included in Treg repertoire.

Though TCR repertoire analysis is powerful in picturing the overall relationship between Treg and Tconv cells, it is limited in that inferences are made by population shifts in the absence of specific knowledge about antigen specificity. Likewise, studies of antigen reactivity among Treg and Tconv cells have typically been performed outside of the context of repertoire analyses that illuminate relationships between T-cell populations. To better clarify the specificity and responsiveness of Treg cells, in this 
chapter, we focused the studies on a cohort of TCRs identified in Chapter 4, reconstituted into the $\mathrm{CD}^{+}{ }^{\mathrm{TCR}} \alpha \beta$-deficient 4G4.CD4 T hybridoma. We were thereby able to determine the frequency of MOG-specific auto-reactive TCRs in Treg and Tconv cells populating the CNS and spleen. The experiments answered the following questions: Are Treg or Tconv cells present in the CNS during MOG-EAE more prone to be MOGspecific than those in the spleen, and the frequencies of MOG-specific cells differ between Treg and Tconv cells. What are the sensitivity and fine specificity differences among the self-reactive Treg and Tconv TCRs? Are there differences in the CDR3 features of MOG-specific TCR from Treg and Tconv cells? This analysis may give clues as to how the nature of the TCR itself impacts on the fate of Tcells: to become Treg or Tconv? Localize to CNS or periphery?

\subsection{Results}

\subsubsection{MOG-specificity among Treg and Tconv TCR}

To better delineate the properties of the responding TCR present within the CNS and periphery during EAE, we recreated full-length $\alpha \beta$ TCR cDNAs from 40 identified CDR3 sequences (Table 5-1). The CDR3 were selected from sequences identified at high frequency (all were among the 50 most commonly identified sequences) to provide an approximately equal distribution of predominantly Foxp $3^{+}$and Foxp $3^{-}$CDR3 of splenic or CNS origin. As above (Figures 4-3, 4-4 and 4-5), TCR with sequences shared by both Treg and Tconv cells were heavily skewed toward a single cell class (Table 5-1, far right column). A high representation of public TCR was seen among the selected CDR3 (21/40 sequences), reflecting the increased frequency of these sequences among common TCR. Reconstituted TCR were subcloned into a tricistronic retroviral vector essentially as we have previously described (Alli et al., 2008), with TCR $\alpha$ and $\beta$ chains linked by the Thosea asigna virus $2 \mathrm{~A}$ sequence to facilitate their stoichiometric expression and an IRES-linked cyan fluorescent protein (CFP). TCR-encoding retrovirus for these and the original 1MOG244.2 $\alpha \beta$ TCR were transduced into the CD4 ${ }^{+}$TCR $\alpha \beta$-deficient 4G4.CD4 T-cell line (Figure 5-1). The cells were flow cytometrically sorted for similar TCR expression levels and stimulated with $\mathrm{MOG}_{35-55}$ peptide, and IL-2 production was measured as an indicator of MOG responsiveness.

MOG-specificity varied among the TCR cohorts (Figure 5-2). 2/12 (16.7\%) of splenic Foxp3 ${ }^{-}$TCR from immunized mice responded to MOG, whereas 1/8 (12.5\%) of splenic Foxp ${ }^{+}$TCR responded. In contrast to this more modest representation of MOGspecific cells in the periphery, 8/8 (100\%) of CNS Foxp3 $3^{-}$and 6/12 (50\%) of CNS Foxp $3^{+}$TCR-transduced cells produced IL-2 in response to MOG. These results therefore demonstrate substantial enrichment of MOG-specificity within CNS-infiltrating Tconv cells of mice with EAE. Further, despite the evidence from our repertoire analyses for limited interconversion between Tconv and Treg cells and the use of a TCR $\alpha$ in the retrogenics derived from a Foxp $3^{-}$T-cell, a substantial proportion of the Treg cell 
Table 5-1. Features of CDR3 $\beta$ used to reconstitute full-length TCR cDNA.

\begin{tabular}{|c|c|c|c|c|c|c|c|c|c|}
\hline $\begin{array}{l}\text { Clone } \\
\#\end{array}$ & Site & $\begin{array}{l}\text { MOG- } \\
\text { specific }\end{array}$ & Foxp $3^{+}$ & Sequence & Length & Public & $\begin{array}{l}\text { Foxp3 } 3^{+} \\
\text {TCR }\end{array}$ & $\begin{array}{l}\text { Foxp3- } \\
\text { TCR }\end{array}$ & $\begin{array}{l}\text { Foxp3 } 3^{+} / \text {Foxp3 } \\
\text { ratio }\end{array}$ \\
\hline 4 & Spl & $\mathrm{N}$ & $\mathrm{N}$ & ASGDGGLGGRGEQY & 14 & Y & 0 & 18 & \\
\hline 7 & Spl & $\mathrm{N}$ & $\mathrm{N}$ & ASGEKAEYEQY & 11 & $\mathrm{Y}$ & 1 & 15 & 0.067 \\
\hline 10 & Spl & $\mathrm{N}$ & $\mathrm{N}$ & ASGTGGYSYEQY & 12 & $\mathrm{~N}$ & 2 & 82 & 0.024 \\
\hline 17 & Spl & $\mathrm{N}$ & $\mathrm{N}$ & ASGRQSSYEQY & 11 & $\mathrm{~N}$ & 0 & 15 & \\
\hline 30 & Spl & $\mathrm{N}$ & $\mathrm{N}$ & ASGGGSYEQY & 10 & $\mathrm{~N}$ & 0 & 17 & \\
\hline 31 & Spl & $\mathrm{N}$ & $\mathrm{N}$ & ASGAPDWGVPEQY & 13 & $\mathrm{~N}$ & 3 & 33 & 0.090 \\
\hline 32 & Spl & $\mathrm{N}$ & $\mathrm{N}$ & ASGEKTEYEQY & 11 & $\mathrm{~N}$ & 2 & 30 & 0.067 \\
\hline 36 & Spl & $\mathrm{N}$ & $\mathrm{N}$ & ASGDDWGGAYEQY & 13 & $\mathrm{Y}$ & 6 & 14 & 0.43 \\
\hline 37 & Spl & $\mathrm{N}$ & $\mathrm{N}$ & ASGDGLASSYEQY & 13 & $\mathrm{Y}$ & 2 & 20 & 0.1 \\
\hline 47 & Spl & $\mathrm{N}$ & $\mathrm{N}$ & ASGPVEQY & 8 & $\mathrm{Y}$ & 1 & 13 & 0.077 \\
\hline 5 & $\mathrm{CNS}$ & $\mathrm{N}$ & $\mathrm{Y}$ & ASGDWDNYEQY & 11 & $\mathrm{Y}$ & 35 & 10 & 3.5 \\
\hline 8 & $\mathrm{CNS}$ & $\mathrm{N}$ & $\mathrm{Y}$ & ASGGYEQY & 8 & $\mathrm{Y}$ & 33 & 2 & 16.5 \\
\hline 15 & $\mathrm{CNS}$ & $\mathrm{N}$ & $\mathrm{Y}$ & ASGRDRGLEQY & 11 & $\mathrm{~N}$ & 18 & 0 & \\
\hline 20 & $\mathrm{CNS}$ & $\mathrm{N}$ & $\mathrm{Y}$ & ASGDWDVYEQY & 11 & $\mathrm{~N}$ & 14 & 0 & \\
\hline 34 & $\mathrm{CNS}$ & $\mathrm{N}$ & $\mathrm{Y}$ & ASGDGTGGVEQY & 12 & $\mathrm{Y}$ & 26 & 1 & 26 \\
\hline 39 & CNS & $\mathrm{N}$ & $\mathrm{Y}$ & ASGDARLGGREQY & 13 & $\mathrm{Y}$ & 20 & 0 & \\
\hline 3 & Spl & $\mathrm{N}$ & $\mathrm{Y}$ & ASGDGEQY & 8 & $\mathrm{Y}$ & 86 & 8 & 10.8 \\
\hline 14 & Spl & $\mathrm{N}$ & $\mathrm{Y}$ & ASGGEVYEQY & 10 & $\mathrm{~N}$ & 16 & 0 & \\
\hline 24 & Spl & $\mathrm{N}$ & $\mathrm{Y}$ & ASAGWGLYEQY & 11 & $\mathrm{~N}$ & 7 & 0 & \\
\hline 26 & Spl & $\mathrm{N}$ & $\mathrm{Y}$ & ASGEGGFSYEQY & 12 & $\mathrm{~N}$ & 20 & 0 & \\
\hline 28 & Spl & $\mathrm{N}$ & $\mathrm{Y}$ & ASGDDWAYEQY & 11 & $\mathrm{~N}$ & 13 & 0 & \\
\hline 41 & $\mathrm{Spl}$ & $\mathrm{N}$ & $\mathrm{Y}$ & ASGRGTGGYEQY & 12 & $\mathrm{Y}$ & 19 & 0 & \\
\hline 46 & Spl & $\mathrm{N}$ & $\mathrm{Y}$ & ASGDAWGVYEQY & 12 & $\mathrm{Y}$ & 15 & 0 & \\
\hline
\end{tabular}


Table 5-1. Continued.

\begin{tabular}{|c|c|c|c|c|c|c|c|c|c|}
\hline $\begin{array}{l}\text { Clone } \\
\#\end{array}$ & Site & $\begin{array}{l}\text { MOG- } \\
\text { specific }\end{array}$ & Foxp $^{+}$ & Sequence & Length & Public & $\begin{array}{l}\text { Foxp3 } 3^{+} \\
\text {TCR }\end{array}$ & $\begin{array}{l}\text { Foxp3 } \\
\text { TCR }\end{array}$ & $\begin{array}{l}\text { Foxp3 } 3^{+} / \text {Foxp3 }^{-} \\
\text {ratio }\end{array}$ \\
\hline 6 & CNS & $\mathrm{Y}$ & $\mathrm{N}$ & ASGEPGQSSYEQY & 13 & $\mathrm{Y}$ & 2 & 12 & 0.17 \\
\hline 11 & CNS & $\mathrm{Y}$ & $\mathrm{N}$ & ASGDAGISYEQY & 12 & $\mathrm{Y}$ & 7 & 32 & 0.22 \\
\hline 12 & CNS & $\mathrm{Y}$ & $\mathrm{N}$ & ASGDAGSSYEQY & 12 & $\mathrm{Y}$ & 1 & 8 & 0.13 \\
\hline 19 & CNS & $\mathrm{Y}$ & $\mathrm{N}$ & ASGDSGGVYEQY & 12 & $\mathrm{Y}$ & 3 & 15 & 0.2 \\
\hline 21 & CNS & $\mathrm{Y}$ & $\mathrm{N}$ & ASGDAGQAYEQY & 12 & $\mathrm{Y}$ & 1 & 45 & 0.022 \\
\hline 29 & CNS & $\mathrm{Y}$ & $\mathrm{N}$ & ASGEAGGAYEQY & 12 & $\mathrm{Y}$ & 3 & 14 & 0.21 \\
\hline 45 & CNS & $\mathrm{Y}$ & $\mathrm{N}$ & ASGDPGSSYEQY & 12 & $\mathrm{~N}$ & 0 & 16 & \\
\hline 44 & Spl & $\mathrm{Y}$ & $\mathrm{N}$ & ASGDASRGYEQY & 12 & $\mathrm{~N}$ & 0 & 16 & \\
\hline 50 & Spl & $\mathrm{Y}$ & $\mathrm{N}$ & ASGEGGGHYEQY & 12 & $\mathrm{Y}$ & 0 & 12 & \\
\hline $244.2 *$ & Spl & $\mathrm{Y}$ & $\mathrm{N}$ & ASGDAGTGYEQY & 12 & $\mathrm{Y}$ & 0 & 7 & \\
\hline 1 & CNS & $\mathrm{Y}$ & $\mathrm{Y}$ & ASGASGGLYEQY & 12 & $\mathrm{~N}$ & 13 & 1 & 13 \\
\hline 9 & CNS & $\mathrm{Y}$ & $\mathrm{Y}$ & ASGLTDEQY & 9 & $\mathrm{~N}$ & 25 & 0 & \\
\hline 16 & CNS & $\mathrm{Y}$ & $\mathrm{Y}$ & ASGATGAPYEQY & 12 & $\mathrm{~N}$ & 16 & 0 & \\
\hline 18 & CNS & $\mathrm{Y}$ & $\mathrm{Y}$ & ASGVPGQGVEQY & 12 & $\mathrm{~N}$ & 13 & 0 & \\
\hline 52 & CNS & $\mathrm{Y}$ & $\mathrm{Y}$ & ASGAPGGSYEQY & 12 & $\mathrm{Y}$ & 11 & 0 & \\
\hline 110 & CNS & $\mathrm{Y}$ & $\mathrm{Y}$ & ASGVPGQGGEQY & 12 & $\mathrm{~N}$ & 11 & 0 & \\
\hline 35 & Spl & $\mathrm{Y}$ & Y & ASGAPGHPYEQY & 12 & $\mathrm{Y}$ & 22 & 0 & \\
\hline
\end{tabular}

Note: Clone \#, site of primary isolation, MOG-specificity, association with Foxp3 expression, CDR3 length, isolation from multiple experiments (public) and frequency of identification from Foxp $3^{+}$and Foxp3 ${ }^{-}$cDNA is indicated. *, original 1MOG244.2TCR. 

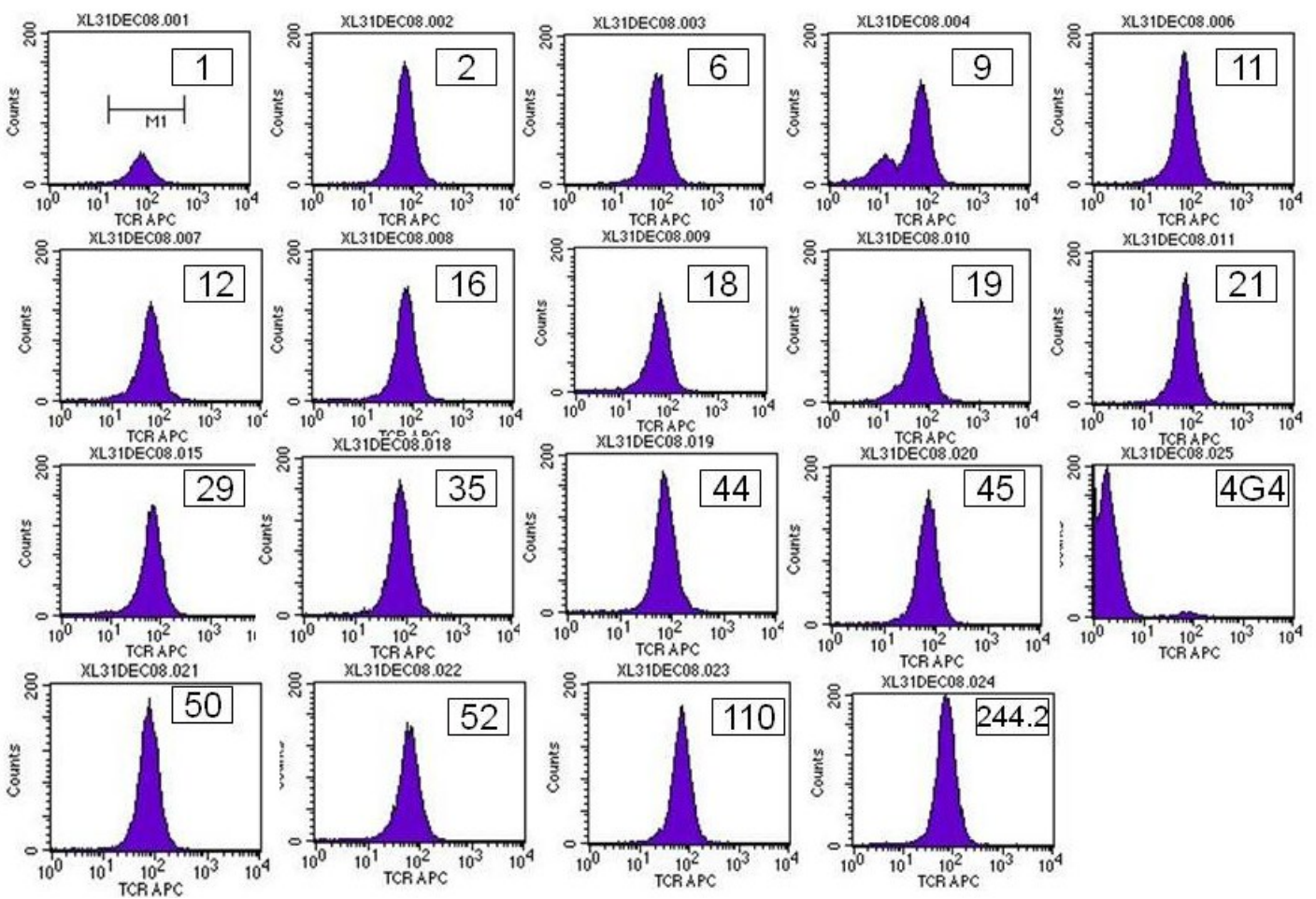

Figure 5-1. Expression of TCR on CD4.4G4 cells. TCR was retrovirally transduced into CD4.4G4 cells and cytometrically sorted for $\mathrm{CD}^{+} \mathrm{TCR}^{+}$population. Sorted cells were expanded for 3-4 days, and TCR expression was analyzed using anti-TCR $\beta$ antibody. 

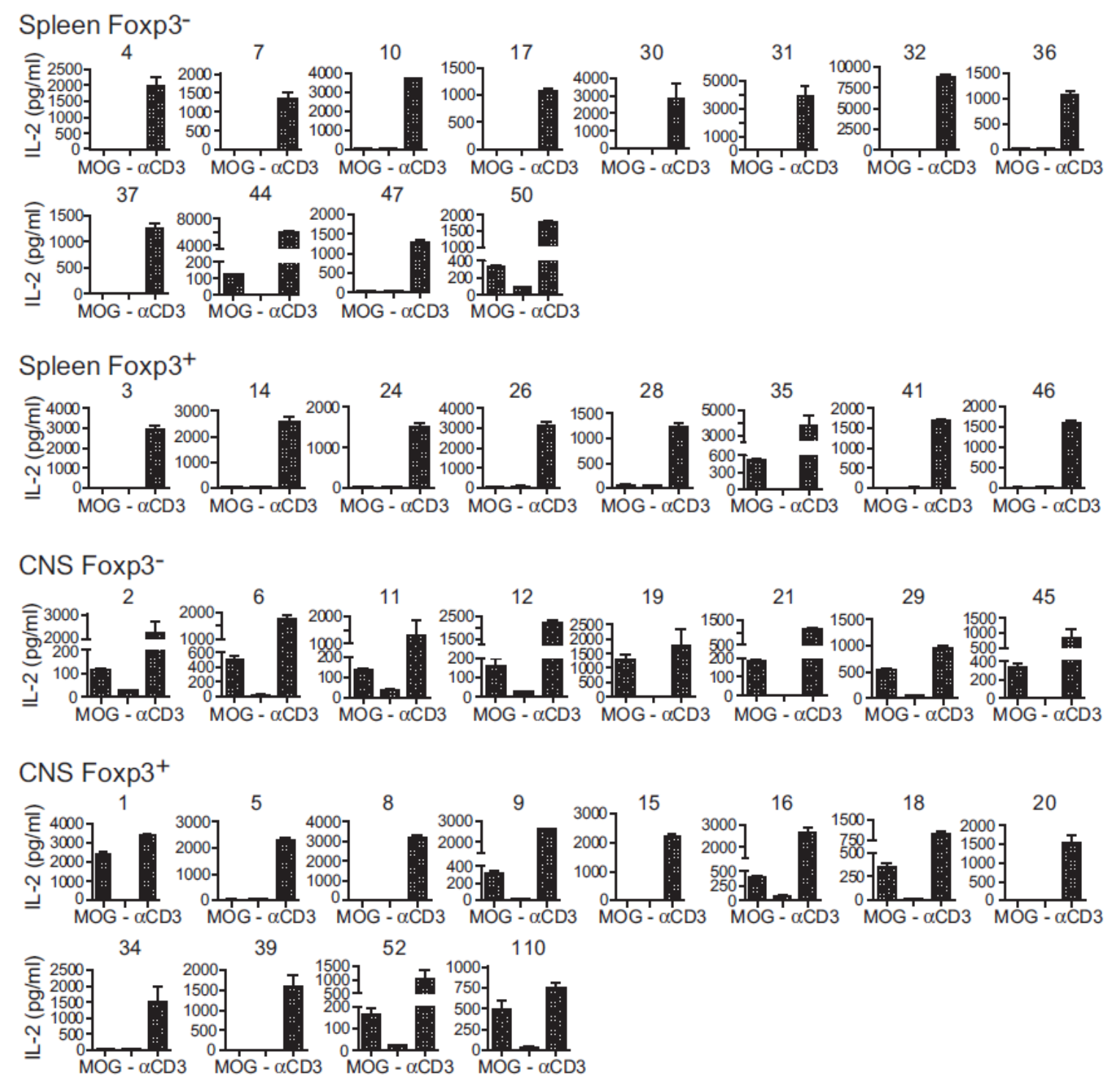

Figure 5-2. MOG-specificity among isolated TCR. Forty CDR3 $\beta$, distributed among Foxp $^{+}$and Foxp $3^{-}$isolates from the CNS or spleen, were used to construct retrovirus incorporating full-length $\alpha \beta$ TCR. Retrovirally transduced 4G4.CD4 T-cells were flow cytometrically sorted for similar surface expression of TCR and stimulated with $\mathrm{Tcra}^{-/-}$ splenic APC with or without $100 \mu \mathrm{g} / \mathrm{ml} \mathrm{MOG}_{35-55}$ peptide or with plate-bound CD3 antibody. IL-2 production was measured in cell-free supernatant at $24 \mathrm{hr}$ by ELISA. Data indicate mean +1 SEM and are representative of three or more assays for each TCR. 
response was directed against MOG. This indicates that regulatory T-cells in MOG-EAE respond to cognate autoantigen.

\subsubsection{Segregation of MOG-Specific Treg and Tconv Cell TCR Based on CDR3ß Characteristic}

Sequence information for all MOG-specific and nonspecific TCR was compared (Table 5-1). This provided several insights. First, MOG-specific CDR3 $\beta$, Foxp $3^{+}$or Foxp3 ${ }^{-}$, bore a consistent length of $12(11.9 \pm 0.8 ; 15 / 18$ [83\%] sequences length of 12$)$. The non-MOG-specific TCR showed only slightly decreased average length $(11.2 \pm 1.6)$, but many fewer 12 amino acid sequences $(5 / 23 ; 23 \%)$ and more diversity in this measure. MOG-specific TCR further more consistently had a $G$ at the $6^{\text {th }}$ CDR3 position (16/18; $89 \%$ ) compared with non-MOG-specific TCR (4/23; 17\%), implying that the absence of a side chain in that position is important for antigen recognition. More interestingly, among the MOG-specific TCR, CDR3 sequence distinguished TCR derived from primarily Foxp3 $3^{+}$and Foxp3 $3^{-}$T-cells. Acidic D or E residues were present at position 4 in $11 / 11(100 \%)$ of Foxp3 $3^{-}$but $0 / 7(0 \%)$ of Foxp $3^{+}$MOG-specific CDR3. D and E would be anticipated to have a high prevalence at this location; a D is formed with V $\beta 8.2-\mathrm{D} \beta$ recombination in the absence of junctional mutations or with a $\mathrm{C} \rightarrow \mathrm{T}$ replacement at the first D-region nucleotide. An $\mathrm{E}$ is formed with a $\mathrm{C} \rightarrow \mathrm{A}$ or $\mathrm{G}$ replacement at the same nucleotide. Indeed, 5/10 (50\%) of Foxp $3^{-}$and 8/13 (62\%) of Foxp $3^{+}$nonspecific CDR3 bore a $\mathrm{D}$ or $\mathrm{E}$ at position 4 , making its complete absence more surprising in the Foxp $3^{+}$ MOG-specific population. In contrast, $100 \%$ of the MOG-specific Foxp ${ }^{+}$TCR bore an aliphatic A (4/7), V (2/7), or L (1/7) residue at that location, residues seen in only 1 of the 34 other CDR3 examined. Therefore, TCR expressed by MOG-specific Treg and Tconv cells comprise distinct families. Implicitly, these cells undergo a distinct selection process in 1MOG244.2 $\alpha$ mice that is either not based on MOG recognition or is based on differential recognition of MOG.

\subsubsection{Sensitivity and Fine Specificity of MOG-specific TCR for Auto-antigen}

Current models of Treg cell lineage specification suggest that Treg cells possess a proclivity for self-reactivity and that thymocytes with higher affinity for self-antigen are diverted into the Foxp ${ }^{+}$lineage (Pacholczyk and Kern, 2008). MOG is expressed in the thymus, though in small amounts (Delarasse et al., 2003). If MOG is responsible for the selection of MOG-reactive Treg cells, then these cells would be anticipated to bear an increased affinity for it. Because the 4G4.CD4 TCR transductants were essentially identical except for the sequence of their TCR CDR3 $\beta$, their relative sensitivity to Ag should primarily reflect their affinity. We therefore tested the sensitivity of all MOGspecific TCR to dilutions of $\mathrm{MOG}_{35-55}$ peptide (Figure 5-3). Interestingly, although Treg cell TCR trended toward increased sensitivity, substantial overlap was observed between the MOG-specific Treg and Tconv cell TCR. In each case, receptors were identified with high, moderate, and low sensitivity for MOG. Moreover, specific pairs of Foxp $3^{+}$and Foxp3 ${ }^{-}$CDR3 with conserved amino acid sequences showed similar sensitivities for 

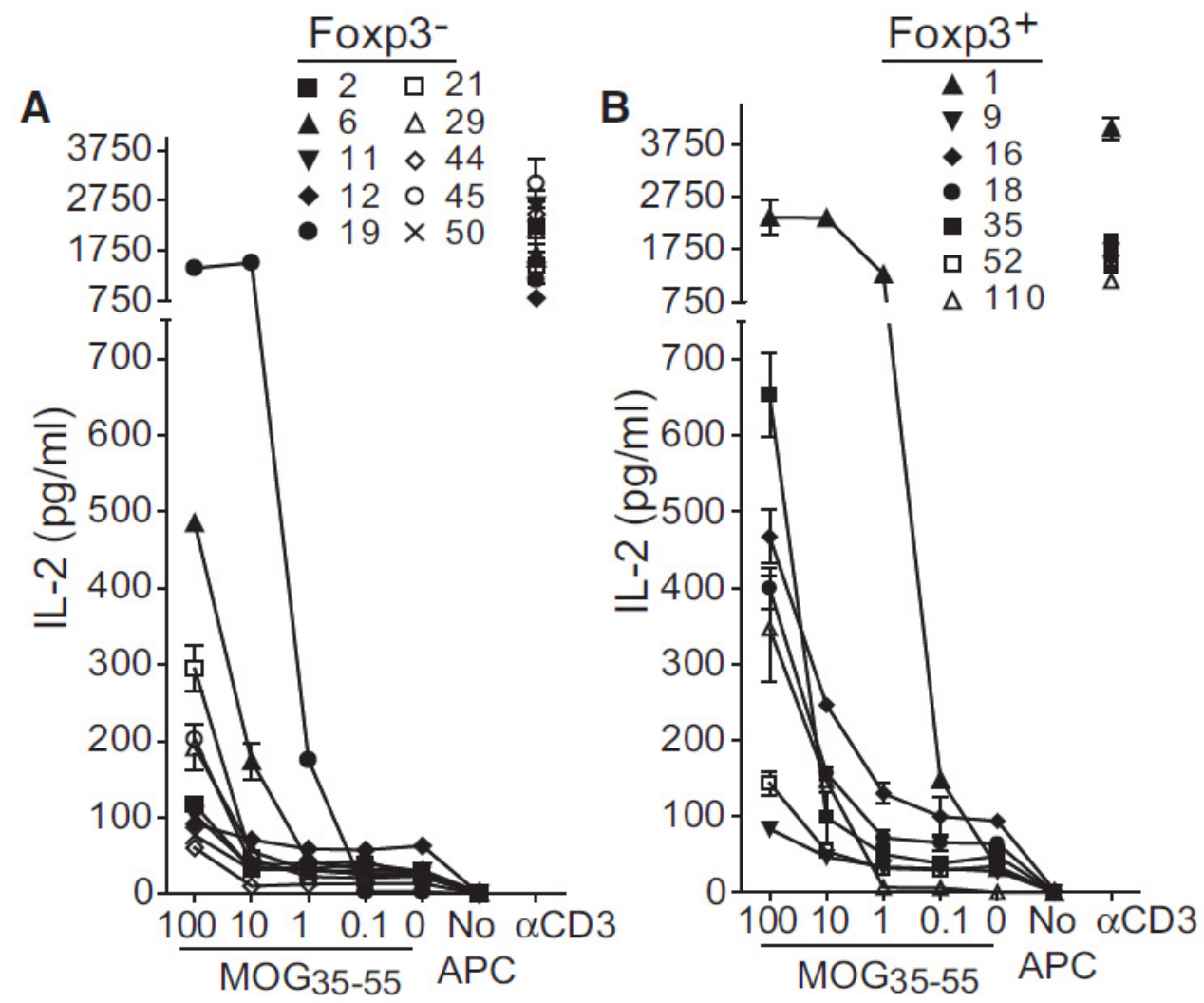

Figure 5-3. Sensitivity of MOG $_{35-55}$-specific TCR for autoantigen. MOG-specific 4G4.CD4 transductants were assayed for relative sensitivity to antigen. Cells bearing Foxp3 $^{-}$(A) or Foxp3 ${ }^{+}$(B) T-cell derived TCR were analyzed as in Figure 5-1 but stimulated with varied doses of $\mathrm{MOG}_{35-55}$ peptide. All cell lines were simultaneously analyzed for comparability. Data shown is representative of 3 independent experiments with at least 2 independent transductions of each TCR. 
antigen (Table 5-1 and Figure 5-3). Clones 19 (Foxp3 ${ }^{-}$) and 1 (Foxp3 ${ }^{+}$), which besides the variation at position 4 only have a conserved $\mathrm{V} \rightarrow \mathrm{L}$ substitution at position 8 , both responded vigorously to MOG stimulation. Clones 45 (Foxp3 ${ }^{-}$) and 52 (Foxp3 ${ }^{+}$), which differ only at position 4 and by a $\mathrm{S} \rightarrow \mathrm{G}$ at position 7 , also responded similarly and more moderately. Therefore, although Treg cells tend toward increased sensitivity for MOG, this does not seem to be encoded into the position 4 variation and a MOG sensitivity threshold does not appear fundamental to response by Treg versus Tconv cell TCR.

These results suggest that other factors affected by the position 4 variation, for instance differential fine specificity for MOG or sensitivity to an alternative crossreactive selecting $\operatorname{Ag}(\mathrm{s})$, are responsible for Treg cell lineage assignment. To test the former possibility, we examined the fine specificity of recognition of five of the MOGspecific Foxp3 ${ }^{-}$TCR, including the index 1MOG244.2TCR, and four of the Foxp3 ${ }^{+}$TCR. Prior studies with $\mathrm{MOG}_{35-55}$-specific TCR have identified critical TCR interacting residues, particularly R41, F44, R46, and V47 (Petersen et al., 2004). The different TCR demonstrated variability, through common patterns of reactivity when tested with alanine-substituted $\mathrm{MOG}_{35-55}$ peptides (Figure 5-4). An R41A substitution did not substantially influence recognition for $1 \mathrm{MOG} 244.2$ or most of the other TCR, whereas response was diminished to F44A, R46A, and V47A substitutions. However, with these and alanine substitutions at all residues from V37 to L50, no pattern was identified that distinguished the fine specificity of the Foxp3 ${ }^{+}$and Foxp3 ${ }^{-}$T-cell-derived TCR. Therefore, differential recognition of cognate MOG antigen does not appear to be associated with the binary differentiation of T-cells with acidic or aliphatic residues at CDR3 $\beta$ position 4 alternatively into Tconv or Treg cells. Considering the absence of evidence for affinity or fine specificity-based segregation of Tconv and Treg cell TCR, our data would suggest that a MOG sensitivity threshold is not itself responsible for MOG-specific Treg cell recruitment, and presumably also lineage assignment.

\subsubsection{Fidelity in TCR Lineage Specification}

The 1MOG244.2 $\alpha$ retrogenic mice were produced with $\mathrm{Tcra}^{-/-} \mathrm{HPC}$ donor and recipient mice. We were interested whether the MOG-specific Treg and Tconv cell TCR that we identified in 1MOG244.2 $\alpha$ mice (Table 5-1) would similarly guide differentiation in a distinct system where the thymus is from a wild-type and not $\mathrm{Tcra}^{-/-}$mouse and most developing T-cells are unmanipulated. To test this, we transduced MOG-specific TCR $\alpha \beta$ pairs into CD45.1 ${ }^{-} \mathrm{CD} 45.2^{+} \mathrm{Rag}^{-/}$HPC, which cannot express other TCR. These were diluted with an excess of congenic CD $45.1^{+} \mathrm{CD} 45.2^{-}$T-cell-depleted bone marrow cells and transplanted into $\mathrm{CD} 45.1^{+} \mathrm{CD} 45.2^{-}$mice. Seventeen mice demonstrated detectable $\mathrm{CD} 45.2^{+} \mathrm{CD}^{+} \mathrm{T}$-cell engraftment (range $0.001 \%-8.5 \%$ of $\mathrm{CD}^{+} \mathrm{TCR}^{+}$cells), including mice expressing three TCR derived from Treg cells (\#1, 9, 18, Table 5-1) and four from Tconv cells $(\# 2,6,21,45)$ (Figure 5-5). Overall, the Treg cell-derived TCR showed $>20$ fold increased engraftment into the Foxp $3^{+}$lineage when compared with the Tconv cell TCR (mean of Foxp3 ${ }^{+}$cells: $21.4 \% \pm 10.6 \%$; of Foxp3 ${ }^{-}$cells: $0.95 \% \pm 1.1 \%$ ). Therefore, cells expressing TCR derived from Tconv cell TCR in 1MOG244.2 $\alpha$ mice differentiate into Tconv and not Treg cells in this system. Cells expressing TCR derived from Treg 

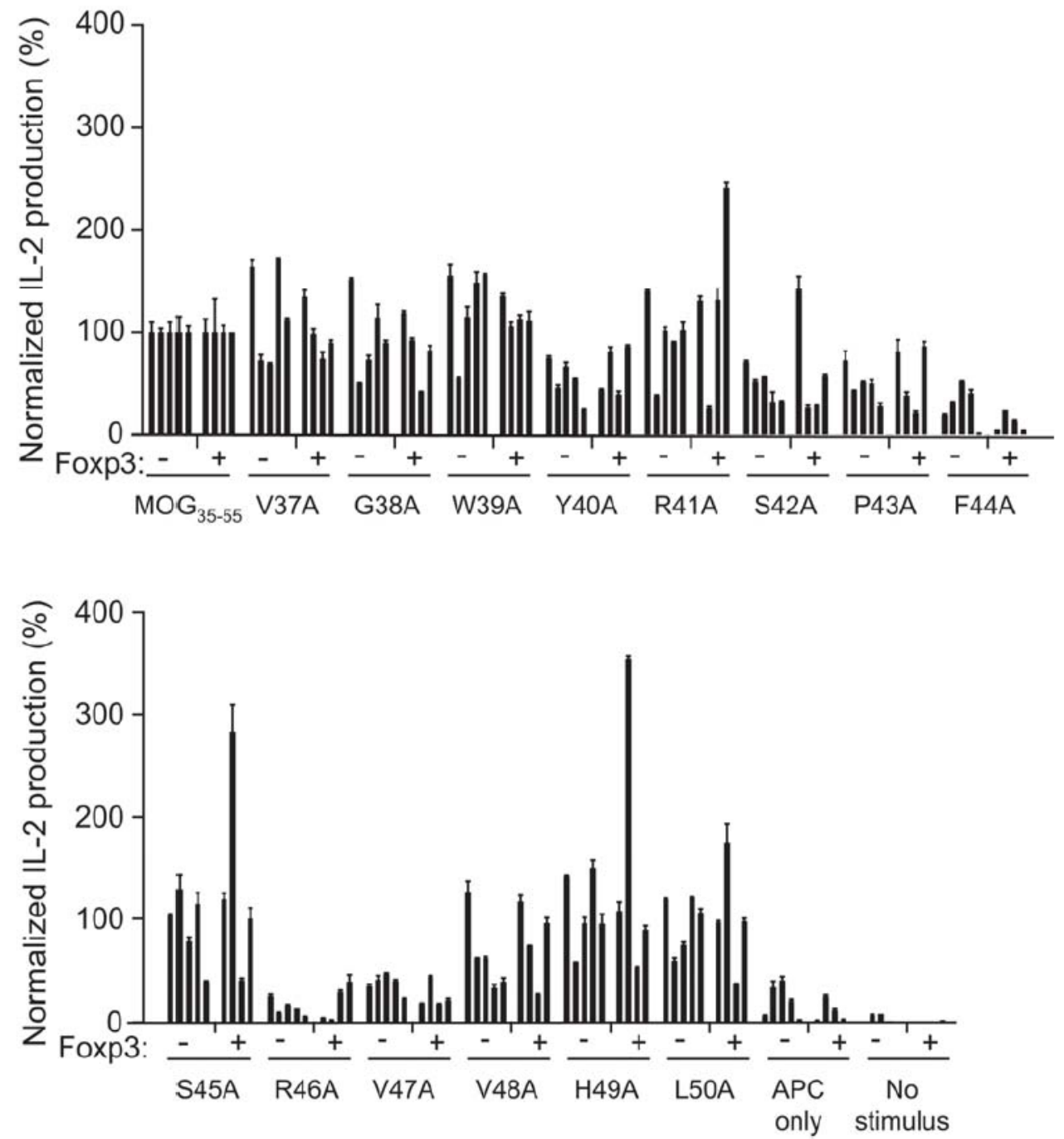

Figure 5-4. Fine specificity of Foxp3 ${ }^{+}$and Foxp3- TCR. The indicated 4G4.CD4 cell lines expressing Foxp $3^{+}$or Foxp3 $3^{-}$-derived TCRs were stimulated with irradiated Tcro ${ }^{-/-}$ APCs in the absence of antigen or in the presence of $100 \mu \mathrm{g} / \mathrm{ml} \mathrm{MOG}_{35-55}$ or the indicated alanine-substituted mutants of this peptide. IL-2 was measured in cell free supernatant at $24 \mathrm{~h}$ as an indicator of stimulation. Percent IL-2 production compared with $\mathrm{MOG}_{35-55}$ stimulation is plotted. Bars indicate from left to right IL-2 production by clones $6,21,29,45,244.2,1,16,18$ and 35 after stimulation with the indicated peptide. Mean + SEM is plotted. 
A
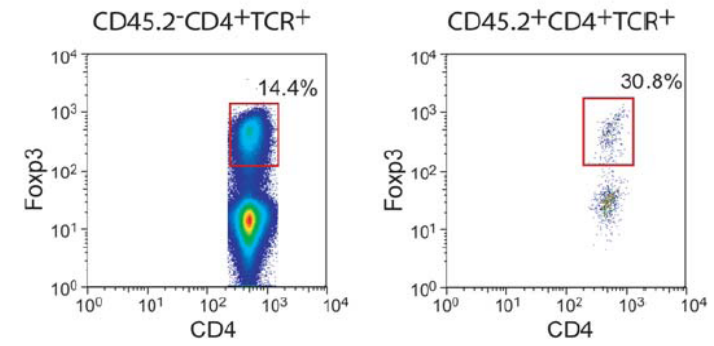

B

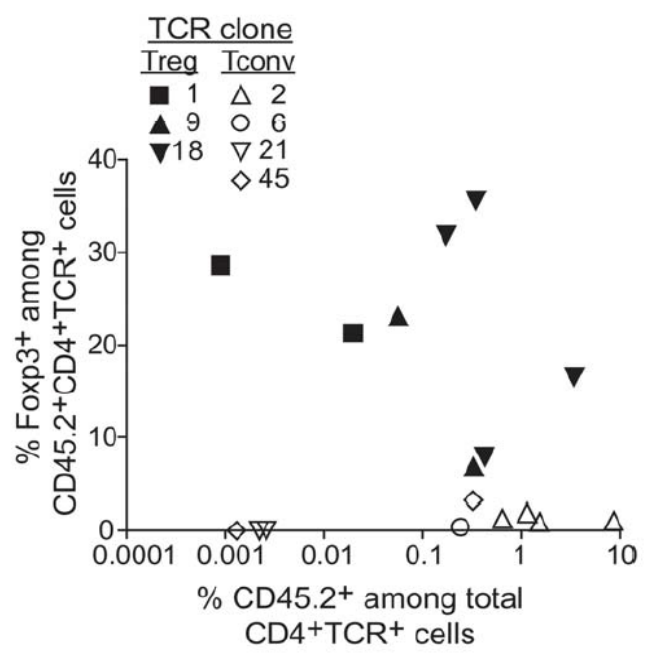

Figure 5-5. Foxp3 expression among CD4 ${ }^{+} \mathrm{T}$-cells in TCR-retrogenic mixed chimeras. To evaluate the lineage preference of MOG-specific Treg or Tconv TCR identified from1MOG244.2 $\alpha$ mice in mice with a wild-type thymus and few transgenic precursor cells, we produced mixed chimeras using select cloned TCR. Retroviral constructs incorporating the TCR $\alpha \beta$ from MOG-specific Treg-derived TCR clones 1, 9, and 18, and Tconv derived clones2, 6, 21, and 45 were transduced into B6.129S7$\operatorname{Rag} 1^{\text {tm1Mom }} / \mathrm{J}$ HPC. The Rag1 deficiency ensures that all T-cells developing from the transduced population express the TCR transgene. An $\sim 1-5 \%$ transduction efficiency was obtained. The HPC were mixed at a 1:1 or 1:10 ratio with untransduced, wild-type, T-cell depleted CD45.1 congenic B6.SJL-Ptprc ${ }^{\mathrm{a}}$ Pep3 $3^{\mathrm{b}}$.BoyJ-derived bone marrow, and transplanted into B6.SJL-Ptprc ${ }^{\mathrm{a}}$ Pep $3^{\mathrm{b}}$.BoyJ mice. At 6-8 weeks after transplant, mice were sacrificed, and splenocytes stained with CD45.2, TCR, CD4, and intracytoplasmic Foxp3 antibodies. (A) Flow cytometric analysis shows Foxp3 expression on CD45.2 ${ }^{-} \mathrm{CD}^{+} \mathrm{TCR}^{+}$or $\mathrm{CD} 45.2^{+} \mathrm{CD}^{+} \mathrm{TCR}^{+}$gated populations. (B) Engraftment and percent Foxp3 positivity among TCR-transduced CD $45.2^{+}$T-cells is indicated. Percent engraftment of $\mathrm{CD} 4{ }^{+} \mathrm{CD} 45.2^{+} \mathrm{T}$-cells was calculated as $100 \mathrm{x}$ (number of $\mathrm{CD} 45.2^{+} \mathrm{CD}^{+} \mathrm{TCR}^{+}$cells identified) / (total number of $\mathrm{CD}^{+} \mathrm{TCR}^{+}$cells). Percent $\mathrm{Foxp} 3^{+}$ T-cells is calculated as $100 \times$ (number of CD45.2 $2^{+} \mathrm{CD}^{+} \mathrm{TCR}^{+} \mathrm{Foxp} 3^{+}$cells) / (total number of CD $45.2^{+} \mathrm{CD}^{+} \mathrm{TCR}^{+}$cells). 
cell TCR in 1MOG244.2 $\alpha$ mice show increased Treg cell bias. Although not all of the retrogenic T-cells expressing the Treg cell-derived TCR developed into Foxp $3^{+}$cells, this is not surprising because niche saturation for Treg cell development may occur even at very small numbers of Ag-specific T-cells (Bautista et al., 2009). Despite heavy dilution with wild-type cells in these mixed chimeric mice, cells expressing the assayed TCR should nevertheless be increased in frequency compared with 1MOG244.2 $\alpha$ mice. Therefore, Treg and Tconv cell lineage assignment in the 1MOG244.2TCR $\alpha$ retrogenic mice is paralleled in a distinct and arguably more natural system, one in which the thymic stroma and the large majority of T-cells are wild-type.

\subsection{Summary}

In this chapter, we selected 40 high frequency TCRs identified in Chapter 4, transduced them into CD4.4G4 hybridomas, and analyzed their responsiveness to $\mathrm{MOG}_{35-55}$. We found that autoreative Tconv cells were highly enriched in the CNS ( $100 \%$ of TCR), but not spleen, of 1 MOG244.2TCR $\alpha$ mice with EAE. $50 \%$ of CNSinfiltrating Treg cells were also MOG-reactive. This result confirms the presence of auto-antigen reactive T-cells accumulating in CNS of mice with EAE, and indicates that a large percentage of Treg TCR are MOG-reactive.

MOG-specific and non-specific TCRs showed distinct CDR3 $\beta$ sequence features. MOG-specific TCRs uniformly bear a length of 12 amino acids, and a glycine at position 6. More importantly, among MOG-specific TCRs, Treg and Tconv TCR possess distinct characteristics. Aspartic acid or glutamic acid at position 4 were exclusively seen in Tconv TCR, whereas aliphatic residues - alanine, valine and leucine were found in Treg TCR. This suggests that Treg and Tconv cells during EAE arise from distinct population repertoire.

Analysis of sensitivity differences between MOG-specific Treg and Tconv cells showed similar and overlapping responsiveness. Further differential fine specificity for $\mathrm{MOG}_{35-55}$ was not identified in Treg and Tconv TCRs. This implies two possibilities; one is that $\mathrm{MOG}_{35-55}$ is not responsible for T-cells lineage assignment and alternative $\mathrm{Ag}(\mathrm{s})$ should be considered, the other is that affinity threshold is not fundamental for Treg cell selection.

To ensure the lineage commitment of Treg and Tconv cells seen in 1MOG244.2TCR $\alpha$ mice can also be observed in a more normal thymic environment, we transplanted highly diluted $\mathrm{Rag}^{-/-}$HPCs expressing MOG-specific Treg or Tconv TCRs into congenic wild-type mice. The same lineage specification was readily observed as in retrogenic mice, suggesting that TCR-driven Treg and Tconv cell differentiation identified in 1MOG244.2TCR $\alpha$ mice is indicative of the differentiation potentials of the same TCR in normal thymus as well. 


\section{CHAPTER 6. DISCUSSION}

\subsection{Introduction}

In Chapters 3-5, we demonstrated through studies of repertoire and individual TCR only limited overlap between Treg and Tconv cells responding during MOG-EAE. MOG-specific Treg cells formed a substantial though not exclusive component of the CNS-infiltrating Treg cell repertoire. Examination of MOG-specific Treg and Teff cell TCR with a fixed TCR $\alpha$ and restricted TCR $\beta$ usage failed to find evidence for sensitivity or fine specificity for MOG influencing Treg versus Tconv cell lineage assignment, but did reveal lineage segregation based on conserved CDR3 features. Our results favor a model in which Treg cell response during EAE is developmentally encoded in the TCR repertoire and argue against anything more than limited plasticity between Treg and Tconv cells during the autoinflammatory response.

In this chapter we will discuss the following problems and future plans: Are 1MOG244.2TCR $\alpha$ mice an optimal model to study T-cell repertoire? Does interconversion play an important role in maintaining Treg population in the inflammatory environment? What is the source of CNS-infiltrating Treg cells in MOGEAE? Do self-reactive Treg cells show higher sensitivity to self-antigen than Teff cells do? Is MOG the only antigen CNS-infiltrating T-cells recognize in MOG-EAE? Does epitope spread and cross-reactivity influence the regulatory response in MOG-EAE?

\subsection{Developing a Retrogenic Model of EAE to Facilitate Repertoire Analysis}

\subsubsection{Why Does the TCR $\alpha$ Chain Need to be Fixed?}

The purpose of fixing one TCR chain is to limit TCR variability and help determine the specificity of TCR repertoire. With one TCR chain fixed, it is only necessary to define the second chain's sequence, eliminating the need to clone both chains from a single cell. Previous data showed that TRBV13-2 (V $\beta 8.2)$ was highly associated with auto-reactivity in EAE, with nearly half of auto-reactive MOG-specific T-cells expressing V $\beta 8.2$ (Mendel et al., 1995; Mendel Kerlero de Rosbo and Ben-Nun, 1996; Offner et al., 1992), whereas TRAV use (Vo) is much more variable. By fixing the TCR $\alpha$ chain and studying TCR $\beta$, we are able to focus on the disease relevant V $\beta 8.2^{+}$ TCR population. In addition, the CDR3 $\beta$ region shows greater diversity (V-N-D-N-J) than the CDR3 $\alpha(\mathrm{V}-\mathrm{N}-\mathrm{J})$, suggesting that the TCR $\beta$ chain will have increased variability compared with the TCR $\alpha$. A bias towards particular V $\beta$ gene families was also seen in virus infection, autoimmunity and alloreactivity models (Aebischer et al., 1990; Baker et

al., 2002; Callan et al., 1996; Kedzierska et al., 2004; Oksenberg et al., 1993; Pantaleo et al., 1994; Torres-Nagel et al., 1997; Trautmann et al., 2005). This might be a consequence of the limited number of available mouse TCR V $\beta$ gene segments (35) 
compared with a greater number of mouse V $\alpha$ gene segments (71) (Lefranc et al., 1999). Thus studying TCR $\beta$ chain repertoire will potentially yield valuable information, particularly on the MOG-specific response.

\subsubsection{Does a Fixed a Chain Influence the Course of MOG-EAE Significantly?}

One concern in retrogenically fixing TCR $\alpha$, is that the timing of TCR $\alpha$ chain expression in the thymus is not physiological and may affect the repertoire. In thymic development, TCR $\beta$ chain rearranges first in DN cells, followed by the formation of $\mathrm{TCR} \beta / \mathrm{pT} \alpha / \mathrm{CD} 3 / \mathrm{TCR} \zeta_{2}$ complexes. The pre-TCR triggers the signal transduction cascade by activating the kinase Lck, engaging the adaptor SLP-76 and linker of activated T-cells (LAT), and thus activating protein kinase C, Ras, and MAP kinases (Gartner et al., 1999; Hagenbeek et al., 2004; Iritani et al., 1999; Schmedt and Tarakhovsky, 2001). Comparison of T-cell development in mice with a TCR $\alpha$ transgene and lacking $\mathrm{pT} \alpha$, and mice lacking a transgene but with wild-type $\mathrm{pT} \alpha$ suggests that TCR $\alpha \beta$ complexes are less effective than pT $\alpha$ complexes in supporting proliferation, survival, and $\alpha \beta$ T-cell lineage commitment of early T-cell progenitors. This is due to intrinsic differences in signaling capability of the different complexes (Baldwin et al., 2005; Borowski et al., 2004; Huang and Kanagawa, 2004; Lacorazza et al., 2001). To avoid this circumstance, many repertoire analyses have been performed on TCR $\beta$ transgenic mice, studying the TCR $\alpha$ repertoire (Hsieh et al., 2004; Pacholczyk et al., 2006; Wong et al., 2007b)

For the reasons listed above, we nevertheless selected to fix the TCR $\alpha$ rather than TCR $\beta$ chain. To evaluate the impact of the retroviral transgene on T-cell development, we conducted flow cytometeric analyses of thymocytes from 1MOG244.2 retrogenic and wild-type C57BL/6 mice. No significant difference of DN, DP, SP subpopulations between retrogenic and WT mice were seen between retrogenic and WT mice. More significantly the DN3 to DN4 transition, when pT $\alpha$ signaling appears most important did not appear affected by the 1MOG244.2 $\alpha$ transgene (Figure 3-2). This suggests that the early TCR $\alpha$ expression does not inhibit thymocytes from passing through the DN3 to DN4 checkpoint, or diminish the efficiency of positive selection in 1MOG244.2 retrogenic mice.

We did observe repertoire alterations in the periphery of retrogenic compared with wild-type mice. First, different use of V $\beta$ segments in 1MOG244.2TCR $\alpha$ retrogenic mice was observed. For example, V $\beta 14$ was substantially increased in retrogenic mice ( $\mathrm{p}$ $<0.05$ ), and V $\beta 3, V \beta 4, V \beta 8.3$ were decreased (Figure 3-4). Second, the peripheral Tcell repertoire in $1 \mathrm{MOG} 244.2 \mathrm{TCR} \alpha$ is skewed to the CD4 compartment. The ratio of CD4/CD8 T-cells reaches 5-10 in LN and spleen, implying that the transduced $\alpha$ chain favors $\mathrm{CD}^{+} \mathrm{T}$-cell selection or expansion in the periphery. A similar skewing to the CD4 lineage has been seen in C6 TCR $\alpha$ Tg female mice (Furmanski et al.). Third, retrogenic mice with different $\alpha$ transgenes have distinct susceptibilities to EAE (Figure 3-1A). Four different retrogenic mice expressing distinct $\alpha$ chains were challenged with 
MOG, and only 1MOG244.2TCR $\alpha$ showed EAE that caused peak disease levels comparable to WT mice, though they have delayed onset of disease. These results suggest that the $\alpha$ transgene has potential impact on T-cell selection and may alter the repertoire to some extent.

Does this change influence our experimental results? It is to be expected that the manipulation inherent in fixing TCR $\alpha$ will limit the repertoire of TCR $\beta$, as many rearranged TCR $\beta$ will not pair with this TCR $\alpha$. V $\beta$ distribution in splenic Tconv cells is as diverse as WT mice, and V $\beta 8.1 / 8.2$ is predominately used. After immunization with $\mathrm{MOG}_{35-55}$ peptide, $1 \mathrm{MOG} 244.2 \alpha$ develops EAE with similar disease severity as WT, and CNS-infiltrating T-cells predominately use V $\beta 8.1 / 8.2$ as described in C57BL/6 mice (Mendel et al., 1995; Mendel Kerlero de Rosbo and Ben-Nun, 1996). Most importantly, the percent of Treg among $\mathrm{CD}^{+}{ }^{\mathrm{T}}$-cells in thymus, LNs, and spleen is $3.20 \% \pm 1.06 \%$, $12.84 \% \pm 2.24 \%$, and $7.50 \% \pm 2.41 \%$ respectively, which is similar to those in wild-type mice. This argues against impaired development of Treg cells. Taken together, the repertoire is altered in 1MOG244.2 $\alpha$ mice, but remains diverse. Further, that diversity is present in both the Treg and Tconv populations. Nevertheless, it is important to experimentally document that the Treg versus Tconv lineage specification we observed in 1MOG244.2TCR $\alpha$ mice would be similarly seen in a more normal situation (Section 5.2.4), and these studies are ongoing.

\subsubsection{Fixing the TCR $\alpha$}

T-cell receptor (TCR) transgenic mice are widely used and have had a large contribution to understanding of T-cell development and signal transduction. Previous repertoire analyses relied on transgenic mice which had fixed $\beta$ chain with or without a TCR $\alpha$ chain minilocus (Hsieh et al., 2004; Malherbe et al., 2004; Wong et al., 2007b). However, TCR transgenic mouse production is time consuming and costly.

To avoid this, we utilized retrogenic mice in which specific TCRs are retrovirally transduced into hematopoietic stem cells (Holst et al., 2006b). This approach is substantially faster than making trangegnic mice, ( 6 weeks versus 6 months), and engraftment is reproducible from mouse to mouse. Also, mice containing different fixed $\alpha$ chains can be examined simultaneously. Since these mice are individually generated and each of them is a founder, the potential bias introduced by a founder effect would be limited (Holst et al., 2006b). However, there are still several disadvantages to retrogenic mice. For instance, the number of T-lymphocytes obtained from retrogenic mice is generally 3-4 fold less than that from Tg mice. Pooling mice was necessary to obtain enough CNS-infiltrating Treg cells for RNA extraction and TCR repertoire analysis. Retrogenic mice have to be constantly generated. Also, because T-cells first develop in transplanted adult mice (6-8 weeks) rather than the neonatal environment, an increased memory-like phenotype of peripheral T-cells may result as T-cells form in a lymphopenic environment. Finally, we sub-lethally irradiated recipient mice to facilitate stem cell engraftment, and irradiation itself can generate inflammation and enhanced cytokine secretion that may last up to 6 weeks (Holst et al., 2006b). Although we have not seen 
any deleterious consequences per se, these effects can potentially influence experimental results and should be considered in interpreting data from the system.

\subsection{Interconversion and Origins of Treg and Tconv Cells in Autoimmunity}

The Treg cells largely originate in the thymus. Seemingly, encounter with selfantigens at intermediate affinity induces Treg cell differentiation (Apostolou et al., 2002; Hsieh et al., 2004; Jordan et al., 2001; van Santen et al., 2004). This results in the generation of a largely distinct TCR repertoire in Treg and Tconv cells, as indicated by studies of TCR CDR3 $\alpha$ sequences in transgenic systems. On the other hand, a noticeable overlap, ranging from 10 to $42 \%$ between Treg and Tconv is also observed (Hsieh et al., 2004; Hsieh et al., 2006; Pacholczyk et al., 2006; Pacholczyk and Kern, 2008; Wong et al., 2007b). One hypothesis is that interconversion may play a role in generating the overlap between Treg and Tconv cells, and this interconversion involves either upregualtion of Foxp3 in Tconv or loss of Foxp3 in Treg cells (Hoffmann et al., 2009).

The conversion of Tconv into Treg can be achieved both by TGF $\beta$ and IL-2 or retinoic acid (RA) exposure during activation in vitro (Chen et al., 2003; Wang et al., 2009), or chronic exposure to antigen delivered by osmotic pumps or by antibody mediated targeting in vivo (Apostolou and von Boehmer, 2004; Cobbold et al., 2004; Knoechel et al., 2005; Kretschmer et al., 2005). Mechanistic studies have provided a framework to explain the plasticity between Treg and Tconv cells, with selective signaling pathways regulating Foxp3 expression (Coombes et al., 2007; Mucida et al., 2007; Quintana et al., 2008; Selvaraj and Geiger, 2007). In addition, conversion was readily seen in the studies involving adoptive transfer of purified $\mathrm{CD} 4{ }^{+} \mathrm{CD} 25^{-}$or $\mathrm{CD}^{+}{ }^{+}$Foxp3 $3^{-}$T-cells into lymphopenic or lymphoreplete hosts, with conversion varying from $0.3 \%$ to $12 \%$ (Curotto de Lafaille et al., 2004; Fontenot et al., 2005b; Lathrop et al., 2008; Liang et al., 2005). If one extrapolates the behavior of the adoptively transferred population as representative of their respective T-cell population, $0.4 \%$ and $8 \%$ conversion in periphery of normal mice would contribute to $3.6 \%$ and $72 \%$ of the Treg cells, respectively (Lathrop et al., 2008). This is a very large range and the higher numbers are not consistent with other repertoire studies. Thus conversion in the periphery was seen in several studies, though to what extent it maintains the peripheral Tcell population is still controversial.

Whether interconversion influences active immune responses, and more specific cells participating in autoimmunity, is uncertain. Some results suggest that adaptive upregulation of Foxp3 plays an important role in modulating autoimmunity (Manicassamy et al., 2009; You et al., 2007). Other studies failed to identify a role for Foxp3 induction (Korn et al., 2007; Wong et al., 2007a). During EAE, T-cells activated in the periphery migrate to the CNS where they re-engage antigen on local dendritic cells and expand (Bailey et al., 2007). The early CNS response has a low frequency of Foxp3 ${ }^{+}$ T-cells, suggesting that substantial numbers of Treg cells are not stimulated to enter the $\mathrm{CNS}$ at this time. Yet Treg cells accumulate later in the disease and are associated with resolution (McGeachy et al., 2005). Therefore, either migration or expansion of this 
population is delayed or CNS-infiltrating Treg cells are forming through Tconv cell conversion.

There are several possible explanations for how interconversion can influence Tconv and Treg cells in MOG-EAE. First interconversion may be a rare event in lymphoid organs with little target antigen. But once challenged by MOG, the T-cells will migrate to the CNS where the activated T-cells will encounter antigen in varied settings, and interconversion may occur. With this possibility we would expect to observe little overlap of Tconv and Treg in preimmune and little to moderate overlap in postimmune spleen, and much greater overlap in CNS (Figure 6-1A). A second possibility is that interconversion occurs frequently in periphery of normal individuals, and inflammation may or may not change the interconversion rate. In this case we would observe substantial overlap between Tconv and Treg cells in preimmune spleen, postimmune spleen and CNS (Figure 6-1B). A third possibility is that interconversion occurs infrequently in unimmunized organs, and antigen exposure or inflammatory environment does not facilitate interconversion. In this case, we would observe a limited overlap between Treg and Tconv in preimmune spleen, postimmune spleen and CNS (Figure 6-1C). Finally, it is possible that interconversion occurs most prominently with the initial immunization. In this case, we would expect that MOG-specific T-cells would show a large degree of overlap in the postimmune periphery and CNS, but non-specific cells that are not activated with immunization would show lower degree of overlap (Figure 6-1D).

At a population level, our data fail to identify a substantial role for interconversion as a means of locally generating Treg cells in the CNS. First, limited overlap of TCR used by Tconv and Treg cells in CNS and spleen was observed. And comparison of shared CDR3 amino acid sequences for each cohort demonstrated that the resemblance of repertoire among a single cell class (Treg or Tconv) across different organs (spleen and CNS) was greater than among different classes of cells within an organ. This result suggests that a distinct TCR repertoire is used by Tconv and Treg cells. Second, the frequencies of shared CDR3 among unique TCR sequences in preimmune splenocytes (expt 1: 3.5\%; expt 2: 6.4\%) was only modestly lower than that in postimmune splenocytes (expt 3: 10.1\%, expt 4: 6.6\%). The difference between postimmune splenocytes and CNS-infiltrating cells (expt 3: 11\%, expt 4: 14.7\%) was likewise small. These trends may be consistent with limited interconversion between Treg and Tconv cell lineages, but indicates that EAE does not promote substantial interconversion. It is correlated with the finding from NOD mice in which the encounter of cognate antigen in pancreas does not induce Tconv to Treg cell conversion (Wong et al., 2007a). Third, the heavy skewing of the minority of expressed by both Treg and Tconv TCR toward a single cell type argues against extensive plasticity in Foxp3 expression during EAE. Fourth, the acidic/aliphatic variation we observed in position 4 of MOG-specific TCR robustly discriminates between MOG-specific Treg TCR and Tconv TCR; implying auto-antigen specific Treg and Tconv populations are ontogenically distinct. Therefore, our results showed limited interconversion between Treg and Tconv cells both in spleen and CNS during MOG-EAE, and support the third model that inflammatory environment does not promote substantial interconversion between Treg and Tconv populations either in the periphery or the CNS (Figure 6-1C). 


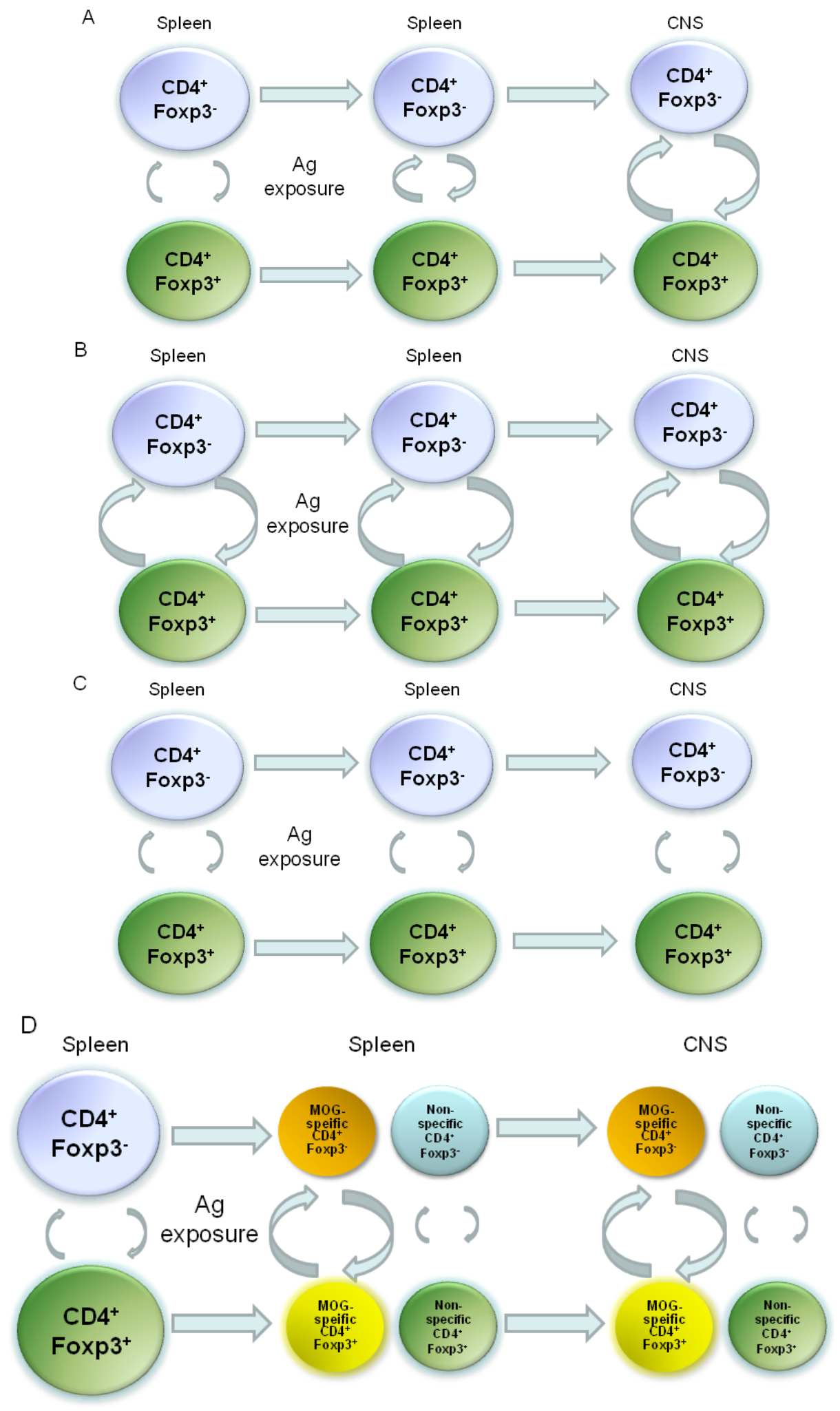

Figure 6-1. Models of interconversion between Tconv and Treg cells in MOG-EAE. 


\subsubsection{Limitations of the TCR Repertoire Analysis}

Although our data argue against interconversion, we must point out the limitations of experimental strategy. First, the sequence analysis was performed in two independent experiments. Although similar results were found, it is worthwhile to repeat it multiple times to further confirm the result. Second, the Tconv and Treg populations were separated by flow cytometry, and the sorting process can introduce a low level of error. Even though the purity of cell population was as high as $98 \%, 2 \%$ of cells could come from contamination and because of the larger number of Tconv cells, Tconv cells are more likely misplaced into Treg population, which may potentially exaggerate the overlap. Third, we acquired all the TCR sequence information from isolated cDNA instead of from single T-cell clones. This may introduce bias if differenT-cells express different amounts of TCR. However, in regards to this it is important to note that our conclusions were identical based on analyses of unique TCR rather than total TCR isolates. We also had to pool 5-6 mice together to harvest enough brain Treg cells for PCR. Therefore representation of TCR from different mice may differ and this could skew the repertoire assessments if they had different quantities of infiltrating T-cells. This limitation may lead to decreased similarity between the two populations, and an underestimation of the degree of overlap. However, we observed a dramatically increased overlap of single cell types across organs compared with differenT-cell types within one organ ( $\sim 30 \%$ versus $\sim 10 \%)$, and the result was reproducible, suggesting that skewing from pooling of organs did not alter our conclusions.

Another difficulty in whole repertoire analyses is that all TCR are analyzed, including cells that are not specific to $\mathrm{MOG}_{35-55}$. Tetrameric $\mathrm{pMHC}-\mathrm{MOG}$ complex staining may be a better technique to directly sort out TCRs with high-affinity to $\mathrm{MOG}_{35-55}$. However, autoreactive T-cells, including MOG-specific T-cells have low affinity, and the MOG-A $\mathrm{A}^{\mathrm{b}}$ tetramer only stains a minority of these cells. We attempted to stain 1MOG244.2 and other T-cells with this tetramer, but signal was not adequate for analyses (R. Alli and T.L.G., unpublished observations). We got around this problem by analyzing specific TCR for MOG reactivity. When we characterized the properties of selected TCRs in vitro, $100 \%$ of Tconv and $50 \%$ of Treg TCR from cells infiltrating the CNS were MOG-specific, much higher than the published data of about $10 \%$ of MOGspecific CNS-infiltrating T-cells using a MOG-A ${ }^{\mathrm{b}}$ tetramer (Korn et al., 2007). This may be due to that study's inability to detect low affinity but pathogenic TCRs, or our repertoire analyses only including high frequent TCR. It is possible that frequent T-cells may have a higher tendency toward MOG-specificity than low frequent T-cells. Nevertheless, despite its limitations, the approach we have used has a strong track record (Lin and Welsh, 1998; Pannetier et al., 1995; Sourdive et al., 1998), and has yielded new insights into the study of other repertoires.

\subsubsection{Future Studies}

To solve the problem generated by pooling cDNA, single-cell RT-PCR can be considered to acquire sequences of individual Tconv and Treg cells. This will also 
circumvent the problem of pooling mice to get enough T-cells from CNS, though it is technically more challenging and smaller numbers of sequences may be obtained. Alternatively, next-generation DNA sequencing can be used to confirm the limited conversion of Tconv and Treg cells (Freeman et al., 2009; Shendure and Ji, 2008). With the help of massive parallel DNA sequencing, one can interrogate the repertoire more thoroughly and rapidly. The whole analysis of $1 \mathrm{MOG} 244.2 \alpha$ EAE could be repeated at higher resolution with different primer sets, such as $\mathrm{V} \beta 14-\mathrm{C} \beta$ and $\mathrm{V} \beta 6-\mathrm{C} \beta$ to confirm our results. These experiments could also be performed at the different time point of disease - early onset or recovery phase, to see if there are temporal changes in interconversion between Foxp $3^{+}$and Foxp3 ${ }^{-}$cells. However, this scenario would seem unlikely considering our results indicating that interconversion between Foxp3 ${ }^{-}$and Foxp $3^{+}$cells is minimal. Additionally, the 1MOG244.2TCR $\alpha$ thymocytes repertoire has not been studied yet, comparing Treg and Tconv TCR in thymus, spleen and CNS will give more information and allow us to trace TCR clonotypes to the thymus.

Furthermore, experiments on the V $\beta 8.2$ repertoire of C57BL/6 mice with EAE can investigate the trend of interconversion in spleen and CNS in wild-type mice. It would be interesting to know whether the $\mathrm{D} / \mathrm{E}$ to $\mathrm{A} / \mathrm{V} / \mathrm{L}$ variation we found in retrogenic mice is also seen in wild-type mice with multiple $\alpha$ chains. Finally, repertoire analyses on peripheral T-cells from MS patients have not been performed, and such analyses may test whether results in EAE model is correlated with MS. One caveat here is that the cell surface markers for isolation of human Treg are imperfect, and isolation of pre Treg and Tconv populations in this setting will be a challenge.

\subsection{Segregation of Tconv and Treg Cells Based on CDR3 $\beta$ Sequence}

Analysis of selected receptors identified in our repertoire analyses provided insight into the composition of the auto-reactive Treg and Teff cell responses. MOGspecific Teff cells were highly enriched in the CNS of 1MOG244.2 $\alpha$ mice with EAE ( $100 \%$ of TCR). More interestingly, we identified a concentration of MOG-reactive TCR among the CNS-infiltrating $\mathrm{CD}^{+}{ }^{+} \mathrm{Foxp}^{+}$population (6/12 versus $1 / 8$ among $\mathrm{CD} 4^{+} \mathrm{Foxp}^{+}$splenocytes). This functionally confirms the presence of cognate autoantigen-reactive Treg cells in the CNS of mice with EAE at high frequency. Unlike Teff cells, which were uniformly MOG-specific, $50 \%$ of V $\beta 8.2^{+} \mathrm{J} \beta 2.7^{+}$Treg cell TCR tested did not recognize MOG. These cells may be bystanders or effectors specific for alternative antigens. Indeed, Treg cells need not to be specific for cognate autoantigen, and response toward tissue-specific or even tissue-unrestricted self antigens released during inflammation may potentially stimulate regulatory functions.

Among the MOG-specific T-cells, a length of 12 amino acids and a $\mathrm{G}$ at position 6 in the V $\beta 8.2^{+} \mathrm{J} \beta 2.7^{+} \mathrm{CDR} 3 \beta$ was highly correlated with MOG-specificity. Importantly, MOG-specific Teff cells during EAE were fully distinguished by a $\mathrm{D}$ or $\mathrm{E}$ at position 4, and Treg cells by an aliphatic residue, most commonly an A at the same site. Implicitly, Treg and Teff cells during autoimmunity arise from distinct population reservoirs, identifiable in 1MOG244.2 $\alpha$ retrogenic mouse EAE by the variation at residue 4. 
Additional structural criteria overlaid on this must be met for MOG-specificity. It is likely that these distinct populations arise from differences in the origin and selection of the T-cells, studies of developing thymocytes may help clarify this origin.

Recognition of the acidic versus aliphatic variation in CDR3 $\beta$ of Tconv and Treg cell was possible only by examining a narrow slice of the repertoire, one with a fixed TCR $\alpha$ and limited to V $\beta 8.2$ and J $\beta 2.7$. This selective repertoire analysis required the production and study of a manipulated mouse strain, the 1MOG244.2 $\alpha$ retrogenic mice. The consistent identification of specific public TCR within Treg or Tconv cell repertoires in these mice indicates consistency in T-cell differentiation patterns within them. However, the 1MOG244.2 $\alpha$ mice do use a $\mathrm{Tcra}^{-/-}$stem cell recipient, whose thymi developed in the absence of normal T-cell maturation. Further, premature expression of TCR $\alpha$ in transgenic or retrogenic systems, such as $1 \mathrm{MOG} 244.2 \alpha$ mice, can influence pT $\alpha$ signaling and decrease the efficiency of the DN to DP transition, and thus potentially altering the balance of different thymocyte subsets (Aifantis et al., 2006; Borowski et al., 2004; Huang and Kanagawa, 2004). We do not find evidence for these alterations influencing Treg cell lineage assignment. Grossly, proportions of Treg cells are normal in 1MOG244.2 $\alpha$ mice, and overlap among Treg and Tconv cell TCR is similar to that observed in other systems (Hsieh et al., 2006; Pacholczyk et al., 2006). More importantly, precursor cells retrogenic for specific Treg or Tconv cell TCR, when heavily diluted with wild-type bone marrow and transplanted into wild-type mice, show the same lineage predilection as in 1 MOG244.2 $\alpha$ mice. This suggests that TCR-driven Treg and Tconv cell lineage specification in $1 \mathrm{MOG} 244.2 \alpha$ mice is indicative of the potentials of the same TCR in normal thymi as well.

\subsection{Future Plans to Confirm the Segregation of Treg and Tconv Based on TCR Sequence}

If the hypothesis that the acidic versus aliphatic variation fundamentally influences T-cell lineage assignment is true, exchange of acidic to aliphatic residues or vica versa at position 4 may lead to T-cell lineage re-commitment. We have mutated a D or $\mathrm{E}$ at position 4 to an $\mathrm{A}$ in MOG-specific Tconv TCR, and an A, V, L at same position to a $\mathrm{D}$ in Treg TCR. The mutations were confirmed by sequencing. The idea is to use these TCR to repeat the experiment shown in Section 5.2.1. Specifically the TCR will be retrovirally transduced into HPCs from $\mathrm{CD} 45.2^{+} \mathrm{Rag}^{-/-}$mice, then transplant into sublethally irradiated congenic CD $45.1^{+}$mice after dilution with CD45. $1^{+}$HPCs. After 8 weeks, we will analyze Foxp3 expression among CD45.2 ${ }^{+} \mathrm{T}$-cell population, and the mice with original TCR will serve as control. If the hypothesis is correct, Foxp3 expression will be elevated in mice with Tconv TCR mutated to express an A at position 4 compared with mice containing original TCR. On the other hand, Foxp3 expression will be diminished in mixed chimera with Treg TCR mutated to express a D at position 4. 


\subsection{Affinity in Treg Responses}

\subsubsection{Models of Treg Repertoire Selection}

Treg cells have been alternatively described as recognizing self-antigens with a relative higher affinity than Tconv cells, having increased or moderate avidity for self, and overlapping in recognition characteristics with pathogenic auto-reactive T-cells. Experiments with TCR transgenic mice suggest that precursors develop into the Treg lineage when higher avidity interactions with selecting ligands were found (Apostolou et al., 2002; Jordan et al., 2001; Kawahata et al., 2002; Walker et al., 2003). Subsequent studies proposed a two-step model that TCR-dependent signals followed by TCRindependent IL-2 signaling and activation of Stat5 signals (Burchill et al., 2008; Lio and Hsieh, 2008; Liston and Rudensky, 2007). In this model, commitment of Treg cells is driven by higher affinity interactions between the TCR and MHC/self-peptide complexes, followed by accessory signals (IL-2, B7 and APC etc). Consequently, Tconv and Treg cells express different sets of TCRs that have different affinity to self-antigens (Figure 6-2A). However, the actual range of this "higher" affinity for self antigens has never been determined, especially in the polyclonal Treg repertoire. Possibly affinity or avidity alone is not an exclusive determinant of Treg lineage assignment. Even TCRs with weak affinities to self antigen may possibly be included in the Treg repertoire.

An alternative presumption is the role of TCR-affinity dependent signals may be complementary rather than dominant in Treg lineage selection. In this model, the commitment of Treg precursors occurs prior to thymic selection by TCR, and the TCRdependent and/or TCR-independent signals may further complete the Treg lineage commitment. Therefore the final Treg repertoire can be enriched in self-reactive and non-reactive TCRs, and the separation of Treg and Tconv cells is achieved by both TCRdependent and TCR-independent signals (Figure 6-2B). This model would explain several findings. First, Foxp3 expression can occur in pre-selected CD4 CD8thymocytes (Pennington et al., 2006). Second, Treg cells can recognize a wide range of foreign antigens and have a diverse repertoire. Third, increased overlap between Tconv and Treg cells was seen in "single-peptide" transgene mice, where all MHCII molecules are occupied by one peptide (Pacholczyk and Kern, 2008).

\subsubsection{Sensitivity and Fine Specificity of MOG-specific Treg and Tconv TCR}

Expression of high-affinity cognate antigen on thymic stroma promotes enhanced Treg cell differentiation for some TCR (Picca et al., 2006). MOG is expressed at low levels in the thymus (Delarasse et al., 2003). If MOG sensitivity guides Treg cell development, then according to the first (two-step) model introduced above, MOGspecific Treg should be more sensitive to MOG than specific Tconv cells. Overall, Treg cell TCR showed higher MOG sensitivity than did Teff cell TCR. However, the substantial overlap in Treg and Teff cell TCR sensitivity implied that this was not fundamental to their selection (Figure 5-3). Further, differential fine specificity for MOG 


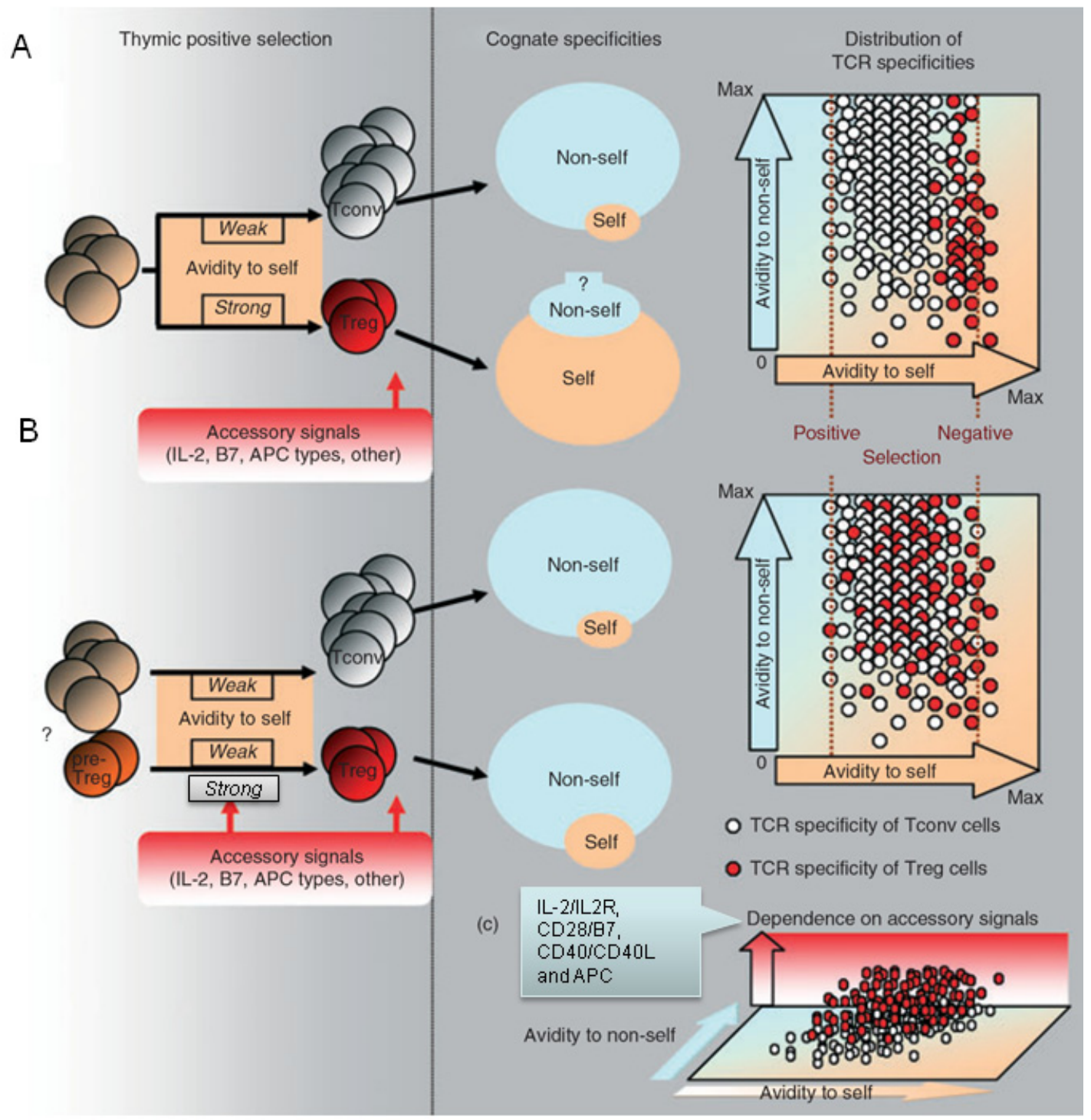

Figure 6-2. Generation of specificities of the Treg repertoire. Reprinted with permission. Pacholczyk, R., and Kern, J. (2008). The T-cell receptor repertoire of regulatory T-cells. Immunology 125, 450-458. 
was not identified in Treg and Teff cell TCR.

There are several explanations for the similar MOG response yet different and conserved CDR3 features of Treg and Teff cell TCR. One is that lineage selection for the corresponding cell types is not MOG-dependent, but rather that an alternative $\mathrm{Ag}(\mathrm{s})$ promotes selection, which we will further discuss in Chapter 6.6.1. Second, the antigen sensitivity we measured in the 4G4 system is not the actual affinity to MOG-peptide. Theoretically it should be correlated with affinity because of identical CD4 and TCR expression on the 4G4 cells, essentially these cells only differed in their CDR3 $\beta$. However, direct measurement of affinity should be considered to confirm the results from the CD4.4G4 cells, such as by Surface Plasmon Resonance (SPR). A third possibility is that Treg TCR do not necessarily show higher affinity to self-antigen, as described above (Figure 6-2B). That would explain the observation of the low sensitivity of some Treg TCR (\#9 and \#52) in our experiment, and overlapping sensitivity to MOG-peptide among Treg and Tconv TCRs. In addition, when Treg TCRs (\#1, \#9 and \#18) from different "sensitivity windows" were tested for giving rise to Treg cells in mixed-BM chimeric mice, Treg cells developed with all three TCRs, up to $\sim 30 \%$ at very low frequencies. No deletion was seen in $\# 1$ receptor, which showed highest sensitivity and maximal response in CD4.4G4 cells.

It will also be important to determine how the sensitivity or affinity of Treg TCR influences their protective abilities. To address this, further in vivo studies should be performed on the Treg TCRs. To determine their actual function in EAE, we will generate mixed-BM chimeras with low or high-sensitivity receptors, and challenge them with MOG peptide, to see whether there are differences in the ability of these receptors to protect mice from EAE.

\subsection{Antigen Specificity in Regulatory T-cell Responses}

\subsubsection{Does MOG-specific T-cell Cross-react with Alternative Self-antigen Which Mediates Thymic Selection?}

In order to mount a rapid and efficient immune response against invading pathogens, TCRs have evolved a high level of antigen cross-reactivity and recognize multiple different peptides bound to MHC molecules. Cross-reactivity is necessary for $\mathrm{T}$-cell development and is critical given the fixed size of the T-cell repertoire relative to the vast universe of potential antigens (Bhardwaj et al., 1993; Evavold et al., 1995; Mason, 1998; Maverakis et al., 2001; Wilson et al., 2004; Wucherpfennig and Strominger, 1995). Mason et al. estimated that each T-cell is capable of reacting with $\sim 10^{6}$ different MHC-associated peptide epitopes (Mason, 1998), and Hiemstra et al. further confirmed the estimation through deduction of effective concentrations of agonist ligands in a peptide-"gemisch" (Hiemstra et al., 1998). Although the possible antigens are non-self peptides, based on these estimates it must be considered that any particular TCR will likely be specific for more than one self-antigen. Molecular mimicry and cross 
reactivity is frequently found in autoimmunity. An elegant example is that mice developed EAE after immunization with peptide of hepatitis B virus, which shared a sequence of six consecutive amino acids in common with MBP (Wucherpfennig and Strominger, 1995). Subsequent studies showed that only a few critical amino acids serving as contact residues were needed to be shared by two antigenic peptides in order to elicit cross-reactivity (Evavold et al., 1993; Kersh and Allen, 1996; Madrenas et al., 1995).

In one study, MOG-specific 2D2 TCR transgenic mice developed EAE in the absence of MOG because 2D2 T-cells cross react with neurofilament-M (NF-M $18-30$, TETRSSFSRVSGS), which shares crucial contact amino acids with $\mathrm{MOG}_{35-55}$ (MEVGWYRSPFSRVVHLYRNGK) (Krishnamoorthy et al., 2009). We hypothesized that the Treg cell identified here could be selected by NF-M $\mathrm{M}_{18-30}$ in thymus originally, and could cross react with $\mathrm{MOG}_{35-55}$. As a first step to test this hypothesis, we transduced the original MOG-specific TCR and their counterpart mutations into CD4.4G4 cells, then examined their responsiveness to NF-M $18-30$ and $\mathrm{MOG}_{35-55}$ by IL-2 production. If NF$\mathrm{M}_{18-30}$ is responsible for Treg selection, Treg TCRs recognize NF-M and may have higher sensitivity than Tconv TCR. Further, the D/E versus A/V/L variation is responsible for T-cell lineage assignment, mutation in Treg TCR should lead to the decreased response to NF-M $18-30$, whereas mutation in Tconv TCR may enhance their recognition to NF-M $18-30$. So far pilot experiments were performed on 12 receptors. 4 out of 6 mutated Tconv TCR showed increased sensitivity, and 4 out of 6 mutated Treg TCR showed decreased sensitivity to NF-M. We will confirm this result by testing more receptors.

\subsubsection{What Do Non-MOG-specific Treg TCR Recognize?}

Epitope spreading is when the specificity of T and B-cells in the immune response spreads or switches from the initiating epitope to other epitopes, including self epitopes, (Vanderlugt and Miller, 2002). This phenomenon has been observed in PLP $_{139-151}$ induced EAE in the SJL mouse, where after priming SJL mice with PLP P $_{139-151}$, the immune response switches from anti-PLP ${ }_{139-151}$ to anti-PLP ${ }_{178-191}$ and anti-MBP $84-104$ during the course of EAE (McRae et al., 1995; Vanderlugt et al., 2000). These findings were based on total $\mathrm{CD}^{+}{ }^{+} \mathrm{T}$-cells, mainly effector T-cells. In MOG-EAE epitope spread has not been seen in the Teff response. Whether epitope spreading exists in Treg cells in MOG-EAE, however, remains uncertain.

In the CNS of $1 \mathrm{MOG} 244.2 \alpha$ mice, we identified six receptors (50\%) from the Treg population that were not MOG-specific. Kuchroo et al. showed that 7\% and 34\% CNS-infiltrating Treg are MOG tetramer-positive in the peak and recovery phase, respectively, and concluded that the rest of Treg are bystanders (Korn et al., 2007). Why do these "bystander" Treg cells accumulate in CNS? Do they recognize other selfantigen(s) because of epitope spreading? To answer this question, we can test whether these receptors react with $\mathrm{NF}-\mathrm{M}_{18-30}$ using CD4.4G4 hybridomas. 
The second question is whether these non-MOG-specific Treg cells play a role in EAE protection. We tried to generate retrogenic mice expressing a single TCR, but the number of monoclonal Treg cells acquired from single TCR-retrogenic mice was very low presumably because of niche saturation. We also tried to convert Tconv cells isolated from these retrogenic mice into Treg with TGF- $\beta$ and high dose of IL-2 and injected the induced Treg cells into $\mathrm{Rag}^{-/-}$mice, to test EAE protection. Unfortunately, it was much more difficult to expand small numbers of clonal cell from retrogenic mice than Tconv cells from wild-type, and we also found the converting efficiency was receptor dependent and often poor. Further studies will be needed to optimize this. Hsieh's group created mixed-bone marrow chimeras with varying ratios of polyclonal progenitor cells and progenitor cells transgenic for Treg cell-derived TCRs. We will also generate similar mixed-bone marrow chimeras with retrovirally transduced HPCs expressing "bystander Treg TCR" using HPCs are from Foxp3-GFP x Rag ${ }^{-/-}$mice. After 8 weeks transplantation, we will examine Treg with transduced TCR in peripheral blood by GFP. Positive mice will be challenged with MOG peptide and EAE will be followed to determine if the retrogenic Treg diminish disease severity.

In summary, we assessed Treg and Tconv repertoires in MOG-EAE. Under inflammatory conditions, the Tconv used a repertoire distinct from that of Treg, though the repertoires overlapped. The extent of this overlap was not significantly increased with immunization. Both MOG-specific Treg and Tconv with distinct repertoires are concentrated within the CNS during EAE, though infiltrating Treg seemingly possess a broader spectrum of antigen specificities. Examination of MOG-specific Treg and Teff cell TCR with a fixed TCR $\alpha$ and restricted TCR $\beta$ usage failed to find evidence for sensitivity or fine specificity for MOG influencing Treg versus Tconv cell lineage assignment, but did reveal lineage segregation based on conserved CDR3 features. Our results imply that ontogenically distinct Treg and Tconv cell repertoires during MOGEAE and argue against more than limited plasticity between Treg and Tconv cells during the autoinflammatory response. 


\section{LIST OF REFERENCES}

Aebischer, T., Oehen, S., and Hengartner, H. (1990). Preferential usage of V alpha 4 and $\mathrm{V}$ beta $10 \mathrm{~T}$-cell receptor genes by lymphocytic choriomeningitis virus glycoproteinspecific H-2Db-restricted cytotoxic T-cells. Eur J Immunol 20, 523-531.

Aifantis, I., Mandal, M., Sawai, K., Ferrando, A., and Vilimas, T. (2006). Regulation of T-cell progenitor survival and cell-cycle entry by the pre-T-cell receptor. Immunol Rev 209, 159-169.

Alli, R., Nguyen, P., and Geiger, T.L. (2008). Retrogenic modeling of experimental allergic encephalomyelitis associates T-cell frequency but not TCR functional affinity with pathogenicity. J Immunol 181, 136-145.

Almeida, A.R., Legrand, N., Papiernik, M., and Freitas, A.A. (2002). Homeostasis of peripheral CD4+ T-cells: IL-2R alpha and IL-2 shape a population of regulatory cells that controls CD4+ T-cell numbers. J Immunol 169, 4850-4860.

Alt, C., Laschinger, M., and Engelhardt, B. (2002). Functional expression of the lymphoid chemokines CCL19 (ELC) and CCL 21 (SLC) at the blood-brain barrier suggests their involvement in G-protein-dependent lymphocyte recruitment into the central nervous system during experimental autoimmune encephalomyelitis. Eur J Immunol 32, 2133-2144.

Andersson, J., Stefanova, I., Stephens, G.L., and Shevach, E.M. (2007). CD4+CD25+ regulatory T-cells are activated in vivo by recognition of self. Int Immunol 19, 557-566.

Apostolou, I., Sarukhan, A., Klein, L., and von Boehmer, H. (2002). Origin of regulatory T-cells with known specificity for antigen. Nat Immunol 3, 756-763.

Apostolou, I. and von Boehmer, H. (2004). In vivo instruction of suppressor commitment in naive T-cells. J Exp Med 199, 1401-1408.

Asano, M., Toda, M., Sakaguchi, N., and Sakaguchi, S. (1996). Autoimmune disease as a consequence of developmental abnormality of a T-cell subpopulation. J Exp Med 184, 387-396.

Asseman, C., Mauze, S., Leach, M.W., Coffman, R.L., and Powrie, F. (1999). An essential role for interleukin 10 in the function of regulatory T-cells that inhibit intestinal inflammation. J Exp Med 190, 995-1004.

Bailey, S.L., Schreiner, B., McMahon, E.J., and Miller, S.D. (2007). CNS myeloid DCs presenting endogenous myelin peptides 'preferentially' polarize CD4+ T(H)-17 cells in relapsing EAE. Nat Immunol 8, 172-180. 
Baker, F.J., Lee, M., Chien, Y.H., and Davis, M.M. (2002). Restricted islet-cell reactive T-cell repertoire of early pancreatic islet infiltrates in NOD mice. Proc Natl Acad Sci U S A 99, 9374-9379.

Baldwin, T.A., Sandau, M.M., Jameson, S.C., and Hogquist, K.A. (2005). The timing of TCR alpha expression critically influences T-cell development and selection. J Exp Med 202, 111-121.

Bautista, J.L., Lio, C.W., Lathrop, S.K., Forbush, K., Liang, Y., Luo, J., Rudensky, A.Y., and Hsieh, C.S. (2009). Intraclonal competition limits the fate determination of regulatory T-cells in the thymus. Nat Immunol 10, 610-617.

Ben-Nun, A., Kerlero de Rosbo, N., Kaushansky, N., Eisenstein, M., Cohen, L., Kaye, J.F., and Mendel, I. (2006). Anatomy of T-cell autoimmunity to myelin oligodendrocyte glycoprotein (MOG): prime role of MOG44F in selection and control of MOG-reactive T-cells in H-2b mice. Eur J Immunol 36, 478-493.

Bender, J., Mitchell, T., Kappler, J., and Marrack, P. (1999). CD4+ T-cell division in irradiated mice requires peptides distinct from those responsible for thymic selection. $\mathrm{J}$ Exp Med 190, 367-374.

Bensinger, S.J., Bandeira, A., Jordan, M.S., Caton, A.J., and Laufer, T.M. (2001). Major histocompatibility complex class II-positive cortical epithelium mediates the selection of CD4(+)25(+) immunoregulatory T-cells. J Exp Med 194, 427-438.

Benson, M.J., Pino-Lagos, K., Rosemblatt, M., and Noelle, R.J. (2007). All-trans retinoic acid mediates enhanced $\mathrm{T}$ reg cell growth, differentiation, and gut homing in the face of high levels of co-stimulation. J Exp Med 204, 1765-1774.

Bettelli, E., Carrier, Y., Gao, W., Korn, T., Strom, T.B., Oukka, M., Weiner, H.L., and Kuchroo, V.K. (2006). Reciprocal developmental pathways for the generation of pathogenic effector TH17 and regulatory T-cells. Nature 441, 235-238.

Bhardwaj, V., Kumar, V., Geysen, H.M., and Sercarz, E.E. (1993). Degenerate recognition of a dissimilar antigenic peptide by myelin basic protein-reactive T-cells. Implications for thymic education and autoimmunity. J Immunol 151, 5000-5010.

Bonasio, R., Scimone, M.L., Schaerli, P., Grabie, N., Lichtman, A.H., and von Andrian, U.H. (2006). Clonal deletion of thymocytes by circulating dendritic cells homing to the thymus. Nat Immunol 7, 1092-1100.

Borowski, C., Li, X., Aifantis, I., Gounari, F., and von Boehmer, H. (2004). PreTCRalpha and TCRalpha are not interchangeable partners of TCRbeta during Tlymphocyte development. J Exp Med 199, 607-615. 
Brunkow, M.E., Jeffery, E.W., Hjerrild, K.A., Paeper, B., Clark, L.B., Yasayko, S.A., Wilkinson, J.E., Galas, D., Ziegler, S.F., and Ramsdell, F. (2001). Disruption of a new forkhead/winged-helix protein, scurfin, results in the fatal lymphoproliferative disorder of the scurfy mouse. Nat Genet $27,68-73$.

Burchill, M.A., Yang, J., Vang, K.B., Moon, J.J., Chu, H.H., Lio, C.W., Vegoe, A.L., Hsieh, C.S., Jenkins, M.K., and Farrar, M.A. (2008). Linked T-cell receptor and cytokine signaling govern the development of the regulatory T-cell repertoire. Immunity 28, 112121.

Callan, M.F., Steven, N., Krausa, P., Wilson, J.D., Moss, P.A., Gillespie, G.M., Bell, J.I., Rickinson, A.B., and McMichael, A.J. (1996). Large clonal expansions of CD8+ T-cells in acute infectious mononucleosis. Nat Med 2, 906-911.

Carter, J.D., Calabrese, G.M., Naganuma, M., and Lorenz, U. (2005). Deficiency of the Src homology region 2 domain-containing phosphatase 1 (SHP-1) causes enrichment of CD4+CD25+ regulatory T-cells. J Immunol 174, 6627-6638.

Casrouge, A., Beaudoing, E., Dalle, S., Pannetier, C., Kanellopoulos, J., and Kourilsky, P. (2000). Size estimate of the alpha beta TCR repertoire of naive mouse splenocytes. J Immunol 164, 5782-5787.

Cederbom, L., Hall, H., and Ivars, F. (2000). CD4+CD25+ regulatory T-cells downregulate co-stimulatory molecules on antigen-presenting cells. Eur J Immunol 30, 15381543.

Chang, T.T., Jabs, C., Sobel, R.A., Kuchroo, V.K., and Sharpe, A.H. (1999). Studies in B7-deficient mice reveal a critical role for B7 costimulation in both induction and effector phases of experimental autoimmune encephalomyelitis. J Exp Med 190, 733-740.

Chen, W., Jin, W., Hardegen, N., Lei, K.J., Li, L., Marinos, N., McGrady, G., and Wahl, S.M. (2003). Conversion of peripheral CD4+CD25- naive T-cells to CD4+CD25+ regulatory T-cells by TGF-beta induction of transcription factor Foxp3. J Exp Med 198, 1875-1886.

Cobbold, S.P., Castejon, R., Adams, E., Zelenika, D., Graca, L., Humm, S., and Waldmann, H. (2004). Induction of FoxP3+ regulatory T-cells in the periphery of T-cell receptor transgenic mice tolerized to transplants. J Immunol 172, 6003-6010.

Collison, L.W., Pillai, M.R., Chaturvedi, V., and Vignali, D.A. (2009). Regulatory T-cell suppression is potentiated by target T-cells in a cell contact, IL-35- and IL-10-dependent manner. J Immunol 182, 6121-6128.

Collison, L.W., Workman, C.J., Kuo, T.T., Boyd, K., Wang, Y., Vignali, K.M., Cross, R., Sehy, D., Blumberg, R.S., and Vignali, D.A. (2007). The inhibitory cytokine IL-35 contributes to regulatory T-cell function. Nature 450, 566-569. 
Coombes, J.L., Siddiqui, K.R., Arancibia-Carcamo, C.V., Hall, J., Sun, C.M., Belkaid, Y., and Powrie, F. (2007). A functionally specialized population of mucosal CD103+ DCs induces Foxp3+ regulatory T-cells via a TGF-beta and retinoic acid-dependent mechanism. J Exp Med 204, 1757-1764.

Correia-Neves, M., Waltzinger, C., Mathis, D., and Benoist, C. (2001). The shaping of the T-cell repertoire. Immunity 14, 21-32.

Curotto de Lafaille, M.A., Lino, A.C., Kutchukhidze, N., and Lafaille, J.J. (2004). CD25T-cells generate CD25+Foxp3+ regulatory T-cells by peripheral expansion. J Immunol $173,7259-7268$.

D'Cruz, L.M. and Klein, L. (2005). Development and function of agonist-induced $\mathrm{CD} 25+\mathrm{Foxp} 3+$ regulatory T-cells in the absence of interleukin 2 signaling. Nat Immunol $6,1152-1159$.

Davidson, T.S., DiPaolo, R.J., Andersson, J., and Shevach, E.M. (2007). Cutting Edge: IL-2 is essential for TGF-beta-mediated induction of Foxp3+ T regulatory cells. J Immunol 178, 4022-4026.

Davis, M.M. and Bjorkman, P.J. (1988). T-cell antigen receptor genes and T-cell recognition. Nature 334, 395-402.

Davis, M.M., Boniface, J.J., Reich, Z., Lyons, D., Hampl, J., Arden, B., and Chien, Y. (1998). Ligand recognition by alpha beta T-cell receptors. Annu Rev Immunol 16, 523544.

Delarasse, C., Daubas, P., Mars, L.T., Vizler, C., Litzenburger, T., Iglesias, A., Bauer, J., Della Gaspera, B., Schubart, A., Decker, L., et al. (2003). Myelin/oligodendrocyte glycoprotein-deficient (MOG-deficient) mice reveal lack of immune tolerance to MOG in wild-type mice. J Clin Invest 112, 544-553.

Dembic, Z., Haas, W., Weiss, S., McCubrey, J., Kiefer, H., von Boehmer, H., and Steinmetz, M. (1986). Transfer of specificity by murine alpha and beta T-cell receptor genes. Nature 320, 232-238.

Engelhardt, B. (2006). Molecular mechanisms involved in T-cell migration across the blood-brain barrier. J Neural Transm 113, 477-485.

Engelhardt, B., Laschinger, M., Schulz, M., Samulowitz, U., Vestweber, D., and Hoch, G. (1998). The development of experimental autoimmune encephalomyelitis in the mouse requires alpha4-integrin but not alpha4beta7-integrin. J Clin Invest 102, 2096-2105.

Ernst, B., Lee, D.S., Chang, J.M., Sprent, J., and Surh, C.D. (1999). The peptide ligands mediating positive selection in the thymus control T-cell survival and homeostatic proliferation in the periphery. Immunity 11, 173-181. 
Evavold, B.D., Sloan-Lancaster, J., and Allen, P.M. (1993). Tickling the TCR: selective T-cell functions stimulated by altered peptide ligands. Immunol Today 14, 602-609.

Evavold, B.D., Sloan-Lancaster, J., Wilson, K.J., Rothbard, J.B., and Allen, P.M. (1995). Specific T-cell recognition of minimally homologous peptides: evidence for multiple endogenous ligands. Immunity 2, 655-663.

Fahlen, L., Read, S., Gorelik, L., Hurst, S.D., Coffman, R.L., Flavell, R.A., and Powrie, F. (2005). T-cells that cannot respond to TGF-beta escape control by CD4(+)CD25(+) regulatory T-cells. J Exp Med 201, 737-746.

Fazilleau, N., Delarasse, C., Sweenie, C.H., Anderton, S.M., Fillatreau, S., Lemonnier, F.A., Pham-Dinh, D., and Kanellopoulos, J.M. (2006). Persistence of autoreactive myelin oligodendrocyte glycoprotein (MOG)-specific T-cell repertoires in MOG-expressing mice. Eur J Immunol 36, 533-543.

Fontenot, J.D., Gavin, M.A., and Rudensky, A.Y. (2003). Foxp3 programs the development and function of CD4+CD25+ regulatory T-cells. Nat Immunol 4, 330-336.

Fontenot, J.D., Rasmussen, J.P., Gavin, M.A., and Rudensky, A.Y. (2005a). A function for interleukin 2 in Foxp3-expressing regulatory T-cells. Nat Immunol 6, 1142-1151.

Fontenot, J.D., Rasmussen, J.P., Williams, L.M., Dooley, J.L., Farr, A.G., and Rudensky, A.Y. (2005b). Regulatory T-cell lineage specification by the forkhead transcription factor foxp3. Immunity 22, 329-341.

Fowell, D. and Mason, D. (1993). Evidence that the T-cell repertoire of normal rats contains cells with the potential to cause diabetes. Characterization of the CD4+ T-cell subset that inhibits this autoimmune potential. J Exp Med 177, 627-636.

Freeman, J.D., Warren, R.L., Webb, J.R., Nelson, B.H., and Holt, R.A. (2009). Profiling the T-cell receptor beta-chain repertoire by massively parallel sequencing. Genome Res $19,1817-1824$.

Fujishima, M., Hirokawa, M., Fujishima, N., and Sawada, K. (2005). TCRalphabeta repertoire diversity of human naturally occurring $\mathrm{CD} 4+\mathrm{CD} 25+$ regulatory T-cells. Immunol Lett 99, 193-197.

Furmanski, A.L., Bartok, I., Chai, J.G., Singh, Y., Ferreira, C., Scott, D., Holland, S.J., Bourdeaux, C., Crompton, T., and Dyson, J. Peptide-specific, TCR-alpha-driven, coreceptor-independent negative selection in TCR alpha-chain transgenic mice. J Immunol 184, 650-657.

Garin, M.I., Chu, C.C., Golshayan, D., Cernuda-Morollon, E., Wait, R., and Lechler, R.I. (2007). Galectin-1: a key effector of regulation mediated by CD4+CD25+ T-cells. Blood 109, 2058-2065. 
Gartner, F., Alt, F.W., Monroe, R., Chu, M., Sleckman, B.P., Davidson, L., and Swat, W. (1999). Immature thymocytes employ distinct signaling pathways for allelic exclusion versus differentiation and expansion. Immunity 10, 537-546.

Geiger, T.L., Nguyen, P., Leitenberg, D., and Flavell, R.A. (2001). Integrated src kinase and costimulatory activity enhances signal transduction through single-chain chimeric receptors in T-lymphocytes. Blood 98, 2364-2371.

Gondek, D.C., Lu, L.F., Quezada, S.A., Sakaguchi, S., and Noelle, R.J. (2005). Cutting Edge: contact-mediated suppression by $\mathrm{CD} 4+\mathrm{CD} 25+$ regulatory cells involves a granzyme B-dependent, perforin-independent mechanism. J Immunol 174, 1783-1786.

Grajewski, R.S., Silver, P.B., Agarwal, R.K., Su, S.B., Chan, C.C., Liou, G.I., and Caspi, R.R. (2006). Endogenous IRBP can be dispensable for generation of natural $\mathrm{CD} 4+\mathrm{CD} 25+$ regulatory T-cells that protect from IRBP-induced retinal autoimmunity. $\mathrm{J}$ Exp Med 203, 851-856.

Grossman, W.J., Verbsky, J.W., Barchet, W., Colonna, M., Atkinson, J.P., and Ley, T.J. (2004). Human T regulatory cells can use the perforin pathway to cause autologous targe T-cell death. Immunity 21, 589-601.

Hagenbeek, T.J., Naspetti, M., Malergue, F., Garcon, F., Nunes, J.A., Cleutjens, K.B., Trapman, J., Krimpenfort, P., and Spits, H. (2004). The loss of PTEN allows TCR alphabeta lineage thymocytes to bypass IL-7 and Pre-TCR-mediated signaling. J Exp Med 200, 883-894.

Hawrylowicz, C.M. and O'Garra, A. (2005). Potential role of interleukin-10-secreting regulatory T-cells in allergy and asthma. Nat Rev Immunol 5, 271-283.

Hiemstra, H.S., van Veelen, P.A., Schloot, N.C., Geluk, A., van Meijgaarden, K.E., Willemen, S.J., Leunissen, J.A., Benckhuijsen, W.E., Amons, R., de Vries, R.R., et al. (1998). Definition of natural T-cell antigens with mimicry epitopes obtained from dedicated synthetic peptide libraries. J Immunol 161, 4078-4082.

Hoffmann, P., Boeld, T.J., Eder, R., Huehn, J., Floess, S., Wieczorek, G., Olek, S., Dietmaier, W., Andreesen, R., and Edinger, M. (2009). Loss of FOXP3 expression in natural human $\mathrm{CD} 4+\mathrm{CD} 25+$ regulatory $\mathrm{T}$-cells upon repetitive in vitro stimulation. Eur $\mathrm{J}$ Immunol 39, 1088-1097.

Holst, J., Szymczak-Workman, A.L., Vignali, K.M., Burton, A.R., Workman, C.J., and Vignali, D.A. (2006a). Generation of T-cell receptor retrogenic mice. Nat Protoc 1, 406417.

Holst, J., Vignali, K.M., Burton, A.R., and Vignali, D.A. (2006b). Rapid analysis of Tcell selection in vivo using T-cell-receptor retrogenic mice. Nat Methods 3, 191-197. 
Hori, S., Haury, M., Coutinho, A., and Demengeot, J. (2002). Specificity requirements for selection and effector functions of CD25+4+ regulatory T-cells in anti-myelin basic protein T-cell receptor transgenic mice. Proc Natl Acad Sci U S A 99, 8213-8218.

Hori, S., Nomura, T., and Sakaguchi, S. (2003). Control of regulatory T-cell development by the transcription factor Foxp3. Science 299, 1057-1061.

Houot, R., Perrot, I., Garcia, E., Durand, I., and Lebecque, S. (2006). Human $\mathrm{CD} 4+\mathrm{CD} 25$ high regulatory T-cells modulate myeloid but not plasmacytoid dendritic cells activation. J Immunol 176, 5293-5298.

Hsieh, C.S., Liang, Y., Tyznik, A.J., Self, S.G., Liggitt, D., and Rudensky, A.Y. (2004). Recognition of the peripheral self by naturally arising CD25+ CD4+ T-cell receptors. Immunity 21, 267-277.

Hsieh, C.S., Zheng, Y., Liang, Y., Fontenot, J.D., and Rudensky, A.Y. (2006). An intersection between the self-reactive regulatory and nonregulatory T-cell receptor repertoires. Nat Immunol 7, 401-410.

Huang, C.T., Workman, C.J., Flies, D., Pan, X., Marson, A.L., Zhou, G., Hipkiss, E.L., Ravi, S., Kowalski, J., Levitsky, H.I., et al. (2004). Role of LAG-3 in regulatory T-cells. Immunity 21, 503-513.

Huang, C.Y. and Kanagawa, O. (2004). Impact of early expression of TCR alpha chain on thymocyte development. Eur J Immunol 34, 1532-1541.

Huter, E.N., Stummvoll, G.H., DiPaolo, R.J., Glass, D.D., and Shevach, E.M. (2008). Cutting Edge: antigen-specific TGF beta-induced regulatory T-cells suppress Th17mediated autoimmune disease. J Immunol 181, 8209-8213.

Iritani, B.M., Alberola-Ila, J., Forbush, K.A., and Perimutter, R.M. (1999). Distinct signals mediate maturation and allelic exclusion in lymphocyte progenitors. Immunity 10, 713-722.

Itoh, M., Takahashi, T., Sakaguchi, N., Kuniyasu, Y., Shimizu, J., Otsuka, F., and Sakaguchi, S. (1999). Thymus and autoimmunity: production of CD25+CD4+ naturally anergic and suppressive T-cells as a key function of the thymus in maintaining immunologic self-tolerance. J Immunol 162, 5317-5326.

Joetham, A., Takeda, K., Taube, C., Miyahara, N., Matsubara, S., Koya, T., Rha, Y.H., Dakhama, A., and Gelfand, E.W. (2007). Naturally occurring lung CD4(+)CD25(+) Tcell regulation of airway allergic responses depends on IL-10 induction of TGF-beta. $\mathrm{J}$ Immunol 178, 1433-1442. 
Jordan, M.S., Boesteanu, A., Reed, A.J., Petrone, A.L., Holenbeck, A.E., Lerman, M.A., Naji, A., and Caton, A.J. (2001). Thymic selection of CD4+CD25+ regulatory T-cells induced by an agonist self-peptide. Nat Immunol 2, 301-306.

Kasow, K.A., Chen, X., Knowles, J., Wichlan, D., Handgretinger, R., and Riberdy, J.M. (2004). Human CD4+CD25+ regulatory T-cells share equally complex and comparable repertoires with CD4+CD25- counterparts. J Immunol 172, 6123-6128.

Kawahata, K., Misaki, Y., Yamauchi, M., Tsunekawa, S., Setoguchi, K., Miyazaki, J., and Yamamoto, K. (2002). Generation of CD4(+)CD25(+) regulatory T-cells from autoreactive T-cells simultaneously with their negative selection in the thymus and from nonautoreactive T-cells by endogenous TCR expression. J Immunol 168, 4399-4405.

Kedzierska, K., Turner, S.J., and Doherty, P.C. (2004). Conserved T-cell receptor usage in primary and recall responses to an immunodominant influenza virus nucleoprotein epitope. Proc Natl Acad Sci U S A 101, 4942-4947.

Kersh, G.J. and Allen, P.M. (1996). Essential flexibility in the T-cell recognition of antigen. Nature 380, 495-498.

Khattri, R., Cox, T., Yasayko, S.A., and Ramsdell, F. (2003). An essential role for Scurfin in CD4+CD25+ T regulatory cells. Nat Immunol 4, 337-342.

Kim, J.M. and Rudensky, A. (2006). The role of the transcription factor Foxp3 in the development of regulatory T-cells. Immunol Rev 212, 86-98.

Knoechel, B., Lohr, J., Kahn, E., Bluestone, J.A., and Abbas, A.K. (2005). Sequential development of interleukin 2-dependent effector and regulatory T-cells in response to endogenous systemic antigen. J Exp Med 202, 1375-1386.

Kohm, A.P., Carpentier, P.A., Anger, H.A., and Miller, S.D. (2002). Cutting Edge: CD4+CD25+ regulatory T-cells suppress antigen-specific autoreactive immune responses and central nervous system inflammation during active experimental autoimmune encephalomyelitis. J Immunol 169, 4712-4716.

Kohm, A.P., McMahon, J.S., Podojil, J.R., Begolka, W.S., DeGutes, M., Kasprowicz, D.J., Ziegler, S.F., and Miller, S.D. (2006). Cutting Edge: anti-CD25 monoclonal antibody injection results in the functional inactivation, not depletion, of CD4+CD25+ T regulatory cells. J Immunol 176, 3301-3305.

Kojima, A., Taguchi, O., and Nishizuka, Y. (1980). Experimental production of possible autoimmune castritis followed by macrocytic anemia in athymic nude mice. Lab Invest 42, 387-395.

Kojima, A., Tanaka-Kojima, Y., Sakakura, T., and Nishizuka, Y. (1976). Prevention of postthymectomy autoimmune thyroiditis in mice. Lab Invest 34, 601-605. 
Korn, T., Reddy, J., Gao, W., Bettelli, E., Awasthi, A., Petersen, T.R., Backstrom, B.T., Sobel, R.A., Wucherpfennig, K.W., Strom, T.B., et al. (2007). Myelin-specific regulatory T-cells accumulate in the CNS but fail to control autoimmune inflammation. Nat Med 13, 423-431.

Kretschmer, K., Apostolou, I., Hawiger, D., Khazaie, K., Nussenzweig, M.C., and von Boehmer, H. (2005). Inducing and expanding regulatory T-cell populations by foreign antigen. Nat Immunol 6, 1219-1227.

Krishnamoorthy, G., Saxena, A., Mars, L.T., Domingues, H.S., Mentele, R., Ben-Nun, A., Lassmann, H., Dornmair, K., Kurschus, F.C., Liblau, R.S., and Wekerle, H. (2009). Myelin-specific T-cells also recognize neuronal autoantigen in a transgenic mouse model of multiple sclerosis. Nat Med 15, 626-632.

Kryczek, I., Wei, S., Zou, L., Zhu, G., Mottram, P., Xu, H., Chen, L., and Zou, W. (2006). Cutting Edge: induction of B7-H4 on APCs through IL-10: novel suppressive mode for regulatory T-cells. J Immunol 177, 40-44.

Kullberg, M.C., Hay, V., Cheever, A.W., Mamura, M., Sher, A., Letterio, J.J., Shevach, E.M., and Piccirillo, C.A. (2005). TGF-beta 1 production by CD4+ CD25+ regulatory Tcells is not essential for suppression of intestinal inflammation. Eur J Immunol 35, 28862895.

Lacorazza, H.D., Tucek-Szabo, C., Vasovic, L.V., Remus, K., and Nikolich-Zugich, J. (2001). Premature TCR alpha beta expression and signaling in early thymocytes impair thymocyte expansion and partially block their development. J Immunol 166, 3184-3193.

Laschinger, M., Vajkoczy, P., and Engelhardt, B. (2002). Encephalitogenic T-cells use LFA-1 for transendothelial migration but not during capture and initial adhesion strengthening in healthy spinal cord microvessels in vivo. Eur J Immunol 32, 3598-3606.

Lathrop, S.K., Santacruz, N.A., Pham, D., Luo, J., and Hsieh, C.S. (2008). Antigenspecific peripheral shaping of the natural regulatory T-cell population. J Exp Med 205, 3105-3117.

Laurence, A., Tato, C.M., Davidson, T.S., Kanno, Y., Chen, Z., Yao, Z., Blank, R.B., Meylan, F., Siegel, R., Hennighausen, L., et al. (2007). Interleukin-2 signaling via STAT5 constrains T helper 17 cell generation. Immunity 26, 371-381.

Lefranc, M.P., Giudicelli, V., Ginestoux, C., Bodmer, J., Muller, W., Bontrop, R., Lemaitre, M., Malik, A., Barbie, V., and Chaume, D. (1999). IMGT, the international ImMunoGeneTics database. Nucleic Acids Res 27, 209-212. 
Liang, B., Workman, C., Lee, J., Chew, C., Dale, B.M., Colonna, L., Flores, M., Li, N., Schweighoffer, E., Greenberg, S., et al. (2008). Regulatory T-cells inhibit dendritic cells by lymphocyte activation gene-3 engagement of MHC class II. J Immunol 180, 59165926.

Liang, S., Alard, P., Zhao, Y., Parnell, S., Clark, S.L., and Kosiewicz, M.M. (2005). Conversion of CD4+ CD25- cells into CD4+ CD25+ regulatory T-cells in vivo requires B7 costimulation, but not the thymus. J Exp Med 201, 127-137.

Lin, M.Y. and Welsh, R.M. (1998). Stability and diversity of T-cell receptor repertoire usage during lymphocytic choriomeningitis virus infection of mice. J Exp Med 188, 1993-2005.

Lio, C.W. and Hsieh, C.S. (2008). A two-step process for thymic regulatory T-cell development. Immunity 28, 100-111.

Liston, A., Lesage, S., Wilson, J., Peltonen, L., and Goodnow, C.C. (2003). Aire regulates negative selection of organ-specific T-cells. Nat Immunol 4, 350-354.

Liston, A., and Rudensky, A.Y. (2007). Thymic development and peripheral homeostasis of regulatory T-cells. Curr Opin Immunol 19, 176-185.

Liu, Y., Teige, I., Birnir, B., and Issazadeh-Navikas, S. (2006). Neuron-mediated generation of regulatory T-cells from encephalitogenic T-cells suppresses EAE. Nat Med $12,518-525$.

Lohr, J., Knoechel, B., Jiang, S., Sharpe, A.H., and Abbas, A.K. (2003). The inhibitory function of B7 costimulators in T-cell responses to foreign and self-antigens. Nat Immunol 4, 664-669.

Lyck, R., Reiss, Y., Gerwin, N., Greenwood, J., Adamson, P., and Engelhardt, B. (2003). T-cell interaction with ICAM-1/ICAM-2 double-deficient brain endothelium in vitro: the cytoplasmic tail of endothelial ICAM-1 is necessary for transendothelial migration of Tcells. Blood 102, 3675-3683.

Madrenas, J., Wange, R.L., Wang, J.L., Isakov, N., Samelson, L.E., and Germain, R.N. (1995). Zeta phosphorylation without ZAP-70 activation induced by TCR antagonists or partial agonists. Science 267, 515-518.

Malek, T.R. and Bayer, A.L. (2004). Tolerance, not immunity, crucially depends on IL-2. Nat Rev Immunol 4, 665-674.

Malek, T.R., Yu, A., Vincek, V., Scibelli, P., and Kong, L. (2002). CD4 regulatory Tcells prevent lethal autoimmunity in IL-2Rbeta-deficient mice. Implications for the nonredundant function of IL-2. Immunity 17, 167-178. 
Malherbe, L., Haus1, C., Teyton, L., and McHeyzer-Williams, M.G. (2004). Clonal selection of helper T-cells is determined by an affinity threshold with no further skewing of TCR binding properties. Immunity 21, 669-679.

Manicassamy, S., Ravindran, R., Deng, J., Oluoch, H., Denning, T.L., Kasturi, S.P., Rosenthal, K.M., Evavold, B.D., and Pulendran, B. (2009). Toll-like receptor 2dependent induction of vitamin A-metabolizing enzymes in dendritic cells promotes $\mathrm{T}$ regulatory responses and inhibits autoimmunity. Nat Med 15, 401-409.

Manolios, N., Letourneur, F., Bonifacino, J.S., and Klausner, R.D. (1991). Pairwise, cooperative and inhibitory interactions describe the assembly and probable structure of the T-cell antigen receptor. EMBO J 10, 1643-1651.

Mason, D. (1998). A very high level of crossreactivity is an essential feature of the T-cell receptor. Immunol Today 19, 395-404.

Maverakis, E., van den Elzen, P., and Sercarz, E.E. (2001). Self-reactive T-cells and degeneracy of T-cell recognition: evolving concepts-from sequence homology to shape mimicry and TCR flexibility. J Autoimmun 16, 201-209.

McGeachy, M.J., Stephens, L.A., and Anderton, S.M. (2005). Natural recovery and protection from autoimmune encephalomyelitis: contribution of CD4+CD25+ regulatory cells within the central nervous system. J Immunol 175, 3025-3032.

McHugh, R.S., Whitters, M.J., Piccirillo, C.A., Young, D.A., Shevach, E.M., Collins, M., and Byrne, M.C. (2002). CD4(+)CD25(+) immunoregulatory T-cells: gene expression analysis reveals a functional role for the glucocorticoid-induced TNF receptor. Immunity $16,311-323$.

McRae, B.L., Vanderlugt, C.L., Dal Canto, M.C., and Miller, S.D. (1995). Functional evidence for epitope spreading in the relapsing pathology of experimental autoimmune encephalomyelitis. J Exp Med 182, 75-85.

Mekala, D.J., Alli, R.S., and Geiger, T.L. (2005). IL-10-dependent infectious tolerance after the treatment of experimental allergic encephalomyelitis with redirected CD4+CD25+ T-lymphocytes. Proc Natl Acad Sci U S A 102, 11817-11822.

Mendel, I., Kerlero de Rosbo, N., and Ben-Nun, A. (1995). A myelin oligodendrocyte glycoprotein peptide induces typical chronic experimental autoimmune encephalomyelitis in $\mathrm{H}-2 \mathrm{~b}$ mice: fine specificity and $\mathrm{T}$-cell receptor $\mathrm{V}$ beta expression of encephalitogenic T-cells. Eur J Immunol 25, 1951-1959. 
Mendel, I., Kerlero de Rosbo, N., and Ben-Nun, A. (1996). Delineation of the minimal encephalitogenic epitope within the immunodominant region of myelin oligodendrocyte glycoprotein: diverse $\mathrm{V}$ beta gene usage by $\mathrm{T}$-cells recognizing the core epitope encephalitogenic for T-cell receptor $\mathrm{V}$ beta $\mathrm{b}$ and $\mathrm{T}$-cell receptor $\mathrm{V}$ beta $\mathrm{a} \mathrm{H}-2 \mathrm{~b}$ mice. Eur J Immunol 26, 2470-2479.

Miller, J.F. and Heath, W.R. (1993). Self-ignorance in the peripheral T-cell pool. Immunol Rev 133, 131-150.

Mombaerts, P., Mizoguchi, E., Grusby, M.J., Glimcher, L.H., Bhan, A.K., and Tonegawa, S. (1993). Spontaneous development of inflammatory bowel disease in T-cell receptor mutant mice. Cell 75, 274-282.

Mucida, D., Park, Y., Kim, G., Turovskaya, O., Scott, I., Kronenberg, M., and Cheroutre, H. (2007). Reciprocal TH17 and regulatory T-cell differentiation mediated by retinoic acid. Science 317, 256-260.

Nakamura, K., Kitani, A., and Strober, W. (2001). Cell contact-dependent immunosuppression by $\mathrm{CD} 4(+) \mathrm{CD} 25(+)$ regulatory T-cells is mediated by cell surfacebound transforming growth factor beta. J Exp Med 194, 629-644.

Ndhlovu, L.C., Takeda, I., Sugamura, K., and Ishii, N. (2004). Expanding role of T-cell costimulators in regulatory T-cell function: recent advances in accessory molecules expressed on both regulatory and nonregulatory T-cells. Crit Rev Immunol 24, 251-266.

Nishizuka, Y. and Sakakura, T. (1969). Thymus and reproduction: sex-linked dysgenesia of the gonad after neonatal thymectomy in mice. Science 166, 753-755.

O'Connor, R.A., Malpass, K.H., and Anderton, S.M. (2007). The inflamed central nervous system drives the activation and rapid proliferation of Foxp3+ regulatory T-cells. J Immunol 179, 958-966.

Offner, H., Vainiene, M., Gold, D.P., Celnik, B., Wang, R., Hashim, G.A., and Vandenbark, A.A. (1992). Characterization of the immune response to a secondary encephalitogenic epitope of basic protein in Lewis rats. I. T-cell receptor peptide regulation of T-cell clones expressing cross-reactive V beta genes. J Immunol 148, 17061711.

Oksenberg, J.R., Panzara, M.A., Begovich, A.B., Mitchell, D., Erlich, H.A., Murray, R.S., Shimonkevitz, R., Sherritt, M., Rothbard, J., and Bernard, C.C. (1993). Selection for Tcell receptor $\mathrm{V}$ beta-D beta-J beta gene rearrangements with specificity for a myelin basic protein peptide in brain lesions of multiple sclerosis. Nature 362, 68-70.

Olivares-Villagomez, D., Wensky, A.K., Wang, Y., and Lafaille, J.J. (2000). Repertoire requirements of CD4+ T-cells that prevent spontaneous autoimmune encephalomyelitis. J Immunol 164, 5499-5507. 
Oosterwegel, M.A., Greenwald, R.J., Mandelbrot, D.A., Lorsbach, R.B., and Sharpe, A.H. (1999). CTLA-4 and T-cell activation. Curr Opin Immunol 11, 294-300.

Oswald-Richter, K., Grill, S.M., Shariat, N., Leelawong, M., Sundrud, M.S., Haas, D.W., and Unutmaz, D. (2004). HIV infection of naturally occurring and genetically reprogrammed human regulatory T-cells. PLoS Biol 2, E198.

Pacholczyk, R., Ignatowicz, H., Kraj, P., and Ignatowicz, L. (2006). Origin and T-cell receptor diversity of Foxp3+CD4+CD25+ T-cells. Immunity 25, 249-259.

Pacholczyk, R., and Kern, J. (2008). The T-cell receptor repertoire of regulatory T-cells. Immunology 125, 450-458.

Pacholczyk, R., Kern, J., Singh, N., Iwashima, M., Kraj, P., and Ignatowicz, L. (2007). Nonself-antigens are the cognate specificities of Foxp3+ regulatory T-cells. Immunity 27, 493-504.

Pacholczyk, R., Kraj, P., and Ignatowicz, L. (2002). Peptide specificity of thymic selection of CD4+CD25+ T-cells. J Immunol 168, 613-620.

Pannetier, C., Even, J., and Kourilsky, P. (1995). T-cell repertoire diversity and clonal expansions in normal and clinical samples. Immunol Today 16, 176-181.

Pantaleo, G., Demarest, J.F., Soudeyns, H., Graziosi, C., Denis, F., Adelsberger, J.W., Borrow, P., Saag, M.S., Shaw, G.M., and Sekaly, R.P., et al. (1994). Major expansion of $\mathrm{CD} 8+\mathrm{T}$-cells with a predominant $\mathrm{V}$ beta usage during the primary immune response to HIV. Nature 370, 463-467.

Papiernik, M., de Moraes, M.L., Pontoux, C., Vasseur, F., and Penit, C. (1998). Regulatory CD4 T-cells: expression of IL-2R alpha chain, resistance to clonal deletion and IL-2 dependency. Int Immunol 10, 371-378.

Paust, S., Lu, L., McCarty, N., and Cantor, H. (2004). Engagement of B7 on effector Tcells by regulatory T-cells prevents autoimmune disease. Proc Natl Acad Sci U S A 101, 10398-10403.

Pennington, D.J., Silva-Santos, B., Silberzahn, T., Escorcio-Correia, M., Woodward, M.J., Roberts, S.J., Smith, A.L., Dyson, P.J., and Hayday, A.C. (2006). Early events in the thymus affect the balance of effector and regulatory T-cells. Nature 444, 1073-1077.

Persons, D.A., Allay, J.A., Allay, E.R., Smeyne, R.J., Ashmun, R.A., Sorrentino, B.P., and Nienhuis, A.W. (1997). Retroviral-mediated transfer of the green fluorescent protein gene into murine hematopoietic cells facilitates scoring and selection of transduced progenitors in vitro and identification of genetically modified cells in vivo. Blood 90, 1777-1786. 
Petersen, T.R., Bettelli, E., Sidney, J., Sette, A., Kuchroo, V., and Backstrom, B.T. (2004). Characterization of MHC- and TCR-binding residues of the myelin oligodendrocyte glycoprotein 38-51 peptide. Eur J Immunol 34, 165-173.

Picca, C.C., Larkin, J., 3rd, Boesteanu, A., Lerman, M.A., Rankin, A.L., and Caton, A.J. (2006). Role of TCR specificity in CD4+ CD25+ regulatory T-cell selection. Immunol Rev 212, 74-85.

Piccirillo, C.A., Letterio, J.J., Thornton, A.M., McHugh, R.S., Mamura, M., Mizuhara, H., and Shevach, E.M. (2002). CD4(+)CD25(+) regulatory T-cells can mediate suppressor function in the absence of transforming growth factor betal production and responsiveness. J Exp Med 196, 237-246.

Piccirillo, C.A. and Shevach, E.M. (2001). Cutting Edge: control of CD8+ T-cell activation by CD4+CD25+ immunoregulatory cells. J Immunol 167, 1137-1140.

Quintana, F.J., Basso, A.S., Iglesias, A.H., Korn, T., Farez, M.F., Bettelli, E., Caccamo, M., Oukka, M., and Weiner, H.L. (2008). Control of T(reg) and T(H)17 cell differentiation by the aryl hydrocarbon receptor. Nature 453, 65-71.

Read, S., Malmstrom, V., and Powrie, F. (2000). Cytotoxic T-lymphocyte-associated antigen 4 plays an essential role in the function of CD25(+)CD4(+) regulatory cells that control intestinal inflammation. J Exp Med 192, 295-302.

Razzaq, T.M., Ozegbe, P., Jury, E.C., Sembi, P., Blackwell, N.M., and Kabouridis, P.S. (2004). Regulation of T-cell receptor signalling by membrane microdomains. Immunology 113, 413-426.

Reddy, J., Illes, Z., Zhang, X., Encinas, J., Pyrdol, J., Nicholson, L., Sobel, R.A., Wucherpfennig, K.W., and Kuchroo, V.K. (2004). Myelin proteolipid protein-specific $\mathrm{CD} 4+\mathrm{CD} 25+$ regulatory cells mediate genetic resistance to experimental autoimmune encephalomyelitis. Proc Natl Acad Sci U S A 101, 15434-15439.

Reddy, J., Waldner, H., Zhang, X., Illes, Z., Wucherpfennig, K.W., Sobel, R.A., and Kuchroo, V.K. (2005). Cutting Edge: CD4+CD25+ regulatory T-cells contribute to gender differences in susceptibility to experimental autoimmune encephalomyelitis. J Immunol 175, 5591-5595.

Romagnoli, P., Hudrisier, D., and van Meerwijk, J.P. (2002). Preferential recognition of self antigens despite normal thymic deletion of $\mathrm{CD} 4(+) \mathrm{CD} 25(+)$ regulatory T-cells. J Immunol 168, 1644-1648.

Rubtsov, Y.P., Rasmussen, J.P., Chi, E.Y., Fontenot, J., Castelli, L., Ye, X., Treuting, P., Siewe, L., Roers, A., Henderson, W.R., Jr., et al. (2008). Regulatory T-cell-derived interleukin-10 limits inflammation at environmental interfaces. Immunity 28, 546-558. 
Sakaguchi, S., Sakaguchi, N., Asano, M., Itoh, M., and Toda, M. (1995). Immunologic self-tolerance maintained by activated T-cells expressing IL-2 receptor alpha-chains (CD25). Breakdown of a single mechanism of self-tolerance causes various autoimmune diseases. J Immunol 155, 1151-1164.

Sakaguchi, S., Takahashi, T., and Nishizuka, Y. (1982). Study on cellular events in postthymectomy autoimmune oophoritis in mice. II. Requirement of Lyt-1 cells in normal female mice for the prevention of oophoritis. J Exp Med 156, 1577-1586.

Salomon, B., Lenschow, D.J., Rhee, L., Ashourian, N., Singh, B., Sharpe, A., and Bluestone, J.A. (2000). B7/CD28 costimulation is essential for the homeostasis of the CD4+CD25+ immunoregulatory T-cells that control autoimmune diabetes. Immunity 12 , 431-440.

Schimpl, A. and Wecker, E. (1972). Replacement of T-cell function by a T-cell product. Nat New Biol 237, 15-17.

Schmedt, C. and Tarakhovsky, A. (2001). Autonomous maturation of alpha/beta T lineage cells in the absence of COOH-terminal Src kinase (Csk). J Exp Med 193, 815-826.

Schwartz, R.H. (1990). A cell culture model for T-lymphocyte clonal anergy. Science 248, 1349-1356.

Selvaraj, R.K. and Geiger, T.L. (2007). A kinetic and dynamic analysis of Foxp3 induced in T-cells by TGF-beta. J Immunol 178, 7667-7677.

Selvaraj, R.K. and Geiger, T.L. (2008). Mitigation of experimental allergic encephalomyelitis by TGF-beta induced Foxp3+ regulatory T-lymphocytes through the induction of anergy and infectious tolerance. J Immunol 180, 2830-2838.

Serra, P., Amrani, A., Yamanouchi, J., Han, B., Thiessen, S., Utsugi, T., Verdaguer, J., and Santamaria, P. (2003). CD40 ligation releases immature dendritic cells from the control of regulatory CD4+CD25+ T-cells. Immunity 19, 877-889.

Shendure, J. and Ji, H. (2008). Next-generation DNA sequencing. Nat Biotechnol 26, $1135-1145$.

Shimizu, J., Yamazaki, S., Takahashi, T., Ishida, Y., and Sakaguchi, S. (2002).

Stimulation of CD25(+)CD4(+) regulatory T-cells through GITR breaks immunological self-tolerance. Nat Immunol 3, 135-142.

Sobel, R.A. and Kuchroo, V.K. (1992). The immunopathology of acute experimental allergic encephalomyelitis induced with myelin proteolipid protein. T-cell receptors in inflammatory lesions. J Immunol 149, 1444-1451. 
Sourdive, D.J., Murali-Krishna, K., Altman, J.D., Zajac, A.J., Whitmire, J.K., Pannetier, C., Kourilsky, P., Evavold, B., Sette, A., and Ahmed, R. (1998). Conserved T-cell receptor repertoire in primary and memory CD8 T-cell responses to an acute viral infection. J Exp Med 188, 71-82.

Starr, T.K., Jameson, S.C., and Hogquist, K.A. (2003). Positive and negative selection of T-cells. Annu Rev Immunol 21, 139-176.

Steffen, B.J., Butcher, E.C., and Engelhardt, B. (1994). Evidence for involvement of ICAM-1 and VCAM-1 in lymphocyte interaction with endothelium in experimental autoimmune encephalomyelitis in the central nervous system in the SJL/J mouse. Am J Pathol 145, 189-201.

Stephens, L.A., Gray, D., and Anderton, S.M. (2005). CD4+CD25+ regulatory T-cells limit the risk of autoimmune disease arising from T-cell receptor crossreactivity. Proc Natl Acad Sci U S A 102, 17418-17423.

Sun, C.M., Hall, J.A., Blank, R.B., Bouladoux, N., Oukka, M., Mora, J.R., and Belkaid, Y. (2007). Small intestine lamina propria dendritic cells promote de novo generation of Foxp3 T reg cells via retinoic acid. J Exp Med 204, 1775-1785.

Suri-Payer, E., Amar, A.Z., Thornton, A.M., and Shevach, E.M. (1998). CD4+CD25+ Tcells inhibit both the induction and effector function of autoreactive T-cells and represent a unique lineage of immunoregulatory cells. J Immunol 160, 1212-1218.

Tai, X., Cowan, M., Feigenbaum, L., and Singer, A. (2005). CD28 costimulation of developing thymocytes induces Foxp3 expression and regulatory T-cell differentiation independently of interleukin 2. Nat Immunol 6, 152-162.

Takahashi, T., Kuniyasu, Y., Toda, M., Sakaguchi, N., Itoh, M., Iwata, M., Shimizu, J., and Sakaguchi, S. (1998). Immunologic self-tolerance maintained by CD25+CD4+ naturally anergic and suppressive T-cells: induction of autoimmune disease by breaking their anergic/suppressive state. Int Immunol 10, 1969-1980.

Takahashi, T., Tagami, T., Yamazaki, S., Uede, T., Shimizu, J., Sakaguchi, N., Mak, T.W., and Sakaguchi, S. (2000). Immunologic self-tolerance maintained by $\mathrm{CD} 25(+) \mathrm{CD} 4(+)$ regulatory T-cells constitutively expressing cytotoxic T-lymphocyteassociated antigen 4. J Exp Med 192, 303-310.

Tang, Q. and Bluestone, J.A. (2006). Regulatory T-cell physiology and application to treat autoimmunity. Immunol Rev 212, 217-237.

Tang, Q., Henriksen, K.J., Bi, M., Finger, E.B., Szot, G., Ye, J., Masteller, E.L., McDevitt, H., Bonyhadi, M., and Bluestone, J.A. (2004). In vitro-expanded antigenspecific regulatory T-cells suppress autoimmune diabetes. J Exp Med 199, 1455-1465. 
Thornton, A.M., Piccirillo, C.A., and Shevach, E.M. (2004). Activation requirements for the induction of CD4+CD25+ T-cell suppressor function. Eur J Immunol 34, 366-376.

Thornton, A.M. and Shevach, E.M. (1998). CD4+CD25+ immunoregulatory T-cells suppress polyclonal $\mathrm{T}$-cell activation in vitro by inhibiting interleukin 2 production. J Exp Med 188, 287-296.

Thornton, A.M. and Shevach, E.M. (2000). Suppressor effector function of CD4+CD25+ immunoregulatory T-cells is antigen nonspecific. J Immunol 164, 183-190.

Torres-Nagel, N., Deutschlander, A., Herrmann, T., Arden, B., and Hunig, T. (1997). Control of TCR V alpha-mediated positive repertoire selection and alloreactivity by differential J alpha usage and CDR3 alpha composition. Int Immunol 9, 1441-1452.

Trautmann, L., Rimbert, M., Echasserieau, K., Saulquin, X., Neveu, B., Dechanet, J., Cerundolo, V., and Bonneville, M. (2005). Selection of T-cell clones expressing highaffinity public TCRs within Human cytomegalovirus-specific CD8 T-cell responses. J Immunol 175, 6123-6132.

Turner, S.J., Doherty, P.C., McCluskey, J., and Rossjohn, J. (2006). Structural determinants of T-cell receptor bias in immunity. Nat Rev Immunol 6, 883-894.

Udyavar, A., Alli, R., Nguyen, P., Baker, L., and Geiger, T.L. (2009). Subtle affinityenhancing mutations in a myelin oligodendrocyte glycoprotein-specific TCR alter specificity and generate new self-reactivity. J Immunol 182, 4439-4447.

van Santen, H.M., Benoist, C., and Mathis, D. (2004). Number of T reg cells that differentiate does not increase upon encounter of agonist ligand on thymic epithelial cells. J Exp Med 200, 1221-1230.

Vanderlugt, C.L., and Miller, S.D. (2002). Epitope spreading in immune-mediated diseases: implications for immunotherapy. Nat Rev Immunol 2, 85-95.

Vanderlugt, C.L., Neville, K.L., Nikcevich, K.M., Eagar, T.N., Bluestone, J.A., and Miller, S.D. (2000). Pathologic role and temporal appearance of newly emerging autoepitopes in relapsing experimental autoimmune encephalomyelitis. J Immunol 164, 670-678.

Veldhoen, M., Hocking, R.J., Atkins, C.J., Locksley, R.M., and Stockinger, B. (2006). TGFbeta in the context of an inflammatory cytokine milieu supports de novo differentiation of IL-17-producing T-cells. Immunity 24, 179-189.

Vignali, D.A., Collison, L.W., and Workman, C.J. (2008). How regulatory T-cells work. Nat Rev Immunol 8, 523-532. 
Walker, L.S., Chodos, A., Eggena, M., Dooms, H., and Abbas, A.K. (2003). Antigendependent proliferation of CD4+ CD25+ regulatory T-cells in vivo. J Exp Med 198, 249258.

Wan, Y.Y. and Flavell, R.A. (2007). Regulatory T-cell functions are subverted and converted owing to attenuated Foxp3 expression. Nature 445, 766-770.

Wang, J., Huizinga, T.W., and Toes, R.E. (2009). De novo generation and enhanced suppression of human CD4+CD25+ regulatory T-cells by retinoic acid. J Immunol 183, 4119-4126.

Whitham, R.H., Bourdette, D.N., Hashim, G.A., Herndon, R.M., Ilg, R.C., Vandenbark, A.A., and Offner, H. (1991). Lymphocytes from SJL/J mice immunized with spinal cord respond selectively to a peptide of proteolipid protein and transfer relapsing demyelinating experimental autoimmune encephalomyelitis. J Immunol 146, 101-107.

Wilson, D.B., Wilson, D.H., Schroder, K., Pinilla, C., Blondelle, S., Houghten, R.A., and Garcia, K.C. (2004). Specificity and degeneracy of T-cells. Mol Immunol 40, 1047-1055.

Wong, J., Mathis, D., and Benoist, C. (2007a). TCR-based lineage tracing: no evidence for conversion of conventional into regulatory $\mathrm{T}$-cells in response to a natural self-antigen in pancreatic islets. J Exp Med 204, 2039-2045.

Wong, J., Obst, R., Correia-Neves, M., Losyev, G., Mathis, D., and Benoist, C. (2007b). Adaptation of TCR repertoires to self-peptides in regulatory and nonregulatory CD4+ Tcells. J Immunol 178, 7032-7041.

Workman, C.J., Szymczak-Workman, A.L., Collison, L.W., Pillai, M.R., and Vignali, D.A. (2009). The development and function of regulatory T-cells. Cell Mol Life Sci 66, 2603-2622.

Workman, C.J., and Vignali, D.A. (2005). Negative regulation of T-cell homeostasis by lymphocyte activation gene-3 (CD223). J Immunol 174, 688-695.

Wucherpfennig, K.W., and Strominger, J.L. (1995). Molecular mimicry in T-cellmediated autoimmunity: viral peptides activate human T-cell clones specific for myelin basic protein. Cell 80,695-705.

Yagi, H., Nomura, T., Nakamura, K., Yamazaki, S., Kitawaki, T., Hori, S., Maeda, M., Onodera, M., Uchiyama, T., Fujii, S., and Sakaguchi, S. (2004). Crucial role of FoxP3 in the development and function of human CD25+CD4+ regulatory T-cells. Int Immunol 16, 1643-1656.

Yoshizawa, I., Bronson, R., Ben-Nun, A., Richert, J.R., Dorf, M.E., and AbromsonLeeman, S. (1998). Differential recognition of MBP epitopes in BALB/c mice determines the site of inflammatory disease induction. J Neuroimmunol 89, 73-82. 
You, S., Leforban, B., Garcia, C., Bach, J.F., Bluestone, J.A., and Chatenoud, L. (2007). Adaptive TGF-beta-dependent regulatory T-cells control autoimmune diabetes and are a privileged target of anti-CD3 antibody treatment. Proc Natl Acad Sci U S A 104, 63356340 .

Yu, P., Gregg, R.K., Bell, J.J., Ellis, J.S., Divekar, R., Lee, H.H., Jain, R., Waldner, H., Hardaway, J.C., Collins, M., et al. (2005). Specific T regulatory cells display broad suppressive functions against experimental allergic encephalomyelitis upon activation with cognate antigen. J Immunol 174, 6772-6780.

Zamvil, S., Nelson, P., Trotter, J., Mitchell, D., Knobler, R., Fritz, R., and Steinman, L. (1985). T-cell clones specific for myelin basic protein induce chronic relapsing paralysis and demyelination. Nature 317, 355-358.

Zamvil, S.S., and Steinman, L. (1990). The T-lymphocyte in experimental allergic encephalomyelitis. Annu Rev Immunol 8, 579-621.

Zehn, D., and Bevan, M.J. (2006). T-cells with low avidity for a tissue-restricted antigen routinely evade central and peripheral tolerance and cause autoimmunity. Immunity 25, 261-270.

Zhang, X., Koldzic, D.N., Izikson, L., Reddy, J., Nazareno, R.F., Sakaguchi, S., Kuchroo, V.K., and Weiner, H.L. (2004). IL-10 is involved in the suppression of experimental autoimmune encephalomyelitis by CD25+CD4+ regulatory T-cells. Int Immunol 16, 249256.

Zhao, D.M., Thornton, A.M., DiPaolo, R.J., and Shevach, E.M. (2006). Activated CD4+CD25+ T-cells selectively kill B lymphocytes. Blood 107, 3925-3932.

Zwar, T.D., van Driel, I.R., and Gleeson, P.A. (2006). Guarding the immune system: suppression of autoimmunity by CD4+CD25+ immunoregulatory T-cells. Immunol Cell Biol 84, 487-501. 


\section{VITA}

Xin Liu was born in Heilongjiang Province at P.R.China in 1979. In 1997, she attended Shanghai Medical University (Merged with Fudan University in 1999), major in Clinical Medicine. After achieving her Bachelor of Medicine in 2002, she joined residency program of OBGYN in International Peace Maternity and Child Health Hospital. In August 2005, she chose to enter the Integrated Program in Biomedical Sciences at the University of Tennessee Health Science Center for Ph.D. studies. In May 2006, she joined Dr. Terrence L. Geiger's laboratory at St. Jude Children's Research Hospital and conducted research in the repertoire analysis of regulatory T-cells in EAE. She received her Doctor of Philosophy degree from University of Tennessee in May of 2010. 